Tom 24 № 3

\title{
СТАБИЛИЗАТОРЫ ВЕРШИН ГРАФОВ С ПРИМИТИВНЫМИ ГРУППАМИ АВТОМОРФИЗМОВ И УСИЛЕННАЯ ВЕРСИЯ ГИПОТЕЗЫ СИМСА. IV ${ }^{1}$
}

\author{
А. С. Кондратьев, В. И. Трофимов
}

\begin{abstract}
Четвертая из цикла статей, результаты которого влекут справедливость усиленной версии гипотезы Симса о конечных примитивных группах подстановок. Данная статья посвящена рассмотрению случая примитивных групп подстановок с простым цоколем ортогонального лиева типа и непараболическим стабилизатором точки. Пусть $G$ - конечная группа и $M_{1}, M_{2}$ - различные сопряженные максимальные подгруппы группы $G$. Для каждого $i \in \mathbb{N}$ индуктивно определим подгруппы $\left(M_{1}, M_{2}\right)^{i}$ и $\left(M_{2}, M_{1}\right)^{i}$ из $M_{1} \cap M_{2}$, называемые нами $i$-ми взаимными ядрами подгруппы $M_{1}$ относительно $M_{2}$ и подгруппы $M_{2}$ относительно $M_{1}$ соответственно. Положим $\left(M_{1}, M_{2}\right)^{1}=\left(M_{1} \cap M_{2}\right)_{M_{1}}$ и $\left(M_{2}, M_{1}\right)^{1}=\left(M_{1} \cap M_{2}\right)_{M_{2}}$. Для $i \in \mathbb{N}$, предполагая, что $\left(M_{1}, M_{2}\right)^{i}$ и $\left(M_{2}, M_{1}\right)^{i}$ уже определены, положим $\left(M_{1}, M_{2}\right)^{i+1}=\left(\left(M_{1}, M_{2}\right)^{i} \cap\left(M_{2}, M_{1}\right)^{i}\right)_{M_{1}}$ и $\left(M_{2}, M_{1}\right)^{i+1}=\left(\left(M_{1}, M_{2}\right)^{i} \cap\left(M_{2}, M_{1}\right)^{i}\right)_{M_{2}}$. Нас интересует случай, когда $\left(M_{1}\right)_{G}=\left(M_{2}\right)_{G}=1$ и $1<\left|\left(M_{1}, M_{2}\right)^{2}\right| \leq\left|\left(M_{2}, M_{1}\right)^{2}\right|$. Множество всех таких троек $\left(G, M_{1}, M_{2}\right)$ обозначается через П. Мы рассматриваем тройки из П с точностью до следующей эквивалентности: тройки $\left(G, M_{1}, M_{2}\right)$ и $\left(G^{\prime}, M_{1}^{\prime}, M_{2}^{\prime}\right)$ из П эквивалентны, если существует изоморфизм $G$ на $G^{\prime}$, отображающий $M_{1}$ на $M_{1}^{\prime}$ и $M_{2}$ на $M_{2}^{\prime}$.
\end{abstract}

В данной статье доказана следующая теорема.

Теорема. Пусть $\left(G, M_{1}, M_{2}\right) \in \Pi, L=\operatorname{Soc}(G)$ - простая ортогональная группа степени $\geq 7 u$ $M_{1} \cap L-$ непараболическал подгруппа в $L$. Тогда $L \cong O_{8}^{+}(r)$, где $r$ - некоторое нечетное простое число, $\left(M_{1}, M_{2}\right)^{3}=\left(M_{2}, M_{1}\right)^{3}=1$ и выполняется одно из следующих утверждений:

(a) $r \equiv \pm 1(\bmod 8)$, группа $G$ изоморфна $O_{8}^{+}(r): \mathbb{Z}_{3}$ или $O_{8}^{+}(r): S_{3},\left(M_{1}, M_{2}\right)^{2}=Z\left(O_{2}\left(M_{1}\right)\right) u$ $\left(M_{2}, M_{1}\right)^{2}=Z\left(O_{2}\left(M_{2}\right)\right)$ - элементарные абелевы группы порлдка $2^{3},\left(M_{1}, M_{2}\right)^{1}=O_{2}\left(M_{1}\right) u\left(M_{2}, M_{1}\right)^{1}=$ $\mathrm{O}_{2}\left(M_{2}\right)$ - специальные группы порядка $2^{9}$, группа $M_{1} / O_{2}\left(M_{1}\right)$ изоморфна $L_{3}(2) \times \mathbb{Z}_{3}$ или $L_{3}(2) \times S_{3}$ соответственно и $M_{1} \cap M_{2}$ - силовская 2-подгруппа в $M_{1}$;

(b) $r \leq 5$, группа $G / L$ либо содержит $\operatorname{Outdiag}(L)$, либо изоморфна $\mathbb{Z}_{4},\left(M_{1}, M_{2}\right)^{2}=$ $Z\left(O_{2}\left(M_{1} \cap L\right)\right)$ u $\left(M_{2}, M_{1}\right)^{2}=Z\left(O_{2}\left(M_{2} \cap L\right)\right)$ - элементарные абелевы группы порядка $2^{2}$, $\left(M_{1}, M_{2}\right)^{1}=\left[O_{2}\left(M_{1} \cap L\right), O_{2}\left(M_{1} \cap L\right)\right] u\left(M_{2}, M_{1}\right)^{1}=\left[O_{2}\left(M_{2} \cap L\right), O_{2}\left(M_{2} \cap L\right)\right]-$ элементарные абелевы группы порядка $2^{5}, \mathrm{O}_{2}\left(M_{1} \cap L\right) /\left[\mathrm{O}_{2}\left(M_{1} \cap L\right), O_{2}\left(M_{1} \cap L\right)\right]$ - элементарная абелева группа порлдка $2^{6}$, группа $\left(M_{1} \cap L\right) / O_{2}\left(M_{1} \cap L\right)$ изоморфна $S_{3},\left|M_{1}: M_{1} \cap M_{2}\right|=24,\left|M_{1} \cap M_{2} \cap L\right|=2^{11}$ и элемент порлдка 3 из $M_{1} \cap M_{2}\left(\right.$ при $G / L \cong A_{4}$ или $\left.G / L \cong S_{4}\right)$ индуцирует на группе $L$ ее стандартный графовый автоморфизм.

В каждом из случаев (a) и (b) тройки $\left(G, M_{1}, M_{2}\right)$ существуют и образуют один класс эквивалентноcmu.

Ключевые слова: конечная примитивная группа подстановок, стабилизатор точки, гипотеза Симса, почти простая группа, группа ортогонального лиева типа.

A. S. Kondrat'ev, V. I. Trofimov. Stabilizers of vertices of graphs with primitive automorphism groups and a strong version of the Sims conjecture. IV.

This is the fourth in a series of papers whose results imply the validity of a strengthened version of the Sims conjecture on finite primitive permutation groups. In this paper, the case of primitive groups with simple socle of orthogonal Lie type and nonparabolic point stabilizer is considered. Let $G$ be a finite group, and let $M_{1}$ and $M_{2}$ be distinct conjugate maximal subgroups of $G$. For any $i \in \mathbb{N}$, we define inductively subgroups $\left(M_{1}, M_{2}\right)^{i}$ and $\left(M_{2}, M_{1}\right)^{i}$ of $M_{1} \cap M_{2}$, which will be called the $i$ th mutual cores of $M_{1}$ with respect to $M_{2}$ and of $M_{2}$ with respect to $M_{1}$, respectively. Put $\left(M_{1}, M_{2}\right)^{1}=\left(M_{1} \cap M_{2}\right)_{M_{1}}$ and $\left(M_{2}, M_{1}\right)^{1}=\left(M_{1} \cap M_{2}\right)_{M_{2}}$. For $i \in \mathbb{N}$, assuming that $\left(M_{1}, M_{2}\right)^{i}$ and $\left(M_{2}, M_{1}\right)^{i}$ are already defined, put $\left(M_{1}, M_{2}\right)^{i+1}=\left(\left(M_{1}, M_{2}\right)^{i} \cap\left(M_{2}, M_{1}\right)^{i}\right)_{M_{1}}$ and $\left(M_{2}, M_{1}\right)^{i+1}=\left(\left(M_{1}, M_{2}\right)^{i} \cap\left(M_{2}, M_{1}\right)^{i}\right)_{M_{2}}$. We are interested in the case when $\left(M_{1}\right)_{G}=\left(M_{2}\right)_{G}=1$ and $1<\left|\left(M_{1}, M_{2}\right)^{2}\right| \leq\left|\left(M_{2}, M_{1}\right)^{2}\right|$. The set of all such triples $\left(G, M_{1}, M_{2}\right)$ is denoted by $\Pi$. We consider triples from $\Pi$ up to the following equivalence: triples $\left(G, M_{1}, M_{2}\right)$ and $\left(G^{\prime}, M_{1}^{\prime}, M_{2}^{\prime}\right)$ from $\Pi$ are equivalent if there exists an isomorphism from $G$ to $G^{\prime}$ mapping $M_{1}$ to $M_{1}^{\prime}$ and $M_{2}$ to $M_{2}^{\prime}$. In the present paper, the following theorem is proved.

\footnotetext{
${ }^{1}$ Работа выполнена за счет гранта Российского научного фонда (проект 14-11-00061-П).
} 
Theorem. Suppose that $\left(G, M_{1}, M_{2}\right) \in \Pi, L=\operatorname{Soc}(G)$ is a simple orthogonal group of degree $\geq 7$, and $M_{1} \cap L$ is a nonparabolic subgroup of $L$. Then $L \cong O_{8}^{+}(r)$, where $r$ is an odd prime, $\left(M_{1}, M_{2}\right)^{3}=\left(M_{2}, M_{1}\right)^{3}=1$, and one of the following statements holds:

(a) $r \equiv \pm 1(\bmod 8)$, the group $G$ is isomorphic to $O_{8}^{+}(r): \mathbb{Z}_{3}$ or $O_{8}^{+}(r): S_{3},\left(M_{1}, M_{2}\right)^{2}=Z\left(O_{2}\left(M_{1}\right)\right)$ and $\left(M_{2}, M_{1}\right)^{2}=Z\left(O_{2}\left(M_{2}\right)\right)$ are elementary abelian groups of order $2^{3},\left(M_{1}, M_{2}\right)^{1}=O_{2}\left(M_{1}\right)$ and $\left(M_{2}, M_{1}\right)^{1}=$ $\mathrm{O}_{2}\left(M_{2}\right)$ are special groups of order $2^{9}$, the group $M_{1} / O_{2}\left(M_{1}\right)$ is isomorphic to $L_{3}(2) \times \mathbb{Z}_{3}$ or $L_{3}(2) \times S_{3}$, respectively, and $M_{1} \cap M_{2}$ is a Sylow 2-subgroup in $M_{1}$;

(b) $r \leq 5 ; G / L$ either contains Outdiag $(L)$ or is isomorphic to $\mathbb{Z}_{4}, \quad\left(M_{1}, M_{2}\right)^{2}=$ $Z\left(\mathrm{O}_{2}\left(M_{1} \cap L\right)\right)$ and $\left(M_{2}, M_{1}\right)^{2}=Z\left(O_{2}\left(M_{2} \cap L\right)\right)$ are elementary abelian groups of order $2^{2}$, $\left(M_{1}, M_{2}\right)^{1}=\left[O_{2}\left(M_{1} \cap L\right), O_{2}\left(M_{1} \cap L\right)\right]$ and $\left(M_{2}, M_{1}\right)^{1}=\left[O_{2}\left(M_{2} \cap L\right), O_{2}\left(M_{2} \cap L\right)\right]$ are elementary abelian groups of order $2^{5}, \mathrm{O}_{2}\left(M_{1} \cap L\right) /\left[\mathrm{O}_{2}\left(M_{1} \cap L\right), \mathrm{O}_{2}\left(M_{1} \cap L\right)\right]$ is an elementary abelian group of order $2^{6}$; the group $\left(M_{1} \cap L\right) / O_{2}\left(M_{1} \cap L\right)$ is isomorphic to the group $S_{3},\left|M_{1}: M_{1} \cap M_{2}\right|=24,\left|M_{1} \cap M_{2} \cap L\right|=2^{11}$, and an element of order 3 from $M_{1} \cap M_{2}$ (in the case $G / L \cong A_{4}$ or $G / L \cong S_{4}$ ) induces on the group $L$ its standard graph automorphism.

In any of cases (a) and (b), the triples $\left(G, M_{1}, M_{2}\right)$ from $\Pi$ exist and form one class up to equivalence.

Keywords: finite primitive permutation group, stabilizer of a point, Sims conjecture, almost simple group, group of orthogonal Lie type.

MSC: 20B15, 20D06, 05C25

DOI: $10.21538 / 0134-4889-2018-24-3-109-132$

\section{Введение}

Пусть $G$ - группа и $M_{1}, M_{2}$ - подгруппы группы $G$. Следуя [3], для каждого $i \in \mathbb{N}$ индуктивно определим подгруппы $\left(M_{1}, M_{2}\right)^{i}$ и $\left(M_{2}, M_{1}\right)^{i}$ из $M_{1} \cap M_{2}$, называемые нами $i$-ми взаимными ядрами подгруппы $M_{1}$ относительно $M_{2}$ и подгруппы $M_{2}$ относительно $M_{1}$ соответственно. Положим

$$
\left(M_{1}, M_{2}\right)^{1}=\left(M_{1} \cap M_{2}\right)_{M_{1}},\left(M_{2}, M_{1}\right)^{1}=\left(M_{1} \cap M_{2}\right)_{M_{2}} .
$$

Для $i \in \mathbb{N}$, предполагая, что $\left(M_{1}, M_{2}\right)^{i}$ и $\left(M_{2}, M_{1}\right)^{i}$ уже определены, положим

$$
\left(M_{1}, M_{2}\right)^{i+1}=\left(\left(M_{1}, M_{2}\right)^{i} \cap\left(M_{2}, M_{1}\right)^{i}\right)_{M_{1}},\left(M_{2}, M_{1}\right)^{i+1}=\left(\left(M_{1}, M_{2}\right)^{i} \cap\left(M_{2}, M_{1}\right)^{i}\right)_{M_{2}} .
$$

Нас интересует случай, когда $G$ - конечная группа, $M_{1}$ и $M_{2}$ - различные сопряженные максимальные подгруппы группы $G,\left(M_{1}\right)_{G}=\left(M_{2}\right)_{G}=1$ и $1<\left|\left(M_{1}, M_{2}\right)^{2}\right| \leq\left|\left(M_{2}, M_{1}\right)^{2}\right|$ (соответствующую мотивировку, связанную с усиленной версией гипотезы Симса, см. в $[4$, ч. I]). Множество всех таких троек $\left(G, M_{1}, M_{2}\right)$ обозначается через П. Мы рассматриваем тройки из П с точностью до следующей эквивалентности: тройки $\left(G, M_{1}, M_{2}\right)$ и $\left(G^{\prime}, M_{1}^{\prime}, M_{2}^{\prime}\right)$ из П эквивалентны, если существует изоморфизм $G$ на $G^{\prime}$, отображающий $M_{1}$ на $M_{1}^{\prime}$ и $M_{2}$ на $M_{2}^{\prime}$. Отметим, что согласно [4, ч. I, предложение 1.1(a)] вторые взаимные ядра $\left(M_{1}, M_{2}\right)^{2}$ и $\left(M_{2}, M_{1}\right)^{2}$ являются $p$-группами для некоторого простого числа $p$.

Цель нашего цикла статей - описать множество П. Это описание существенно усилит результаты из [3]. Ранее нами рассмотрены случаи, когда группа $G$ не является почти простой группой или имеет простой знакопеременный цоколь и когда $G$ - группа с простым цоколем $\operatorname{Soc}(G)$ неортогонального лиева типа и $M_{1} \cap \operatorname{Soc}(G)$ - непараболическая подгруппа в $\operatorname{Soc}(G)$ (см. [4]). Данная статья цикла посвящена рассмотрению случая, когда $G$ - группа с простым ортогональным цоколем $\operatorname{Soc}(G)$ и $M_{1} \cap \operatorname{Soc}(G)$ - непараболическая подгруппа в $\operatorname{Soc}(G)$.

Доказана следующая

Теорема. Пусть $\left(G, M_{1}, M_{2}\right) \in \Pi, L=\operatorname{Soc}(G)$ - простая ортогональная группа степени $\geq 7$ и $M_{1} \cap L-$ непараболическая подгруппа в $L$. Тогда $L \cong O_{8}^{+}(r)$, где $r$ - некоторое нечетное простое число, $\left(M_{1}, M_{2}\right)^{3}=\left(M_{2}, M_{1}\right)^{3}=1$ и выполняется одно из следующих утверждений:

(a) $r \equiv \pm 1(\bmod 8)$, группа $G$ изоморфна $O_{8}^{+}(r): \mathbb{Z}_{3}$ или $O_{8}^{+}(r): S_{3},\left(M_{1}, M_{2}\right)^{2}=Z\left(O_{2}\left(M_{1}\right)\right)$ u $\left(M_{2}, M_{1}\right)^{2}=Z\left(O_{2}\left(M_{2}\right)\right)$ - элементарные абелевы группы порядка $2^{3},\left(M_{1}, M_{2}\right)^{1}=O_{2}\left(M_{1}\right)$ 
и $\left(M_{2}, M_{1}\right)^{1}=\mathrm{O}_{2}\left(M_{2}\right)$ - специальные группь порядка $2^{9}$, группа $M_{1} / O_{2}\left(M_{1}\right)$ изоморбна $L_{3}(2) \times \mathbb{Z}_{3}$ или $L_{3}(2) \times S_{3}$ соответственно и $M_{1} \cap M_{2}$ - силовскал 2-подгруппа в $M_{1}$;

(b) $r \leq 5$, группа $G / L$ либо содержит Outdiag $(L)$, либо изоморфна $\mathbb{Z}_{4}$, $\left(M_{1}, M_{2}\right)^{2}=$ $Z\left(O_{2}\left(M_{1} \cap L\right)\right)$ и $\left(M_{2}, M_{1}\right)^{2}=Z\left(O_{2}\left(M_{2} \cap L\right)\right)$ - элементарные абелевы группы порлдка $2^{2}$, $\left(M_{1}, M_{2}\right)^{1}=\left[O_{2}\left(M_{1} \cap L\right), O_{2}\left(M_{1} \cap L\right)\right] u\left(M_{2}, M_{1}\right)^{1}=\left[O_{2}\left(M_{2} \cap L\right), O_{2}\left(M_{2} \cap L\right)\right]$ - элементарные абелевы группы порядка $2^{5}, O_{2}\left(M_{1} \cap L\right) /\left[O_{2}\left(M_{1} \cap L\right), O_{2}\left(M_{1} \cap L\right)\right]$ - элементарная абелева группа порлдка $2^{6}$, группа $\left(M_{1} \cap L\right) / O_{2}\left(M_{1} \cap L\right)$ изоморфна $S_{3},\left|M_{1}: M_{1} \cap M_{2}\right|=24,\left|M_{1} \cap M_{2} \cap L\right|=2^{11}$ и элемент порлдка 3 из $M_{1} \cap M_{2}\left(\right.$ при $G / L \cong A_{4}$ или $\left.G / L \cong S_{4}\right)$ индуцирует на группе $L$ ее стандартный графовый автоморфизм.

В каждом из случаев (a) и (b) тройки $\left(G, M_{1}, M_{2}\right)$ существуют и образуют один класс эквивалентности.

В конце статьи указаны опечатки в [4, ч. III].

\section{1. Обозначения и предварительные результаты}

Используемые в статье терминология и обозначения в основном стандартны (см., например, $[6 ; 8 ; 9 ; 12 ; 13])$. Следуя [6], простая ортогональная группа степени $n$ над полем $G F(q)$ обозначается через $O_{n}(q)$ для нечетного $n$ и через $O_{n}^{\varepsilon}(q)$ для четного $n$, где $\varepsilon \in\{+,-\}$.

В доказательстве теоремы мы используем следующее общее утверждение о взаимных ядрах подгрупп.

Лемма 1.1. Пусть $G$ - конечная группа и $L-$ нормальная подгруппа в $G$. Тогда для произвольных подгрупп $M_{1}, M_{2}$ группы $G$ и произвольного целого положительного числа $i$ имеем

$$
\left(M_{1}, M_{2}\right)^{i} \cap L \leq\left(M_{1} \cap L, M_{2} \cap L\right)^{i} .
$$

Д о к а з а т е л ь с т в о. Поскольку

$$
\begin{gathered}
\left(M_{1}, M_{2}\right)^{1} \cap L=\left(\cap_{g \in M_{1}}\left(\left(M_{1} \cap M_{2}\right)^{g}\right)\right) \cap L \leq\left(\cap_{g \in M_{1} \cap L}\left(\left(M_{1} \cap M_{2}\right)^{g}\right)\right) \cap L \\
=\cap_{g \in M_{1} \cap L}\left(\left(M_{1} \cap M_{2} \cap L\right)^{g}\right)=\left(M_{1} \cap L, M_{2} \cap L\right)^{1},
\end{gathered}
$$

утверждение справедливо для $i=1$. Предположим, что утверждение справедливо для $i=i_{0}$, где $i_{0}$ - некоторое целое положительное число, и покажем, что тогда утверждение справедливо и для $i=i_{0}+1$. Для этого замечаем, что

$$
\begin{gathered}
\left(M_{1}, M_{2}\right)^{i_{0}+1} \cap L=\left(\cap g \in M_{1}\left(\left(M_{1}, M_{2}\right)^{i_{0}} \cap\left(M_{2}, M_{1}\right)^{i_{0}}\right)^{g}\right) \cap L \\
\leq\left(M_{1}, M_{2}\right)^{i_{0}} \cap\left(\cap_{g \in M_{1} \cap L}\left(\left(\left(M_{2}, M_{1}\right)^{i_{0}}\right)^{g}\right)\right) \cap L \\
=\left(\left(M_{1}, M_{2}\right)^{i_{0}} \cap L\right) \cap\left(\cap_{g \in M_{1} \cap L}\left(\left(\left(M_{2}, M_{1}\right)^{i_{0}} \cap L\right)^{g}\right)\right) \\
\leq\left(M_{1} \cap L, M_{2} \cap L\right)^{i_{0}} \cap\left(\cap_{g \in M_{1} \cap L}\left(\left(\left(M_{2} \cap L, M_{1} \cap L\right)^{i_{0}}\right)^{g}\right)\right) \\
=\cap_{g \in M_{1} \cap L}\left(\left(\left(M_{1} \cap L, M_{2} \cap L\right)^{i_{0}} \cap\left(M_{2} \cap L, M_{1} \cap L\right)^{i_{0}}\right)^{g}\right)=\left(M_{1} \cap L, M_{2} \cap L\right)^{i_{0}+1} .
\end{gathered}
$$

В дальнейшем пусть $\left(G, M_{1}, M_{2}\right) \in \Pi$, $\operatorname{Soc}(G)$ - простая ортогональная группа $O_{n}(q)(n \geq 7$ и $n q$ нечетно) или $O_{n}^{\varepsilon}(q)(n \geq 8$ четно и $\varepsilon \in\{+,-\})$, где $q-$ степень простого числа $r$, $M_{1} \cap \operatorname{Soc}(G)$ - непараболическая подгруппа в $\operatorname{Soc}(G)$ и $M_{2}=M_{1}^{g}=g M_{1} g^{-1}$, где $g \in G$. Положим для краткости $L=\operatorname{Soc}(G)$ (мы считаем, что $L=\operatorname{Inn}(L) \unlhd G \leq A u t(L)), M=M_{1}$, $M_{0}=M \cap L, K_{1}=\left(M_{1}, M_{2}\right)^{2}, K_{2}=\left(M_{2}, M_{1}\right)^{2}, T_{1}=K_{1} \cap L, T_{2}=K_{2} \cap L, R_{1}=O_{p}\left(\left(M_{1}, M_{2}\right)^{1}\right)$ и $R_{2}=O_{p}\left(\left(M_{2}, M_{1}\right)^{1}\right)$. Ввиду [4, ч. I, предложение $1.1 ; 4$, ч. II, предложение 1.1] подгруппа $M$ 
есть $p$-локальная максимальная подгруппа в $G$ с $F^{*}(M)=O_{p}(M)$ для некоторого простого числа $p$ (отличного от $r$ ), $F^{*}\left(M_{0}\right)=O_{p}\left(M_{0}\right), G=M L, K_{1} K_{2} \leq R_{1} \cap R_{2} \leq O_{p}\left(M_{1}\right) \cap O_{p}\left(M_{2}\right)$, где $R_{1}=O_{p}\left(\left(M_{1}, M_{2}\right)^{1}\right), R_{2}=O_{p}\left(\left(M_{2}, M_{1}\right)^{1}\right)$ и $K_{1} \neq K_{2}$. Поскольку $G=M L$, будем считать, что $g \in L$. Пусть $V$ - векторное пространство размерности $n$ над полем $F=\mathbb{F}_{q}$ с невырожденной квадратичной формой, ассоциированное с ортогональной группой $L$. Естественным образом считаем, что $L=O(V) \leq P S O(V) \leq P G O(V) \leq P C G O(V) \leq P \Gamma O(V) \leq A u t(L)$, причем $G \leq P \Gamma O(V)$ при $L \nsucceq O_{8}^{+}(q)$ (мы используем обозначения из [8]).

Для произвольной подгруппы $H$ группы $P \Gamma O(V)$ через $\tilde{H}$ обозначается полный прообраз $H$ при естественном гомоморфизме $\Gamma O(V) \rightarrow P \Gamma O(V)$. Для произвольного элемента $h$ группы $P \Gamma O(V)$ через $\tilde{h}$ обозначается (некоторый) его прообраз при естественном гомоморфизме $\Gamma O(V) \rightarrow P \Gamma O(V)$. Для произвольного элемента $h$ группы $\Gamma O(V)$ через $\bar{h}$ обозначается его образ при естественном гомоморфизме $Г O(V) \rightarrow P \Gamma O(V)$.

Для векторного пространства мы используем как синонимы термины "одномерное подпространство" и "прямая".

Под графовым автоморфизмом группы $O_{8}^{+}(q)$ будем понимать ее автоморфизм, определенный в $\left[13\right.$, определение 2.5.13(b)], под стандартным графовым автоморфизмом группы $O_{8}^{+}(q)-$ ее автоморфизм, $A u t\left(O_{8}^{+}(q)\right)$-сопряженный с автоморфизмом из [13, теорема 2.5.1(d)].

Лемма 1.2. Выполняется одно из следующих утверждений:

(i) подгруппы $K_{1}$ и $K_{2}$ имеют нетривиальные пересечения с $L$;

(ii) $L \cong O_{8}^{+}(q)$, q нечетно, $p=2, L E=\operatorname{Inndiag}(L)<G$ для некоторой четверной подгруппы $E, M=N_{L}(E)$ и $G$ содержит графовый автоморфизм порядка 3 группы $L$.

Д о к а з а т е л ь с т в о. Предположим, что п. (i) не верен, и пусть $K_{1} \cap L=1$ (случай $K_{2} \cap L=1$ рассматривается аналогично). Поскольку $K_{1}-$ неединичная нормальная $p$-подгруппа в $M$, существует минимальная неединичная нормальная в $M$ подгруппа $E$, содержащаяся в $K_{1}$. Ясно, что $M_{0}=C_{L}(E)$.

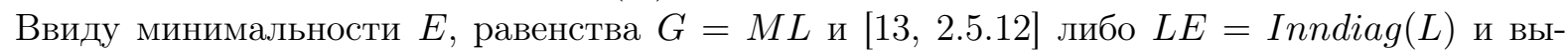
полняется п. (ii), либо подгруппа $E$ порождается некоторым элементом $x$ порядка $p$. Таким образом, можно считать, что выполняется второй случай и, следовательно, $M_{0}=C_{L}(x)$ и $M=N_{G}(\langle x\rangle)$. Элемент $x$ индуцирует на $L$ внешний автоморфизм порядка $p$. Согласно [13, 2.5.12] этот автоморфизм внутренне-диагональный, графовый, полевой или графово-полевой. Ввиду $[13,4.5 .1,4.7 .3,4.8 .2,4.9 .1]$ и максимальности в $G$ подгруппы $M$ это противоречит равенству $F^{*}\left(M_{0}\right)=O_{p}\left(M_{0}\right)$.

\section{2. Доказательство теоремы в случае, когда группа $L$ не изоморфна $O_{8}^{+}(q)$}

В данном разделе предполагается, что группа $L$ не изоморфна $O_{8}^{+}(q)$.

Согласно теореме 2.2.19 из [8], являющейся уточненной версией теоремы Ашбахера [5], группа $M$ принадлежит одному из классов Ашбахера $C_{i}(G)$ геометрических подгрупп группы $G$ для $1 \leq i \leq 8$, которые мы определяем как факторгруппы по $Z(\tilde{G})$ геометрических подгрупп группы $\tilde{G}$ из соответствующих ее классов $\mathcal{C}_{i}$, определенных в [8, разд. 2.2]. Возможное строение $\widetilde{M_{1}} \cap \Omega(V)$ приведено в [8, табл. 2.3, 2.5-2.11].

Далее мы рассмотрим возможность включения подгруппы $M$ в каждый класс $C_{i}(G)$, $1 \leq i \leq 8$, в отдельности.

Лемма 2.1. $M \notin C_{1}(G)$.

Д ок а з а т е ль с т в о. Предположим, что лемма не верна. Тогда ввиду равенства $F^{*}\left(M_{0}\right)=O_{p}\left(M_{0}\right)$ и [8, табл. $2.3 ; 15$, разд. $2.9,4.1 ; 13$, теорема 4.5.1] имеем $L \cong O_{7}(3)$, $M_{0} \cong S O_{3}(3) \times S O_{4}^{+}(3) \cong S_{4} \times\left(\left(S L_{2}(3) \circ S L_{2}(3)\right) .2\right)$. 
Ввиду леммы 1.2 подгруппы $T_{1}$ и $T_{2}$ нетривиальны. Положим $Z_{i}=Z\left(O_{2}\left(M_{i} \cap L\right)\right)$ и $\left\langle z_{i}\right\rangle=$ $\left[O_{2}\left(M_{i} \cap L\right), O_{2}\left(M_{i} \cap L\right)\right]$ для $i \in\{1,2\}$. Можно считать, что $z_{1}^{g}=z_{2}$. Поскольку $T_{1} T_{2} \leq R_{1} \cap R_{2}$, имеем $\left[T_{i}, T_{i}\right] \leq\left\langle z_{1}\right\rangle \cap\left\langle z_{2}\right\rangle=1$ для $i \in\{1,2\}$. Поэтому подгруппы $T_{1}$ и $T_{2}$ абелевы, а значит, подгруппы $Z_{1} T_{1}$ и $Z_{2} T_{2}$ абелевы.

Покажем, что $T_{i} \leq Z_{i}$ для $i \in\{1,2\}$. Предположим противное, т. е. что $T_{i} \not \leq Z_{i}$ для некоторого $i \in\{1,2\}$. Подгруппа $M_{i} \cap L$ действует неприводимо на $O_{2}\left(M_{i} \cap L\right) / Z_{i}$, следовательно, $Z_{i} T_{i}=O_{2}\left(M_{i} \cap L\right)$, что противоречит некоммутативности подгруппы $O_{2}\left(M_{i} \cap L\right)$. Поэтому наше утверждение доказано.

Ввиду [6] подгруппа $M_{0}$ содержит некоторую силовскую 2-подгруппу $S$ группы $L$ и $N_{L}(S)<M_{0}$. Теорема Бернсайда [12, теорема 7.1.1] влечет, что подгруппа $\left\langle z_{1}\right\rangle$ сильно замкнута в $Z(S)$ относительно $L$. Поэтому подгруппа $\left\langle z_{1}\right\rangle$ сильно замкнута в $Z\left(O_{2}(M) \cap L\right)$ относительно $L$, а следовательно, подгруппа $\left\langle z_{2}\right\rangle$ сильно замкнута в $Z\left(O_{2}\left(M_{2}\right) \cap L\right)$ относительно $L$.

Предположим, что $T_{2}$ не лежит в $Z_{1}$. Тогда $C_{O_{2}(M) \cap L}\left(T_{2}\right)=Z_{1} T_{2} \leq M_{1} \cap M_{2}$ и, следовательно, $Z_{1}<Z_{1} T_{2} \leq R_{1}$. Подгруппа $M_{1} \cap L$ действует неприводимо на $O_{2}\left(M_{1} \cap L\right) / Z_{1}$, поэтому $R_{1} \cap L=O_{2}\left(M_{1} \cap L\right)$ и, следовательно, коммутант и центр подгруппы $R_{1} \cap L$ равны $\left\langle z_{1}\right\rangle$ и $Z_{1}$ соответственно. Подгруппа $T_{2}$ нормальна в $R_{1}$ и не содержится в $Z_{1}$, следовательно, $\left\langle z_{1}\right\rangle \leq\left[T_{2}, R_{1} \cap L\right] \leq T_{2}$. Но тогда по предыдущему абзацу имеем $\left\langle z_{1}\right\rangle=\left\langle z_{2}\right\rangle$, что невозможно.

Итак, $T_{2}$ лежит в $Z_{1}$. Ясно, что $T_{2}<Z_{1}$. Аналогично получаем, что $T_{1}<Z_{2}$. Тогда $\left|T_{1}\right|,\left|T_{2}\right| \leq 4$. Если $\left|T_{1}\right|=2$, то $T_{1}=\left\langle z_{1}\right\rangle<Z_{2}$ и, следовательно, $\left\langle z_{1}\right\rangle=\left\langle z_{2}\right\rangle$, что невозможно. Аналогично получаем, что $\left|T_{2}\right| \neq 2$. Поэтому $T_{i}$ - единственная четверная минимальная нормальная подгруппа в $M_{i} \cap L$ для $i \in\{1,2\}$. Но тогда $Z_{1} \cap Z_{2}=T_{1}=T_{2}$, что невозможно.

Лемма 2.2. $M \notin C_{6}(G)$.

Д о к а з а т е л ь с т в о. Предположим, что лемма не верна. Тогда согласно [8, табл. 2.9] имеем, что $O_{p}\left(M_{0}\right)$ - (нетривиальная) элементарная абелева группа, на которой группа $M_{0} / O_{p}\left(M_{0}\right)$ действует неприводимо. Так как согласно лемме 1.2 для каждого $i \in\{1,2\}$ нормальная $p$-подгруппа $T_{i}$ группы $M_{i}$ неединична, то отсюда следует, что $T_{i}=O_{p}\left(M_{i} \cap L\right)$ для каждого $i \in\{1,2\}$ и, в частности, $\left|T_{1}\right|=\left|T_{2}\right|$. Но $T_{2} \leq O_{p}\left(M_{1}\right) \cap L=O_{p}\left(M_{1} \cap L\right)$. Следовательно, $T_{1}=T_{2}$, что невозможно.

Лемма 2.3. $M \notin C_{i}(G)$ для $i \in\{3,4,5,7,8\}$.

Д о к а з а т е л ь с т в о. Лемма следует из равенства $F^{*}\left(M_{0}\right)=O_{p}\left(M_{0}\right)$ и $[8$, табл. 2.6-2.8, $2.10,2.11 ; 15$, разд. $2.9,4.3,4.4,4.5,4.7,4.8]$.

Лемма 2.4. $M \notin C_{2}(G)$.

Д о к а з а т е ль с т в о. Предположим, что лемма не верна. Согласно [8, теорема 2.2.19.(i).(b)] подгруппа $\widetilde{M}_{1}$ есть стабилизатор в $G$ ортогонального разложения $V=$ $V_{1}+\ldots+V_{t}$ пространства $V$ на изометричные невырожденные подпространства $V_{1}, \ldots, V_{t}$, где $t \geq 2$ и $\operatorname{dim} V_{i}=m=n / t$ для всех $1 \leq i \leq t$, одного из перечисленных в [8, табл. 2.4] типов. В [8, табл. 2.5] для каждого из этих типов указано строение группы $M_{0}=M_{1} \cap L$ (точнее, группы $\widetilde{M}_{0} \cap \Omega(V)$ - стабилизатора разложения в группе $\Omega(V)$ ), см. [8, теорема 2.2.19.(i).(b)]. Из этого строения и равенства $F^{*}\left(M_{0}\right)=O_{p}\left(M_{0}\right)$ следует, что $m \leq 4$ и $t \geq 3$. Отметим также, что согласно [8, табл. 2.5] (см. также [8, лемма 2.2.4]) группа $M_{0}$ индуцирует на множестве $\left\{V_{1}, \ldots, V_{t}\right\}$ симметрическую или знакопеременную группу подстановок. Кроме того, в силу леммы 1.2 подгруппы $K_{1} \cap L$ и $K_{2} \cap L$ нетривиальны.

Положим $C_{1}=\left\{h \in G: h\left(V_{i}\right)=V_{i}\right.$ для всех $\left.1 \leq i \leq t\right\}$ - ядро действия $M_{1}$ на множестве $\left\{V_{1}, \ldots, V_{t}\right\}, C_{2}=g C_{1} g^{-1}-$ ядро действия $M_{2}$ на множестве $\left\{g\left(V_{1}\right), \ldots, g\left(V_{t}\right)\right\}$. Имеет место включение

$$
O_{p}\left(M_{1}\right) \leq C_{1}
$$


Действительно, из строения группы $M_{0}$ (см. [8, табл. 2.5]) легко следует справедливость (2.1) в случае $m \geq 3$, а также в случае $t \geq 5$ (в последнем случае достаточно воспользоваться тем, что $M_{0}$ индуцирует на $\left\{V_{1}, \ldots, V_{t}\right\}$ либо симметрическую группу, либо знакопеременную группу). Что касается случая, когда $m \leq 2$ и $t \leq 4$, то он невозможен в силу $n \geq 7$ и $L \neq O_{8}^{+}(q)$.

Поскольку $K_{1}$ и $K_{2}$ являются подгруппами группы $O_{p}\left(M_{1}\right) \cap O_{p}\left(M_{2}\right)$, то из $(2.1)$ следует

$$
\left\langle K_{1}, K_{2}\right\rangle \leq C_{1} \cap C_{2} .
$$

Так как $\left\{V_{1}, \ldots, V_{t}\right\} \neq\left\{g\left(V_{1}\right), \ldots, g\left(V_{t}\right)\right\}$ (поскольку $M_{1} \neq M_{2}$ ), будем, не теряя общности, предполагать, что $g\left(V_{1}\right) \notin\left\{V_{1}, \ldots, V_{t}\right\}$. Тогда найдется $w \in \tilde{g}\left(V_{1}\right)$ такой, что $w=w_{1}+\ldots+w_{t}$, где $w_{i} \in V_{i}$ для каждого $1 \leq i \leq t$, причем $w_{k} \neq 0 \neq w_{l}$ для некоторых $1 \leq k<l \leq t$.

С л у ч а й $m=1$. Поскольку $1 \neq K_{1} \cap L \unlhd M_{1}$ (см. лемму 1.2), причем $K_{1} \leq C_{1}$ (см. (2.2)), а $M_{1}$ индуцирует на множестве $\left\{V_{1}, \ldots, V_{t}\right\}$ группу подстановок, содержащую знакопеременную группу, то согласно [8, табл. 2.5] некоторый элемент $h \in K_{1} \cap L$ не стабилизирует подпространство $\left\langle w_{k}+w_{l}\right\rangle$ векторного пространства $V$. Но тогда (учитывая, что $h \in C_{1}$ ) элемент $h$ не стабилизирует и подпространство $\left\langle w_{1}+\ldots+w_{t}\right\rangle=\langle w\rangle=g\left(V_{1}\right)$ векторного пространства $V$. Полученное противоречие с $K_{1} \leq C_{2}$ (см. (2.2)) завершает рассмотрение случая $m=1$.

С л у ч а й $m=2$. Согласно [8, табл. 2.5$]$ для $\tilde{G}$ и $\widetilde{M}_{1}$ имеет место случай $\mathbf{O}^{\varepsilon}$, тип $G O_{2}^{\varepsilon_{1}}(q) 2 S_{t}$. Далее, согласно [8, табл. 2.4] $V_{i}$ невырождено и $\varepsilon_{1}=\operatorname{sgn}\left(V_{i}\right)$ для каждого $1 \leq i \leq t$, a $\varepsilon=-$ лишь при $\varepsilon_{1}=-$ и нечетном $t$.

Если $\varepsilon_{1}=+$, то, с учетом [8, табл. 2.5] и $O(V)=[P G O(V), P G O(V)]$, для каждого $1 \leq i \leq t$ стабилизатор $V_{i}$ в группе $M_{1} \cap O(V)=M_{0}$, индуцирует на множестве одномерных подпространств пространства $V_{i}$ группу $P G O\left(V_{i}\right) \cong P G O_{2}^{+}(q) \cong D_{(2, q)(q-1)}$. Более того, для произвольных различных $1 \leq i_{1}, i_{2} \leq t$ в $M_{0}$ имеется подгруппа $R_{i_{1}, i_{2}}$, стабилизирующая все одномерные подпространства пространств $V_{j}, 1 \leq j \leq t, i_{1} \neq j \neq i_{2}$, и индуцирующая на множестве одномерных подпространств пространства $V_{i_{1}}$ группу $P G O\left(V_{i_{1}}\right)$, а на множестве одномерных подпространств пространства $V_{i_{2}}$ группу $P G O\left(V_{i_{2}}\right)$. Отметим, что группа $P G O\left(V_{i}\right), 1 \leq i \leq t$, действует транзитивно на множестве (из $q-1$ ) несингулярных прямых $V_{i}$ в случае четного $q$, а в случае нечетного $q$ имеет на этом множестве в точности две орбиты, длиной $(q-1) / 2$ каждая, являющиеся множеством несингулярных прямых $V_{i}$, значения квадратичной формы на векторах которых являются квадратами в поле $F$, и множеством прямых $V_{i}$, значения квадратичной формы на ненулевых векторах которых не являются квадратами в поле $F$.

Если $\varepsilon_{1}=-$, то для каждого $1 \leq i \leq t$ подпространство $V_{i}$ пространства $V$ не содержит ненулевых сингулярных векторов и, с учетом [8, табл. 2.5] и $O(V)=[P G O(V), P G O(V)]$, ограничение на множество прямых $V_{i}$ стабилизатора $V_{i}$ в группе $M_{1} \cap O(V)=M_{0}$, есть группа $P G O\left(V_{i}\right) \cong P G O_{2}^{-}(q) \cong D_{(2, q)(q+1)}$, индуцированная группой $G O\left(V_{i}\right) \cong G O_{2}^{-}(q) \cong D_{2(q+1)}$ и действующая транзитивно как на множестве прямых $V_{i}$, значения квадратичной формы на векторах которых являются квадратами в поле $F$, так и, в случае нечетного $q$, на множестве прямых $V_{i}$, значения квадратичной формы на ненулевых векторах которых не являются квадратами в поле $F$.

Из равенства $F^{*}\left(M_{1} \cap L\right)=O_{p}\left(M_{1} \cap L\right)$ с учетом [8, предложение 2.3.6] и [11] следует, что при $\varepsilon_{1}=+$ либо $q=9$ (при этом $p=2$ ), либо $q-$ простое нечетное число $>3$ и $q-1-$ степень 2 (при этом $p=2$ ), либо $q$ - степень 2 и $q-1-$ простое нечетное число (при этом $p=q-1$ ), а при $\varepsilon_{1}=-$ либо $q=8$ (при этом $p=3$ ), либо $q-$ простое нечетное число и $q+1-$ степень 2 (при этом $p=2$ ), либо $q$ - степень 2 и $q+1$ - простое нечетное число (при этом $p=q+1)$.

Рассмотрим отдельно случаи нечетного и четного $q$.

Предположим, что q нечетно ( вида $M_{0}$ (см. [8, табл. 2.5]) и $1 \neq K_{1} \cap L \unlhd M_{1}$ (см. лемму 1.2) следует существование для произвольных различных $1 \leq i_{1}, i_{2} \leq t$ элемента $h_{i_{1}, i_{2}}$ группы $\widetilde{K_{1}} \cap \tilde{L}$, который действует тождественно на каждом из подпространств $V_{i}, 1 \leq i \leq t, i_{1} \neq i \neq i_{2}$, векторного пространства $V$ и 
инвертирует подпространства $V_{i_{1}}$ и $V_{i_{2}}$. Кроме того, из вида $M_{0}$ и $1 \neq g^{-1}\left(K_{2} \cap L\right) g \unlhd M_{1}$ (см. лемму 2.2) следует, что $\tilde{g} h_{i_{1}, i_{2}} \tilde{g}^{-1} \in \widetilde{K_{2}} \cap \tilde{L} \leq O_{2}\left(\left(M_{1}, M_{2}\right)^{1}\right)$ для всех $1 \leq i_{1}, i_{2} \leq t, i_{1} \neq i_{2}$. В частности, учитывая (2.2), для произвольных различных $1 \leq i_{1}, i_{2} \leq t$ получаем, что элементы $h_{i_{1}, i_{2}}$ и $\tilde{g} h_{i_{1}, i_{2}} \tilde{g}^{-1}$ содержатся в $\widetilde{C_{1}} \cap \widetilde{C_{2}} \cap \tilde{L}$.

Покажем, что если $\tilde{g}\left(V_{i}\right) \notin\left\{V_{1}, \ldots, V_{t}\right\}$, где $1 \leq i \leq t$, то для некоторых $1 \leq i^{\prime}<i^{\prime \prime} \leq t$ имеем $\operatorname{dim}\left(\tilde{g}\left(V_{i}\right) \cap V_{i^{\prime}}\right)=1=\operatorname{dim}\left(\tilde{g}\left(V_{i}\right) \cap V_{i^{\prime \prime}}\right)$, и что, аналогично, если $V_{i} \notin\left\{\tilde{g}\left(V_{1}\right), \ldots, \tilde{g}\left(V_{t}\right)\right\}$, где $1 \leq i \leq t$, то для некоторых $1 \leq i^{\prime}<i^{\prime \prime} \leq t$ имеем $\operatorname{dim}\left(V_{i} \cap \tilde{g}\left(V_{i^{\prime}}\right)\right)=1=\operatorname{dim}\left(V_{i} \cap \tilde{g}\left(V_{i^{\prime \prime}}\right)\right)$.

Следующие рассуждения аналогичны рассуждениям из доказательства леммы 2.8 (в случае $m=2)$ в [4, ч. III]. Пусть $\tilde{g}\left(V_{i}\right) \notin\left\{V_{1}, \ldots, V_{t}\right\}$, где $1 \leq i \leq t$. Тогда найдется $v \in \tilde{g}\left(V_{i}\right)$ такой, что $v=v_{1}+\ldots+v_{t}$, где $v_{j} \in V_{j}$ для всех $1 \leq j \leq t$, причем $v_{i^{\prime}} \neq 0 \neq v_{i^{\prime \prime}}$ для некоторых $1 \leq i^{\prime}<i^{\prime \prime} \leq t$. Докажем, что $\tilde{g}\left(V_{i}\right) \cap V_{i^{\prime}}=\left\langle v_{i^{\prime}}\right\rangle$ и $\tilde{g}\left(V_{i}\right) \cap V_{i^{\prime \prime}}=\left\langle v_{i^{\prime \prime}}\right\rangle$, для чего, очевидно, достаточно доказать, что $\tilde{g}\left(V_{i}\right)$ содержит $v_{i^{\prime}}$ и $v_{i^{\prime \prime}}$. Но с учетом $h_{i^{\prime}, i^{\prime \prime}} \in \widetilde{C_{2}}$ имеем $v_{i^{\prime}}+v_{i^{\prime \prime}}=\frac{1}{2}\left(v-h_{i^{\prime}, i^{\prime \prime}}(v)\right) \in \tilde{g}\left(V_{i}\right)$, что с учетом $h_{i^{\prime}, j^{\prime}} \in \widetilde{C_{2}}$, где $1 \leq j^{\prime} \leq t, i_{1}^{\prime} \neq j^{\prime} \neq i^{\prime \prime}$, влечет $v_{i^{\prime}}=\frac{1}{2}\left(v_{i^{\prime}}+v_{i^{\prime \prime}}-h_{i^{\prime}, j^{\prime}}\left(v_{i^{\prime}}+v_{i^{\prime \prime}}\right)\right) \in \tilde{g}\left(V_{i}\right)$ и $v_{i^{\prime \prime}}=\frac{1}{2}\left(v_{i^{\prime}}+v_{i^{\prime \prime}}+h_{i^{\prime}, j^{\prime}}\left(v_{i^{\prime}}+v_{i^{\prime \prime}}\right)\right) \in \tilde{g}\left(V_{i}\right)$, завершая доказательство первой части утверждения. Вторая часть утверждения доказывается сходным образом. Пусть $V_{i} \notin\left\{\tilde{g}\left(V_{1}\right), \ldots, \tilde{g}\left(V_{t}\right)\right\}$, где $1 \leq i \leq t$. Тогда найдется $v \in V_{i}$ такой, что $v=v_{1}+\ldots+v_{t}$, где $v_{j} \in \tilde{g}\left(V_{j}\right)$ для всех $1 \leq j \leq t$, причем $v_{i^{\prime}} \neq 0 \neq v_{i^{\prime \prime}}$ для некоторых $1 \leq i^{\prime}<i^{\prime \prime} \leq t$. Докажем, что $V_{i} \cap \tilde{g}\left(V_{i^{\prime}}\right)=\left\langle v_{i^{\prime}}\right\rangle$ и $V_{i} \cap \tilde{g}\left(V_{i^{\prime \prime}}\right)=\left\langle v_{i^{\prime \prime}}\right\rangle$, для чего, очевидно, достаточно доказать, что $V_{i}$ содержит $v_{i^{\prime}}$ и $v_{i^{\prime \prime}}$. Но с учетом $\tilde{g} h_{i^{\prime}, i^{\prime \prime}} \tilde{g}^{-1} \in \widetilde{C}_{1}$ (выше было замечено, что $\tilde{g} h_{i_{1}, i_{2}} \tilde{g}^{-1} \in \widetilde{C_{1}}$ для произвольных $1 \leq i_{1}, i_{2} \leq t, i_{1} \neq i_{2}$ ) имеем $v_{i^{\prime}}+v_{i^{\prime \prime}}=\frac{1}{2}\left(v-\tilde{g} h_{i^{\prime}, i^{\prime \prime}} \tilde{g}^{-1}(v)\right) \in V_{i}$, что с учетом $\tilde{g} h_{i^{\prime}, j^{\prime}} \tilde{g}^{-1} \in \widetilde{C_{1}}$, где $1 \leq j^{\prime} \leq t, i_{1}^{\prime} \neq j^{\prime} \neq i^{\prime \prime}$, влечет $v_{i^{\prime}}=\frac{1}{2}\left(v_{i^{\prime}}+v_{i^{\prime \prime}}-\tilde{g} h_{i^{\prime}, j^{\prime}} \tilde{g}^{-1}\left(v_{i^{\prime}}+v_{i^{\prime \prime}}\right)\right) \in V_{i}$ и $v_{i^{\prime \prime}}=\frac{1}{2}\left(v_{i^{\prime}}+v_{i^{\prime \prime}}+\tilde{g} h_{i^{\prime}, j^{\prime}} \tilde{g}^{-1}\left(v_{i^{\prime}}+v_{i^{\prime \prime}}\right)\right) \in V_{i}$, завершая доказательство утверждения.

Из доказанного утверждения вытекает справедливость следующих утверждений: $w=$ $w_{k}+w_{l}, \tilde{g}\left(V_{1}\right)=\left\langle w_{k}, w_{l}\right\rangle,\left\langle w_{k}\right\rangle=V_{k} \cap \tilde{g}\left(V_{1}\right)$ и $\left\langle w_{l}\right\rangle=V_{l} \cap \tilde{g}\left(V_{1}\right)$, причем $w_{k}$ и $w_{l}$ не являются сингулярными (поскольку $\left(w_{k}, w_{l}\right)=0$, a $\tilde{g}\left(V_{1}\right)$ невырождено) и лишь $\left\langle w_{k}\right\rangle$ и $\left\langle w_{l}\right\rangle$ являются одномерными подпространствами как некоторых $V_{i}, 1 \leq i \leq t$, так и $\tilde{g}\left(V_{1}\right)$. Кроме того, поскольку $\operatorname{dim}\left(V_{k} \cap g\left(V_{1}\right)\right)=1$, из доказанного утверждения вытекает существование такого $2 \leq i_{0} \leq t$, что $\operatorname{dim}\left(V_{k} \cap g\left(V_{i_{0}}\right)\right)=1$.

Пусть $1 \leq j \leq t, k \neq j \neq l$. Тогда элемент $h_{k, j}$ инвертирует вектор $w_{k}$ и стабилизирует вектор $w_{l}$. Ясно, что $h_{k, j}$ нетривиально действует на множестве одномерных подпространств из $\tilde{g}\left(V_{1}\right)$, и, следовательно, не стабилизирует отличных от $\left\langle w_{k}\right\rangle,\left\langle w_{l}\right\rangle$ одномерных подпространств из $\tilde{g}\left(V_{1}\right)$.

Если $\varepsilon_{1}=+$ и $q>5$ (и тогда, см. выше, $q \geq 9$ ) или $\varepsilon_{1}=-$ и $q>3$ (и тогда, см. выше, $q \geq 7)$, то для группы $R_{1, j^{\prime}}^{g}$, где $2 \leq j^{\prime} \leq t$ и $j^{\prime} \neq i_{0}$, стабилизирующей все одномерные подпространства пространств $\tilde{g}\left(V_{i}\right)$, где $1 \leq i \leq t$ и $1 \neq i \neq j^{\prime}$, содержащая $\left\langle w_{k}\right\rangle$ орбита на множестве одномерных подпространств пространства $\tilde{g}\left(V_{1}\right)$ имеет длину $>2$, и потому для некоторого $h \in R_{1, j^{\prime}}^{g}$ элемент $\bar{h}_{k, j}$ не стабилизирует $h\left(\left\langle w_{k}\right\rangle\right)$, т. е.

$$
h^{-1} \bar{h}_{k, j} h\left(\left\langle w_{k}\right\rangle\right) \neq\left\langle w_{k}\right\rangle \text {. }
$$

В силу $h \in R_{1, j^{\prime}}^{g} \leq M_{1}^{g}=M_{2}$ и $\bar{h}_{k, j} \in K_{1} \leq\left(M_{2}, M_{1}\right)^{1}$ имеем $h^{-1} \bar{h}_{k, j} h \leq\left(M_{2}, M_{1}\right)^{1} \leq M_{1}$. Таким образом, элемент $\tilde{h}^{-1} h_{k, j} \tilde{h}$ стабилизирует разложение $V=V_{1}+\ldots+V_{t}$. Кроме того, элемент $\tilde{h}^{-1} h_{k, j} \tilde{h}$ стабилизирует подпространство $\tilde{g}\left(V_{1}\right)$ пространства $V$, поскольку элементы $\tilde{h}$ и $h_{k, j}$ стабилизируют $\tilde{g}\left(V_{1}\right)$. Так как лишь $\left\langle w_{k}\right\rangle$ и $\left\langle w_{l}\right\rangle$ являются одномерными подпространствами как некоторых $V_{i}, 1 \leq i \leq t$, так и $\tilde{g}\left(V_{1}\right)$, из $(2.3)$ следует, что $\tilde{h}^{-1} h_{k, j} \tilde{h}\left(\left\langle w_{k}\right\rangle\right)=\left\langle w_{l}\right\rangle$, а значит, $\tilde{h}^{-1} h_{k, j} \tilde{h}\left(V_{k}\right)=V_{l}$. Однако последнее равенство противоречит тому, что, поскольку элемент $\tilde{h}$ стабилизирует каждое одномерное подпространство пространства $\tilde{g}\left(V_{i_{0}}\right)$ и элемент $h_{k, j}$ стабилизирует каждое одномерное подпространство пространства $V_{k}$, имеет место равенство $\tilde{h}^{-1} h_{k, j} \tilde{h}\left(V_{k} \cap \tilde{g}\left(V_{i_{0}}\right)\right)=V_{k} \cap \tilde{g}\left(V_{i_{0}}\right)$. 
Пусть $\varepsilon_{1}=+$ и $q=5$. Тогда для $1 \leq i \leq t$ группа $P G O\left(V_{i}\right)$ имеет на множестве несингулярных прямых пространства $V_{i}$ две орбиты длины 2. А именно, если $\{e, f\}-$ гиперболическая пара гиперболической плоскости $V_{i}$, то $P G O\left(V_{i}\right)$-орбитами на множестве несингулярных прямых $V_{i}$ являются $X_{i}^{-}=\{\langle e+f\rangle,\langle e-f\rangle\}$ и $X_{i}^{+}=\{\langle e+2 f\rangle,\langle e-2 f\rangle\}$, т. е. в точности все пары ортогональных несингулярных прямых $V_{i}$. Если $v-$ ненулевой вектор прямой из $X_{i}^{-}$, то $(v, v)$ не есть квадрат в поле $F$, а если $v$ - вектор прямой из $X_{i}^{+}$, то $(v, v)$ есть квадрат в поле $F$.

Сходная ситуация имеет место при $\varepsilon_{1}=-$ и $q=3$. В этом случае для $1 \leq i \leq t$ группа $P G O\left(V_{i}\right)$ имеет на множестве прямых пространства $V_{i}$ две орбиты $X_{i}^{-}$и $X_{i}^{+}$длины 2 со следующими свойствами: если $v$ - ненулевой вектор прямой из $X_{i}^{-}$, то $(v, v)$ не есть квадрат в поле $F$, а если $v$ - вектор прямой из $X_{i}^{+}$, то $(v, v)$ есть квадрат в поле $F$. При этом множества $X_{i}^{-}, X_{i}^{+}$и только они являются парами ортогональных прямых $V_{i}$.

Дальнейшие рассуждения применимы как в случае $\varepsilon_{1}=+$ и $q=5$, так и в случае $\varepsilon_{1}=-$ и $q=3$. Для $1 \leq i \leq t$ ограничение на множество прямых $V_{i}$ стабилизатора этого множества в группе $M_{1}$ содержит группу $P G O\left(V_{i}\right)$ (см. выше), но отлично от $P G O\left(V_{i}\right)$, что можно усмотреть из $\left[15\right.$, предложение 6.2.11] и [8]. (Если ограничение на множество прямых $V_{i}$ стабилизатора этого множества в группе $M_{1}$ есть группа $P G O\left(V_{i}\right)$, то группа $\widetilde{M}_{1}$ содержится в стабилизаторе ортогонального разложения $V$ в сумму образов $\left\langle w_{k}\right\rangle$ под действием элементов из $\tilde{G}$, причем содержится собственным образом, поскольку этот стабилизатор индуцирует на множестве слагаемых разложения группу, содержащую знакопеременную группу $A_{n}$, а группа $M_{1}$ не имеет секций, изоморфных $A_{n}$.) Следовательно, для $1 \leq i \leq t$ ограничение на множество прямых $V_{i}$ стабилизатора этого множества в группе $M_{1}$ есть группа $P C G O\left(V_{i}\right)$. Но тогда, учитывая, что $\widetilde{M}_{0}$ индуцирует на множестве слагаемых разложения $V=V_{1}+\ldots+V_{t}$ симметрическую группу (см. [8, табл. 2.5]), заключаем, что в $M_{1}$ имеется элемент $h \in P C G O(V) \backslash P G O(V)$, который для каждого $1 \leq i \leq t$ оставляет на месте множество прямых подпространства $V_{i}$. Элемент $h$ меняет при этом местами множества $X_{i}^{-}$и $X_{i}^{+}$для каждого $1 \leq i \leq t$. Действительно, в противном случае имеются такие $1 \leq i_{1}, i_{2} \leq t, i_{1} \neq i_{2}$, что $h$ меняет местами множества $X_{i_{1}}^{-}$и $X_{i_{1}}^{+}$, а множества $X_{i_{2}}^{-}$и $X_{i_{2}}^{+}$стабилизирует. Но в группе $\widetilde{M}_{0}$ найдется элемент, меняющий местами $V_{i_{1}}$ и $V_{i_{2}}$, и коммутатор этого элемента и элемента $\tilde{h}$ меняет местами множества $X_{i_{2}}^{-}$и $X_{i_{2}}^{+}$, что противоречит $\left[\widetilde{M}_{0}, \tilde{h}\right] \leq \tilde{L}$.

Пусть $\tilde{g}\left(V_{i}\right) \notin\left\{V_{1}, \ldots, V_{t}\right\}$, где $1 \leq i \leq t$. Тогда, как было доказано выше, найдутся $1 \leq$ $i^{\prime}, i^{\prime \prime} \leq t, i^{\prime} \neq i^{\prime \prime}$, такие, что $\operatorname{dim}\left(\tilde{g}\left(V_{i}\right) \cap V_{i^{\prime}}\right)=1=\operatorname{dim}\left(\tilde{g}\left(V_{i}\right) \cap V_{i^{\prime \prime}}\right)$. Покажем, что для некоторого $1 \leq i^{*} \leq t, i^{*} \neq i$, также имеем $\operatorname{dim}\left(\tilde{g}\left(V_{i^{*}}\right) \cap V_{i^{\prime}}\right)=1=\operatorname{dim}\left(\tilde{g}\left(V_{i^{*}}\right) \cap V_{i^{\prime \prime}}\right)$, причем $\left\{\tilde{g}\left(V_{i}\right) \cap\right.$ $\left.V_{i^{\prime}}, \tilde{g}\left(V_{i^{*}}\right) \cap V_{i^{\prime}}\right\}=X_{i^{\prime}}^{\xi}$ и $\left\{\tilde{g}\left(V_{i}\right) \cap V_{i^{\prime \prime}}, \tilde{g}\left(V_{i^{*}}\right) \cap V_{i^{\prime \prime}}\right\}=X_{i^{\prime \prime}}^{\xi}$ для некоторого $\xi \in\{-,+\}$.

Так как $V_{i^{\prime}} \notin\left\{\tilde{g}\left(V_{1}\right), \ldots, \tilde{g}\left(V_{t}\right)\right\}$ (поскольку $\operatorname{dim}\left(\tilde{g}\left(V_{i}\right) \cap V_{i^{\prime}}\right)=1$ ), то из доказанного выше следует существование такого $1 \leq i^{*} \leq t, i^{*} \neq i$, что $\operatorname{dim}\left(\tilde{g}\left(V_{i^{*}}\right) \cap V_{i^{\prime}}\right)=1$. Ясно, что $\tilde{g}\left(V_{i}\right) \cap V_{i^{\prime}}$ и $\tilde{g}\left(V_{i^{*}}\right) \cap V_{i^{\prime}}$ несингулярны (поскольку $V_{i^{\prime}}=\left\langle\tilde{g}\left(V_{i}\right) \cap V_{i^{\prime}}, \tilde{g}\left(V_{i^{*}}\right) \cap V_{i^{\prime}}\right\rangle$ невырождено). Пусть $j^{\prime} \in\{1, \ldots, t\} \backslash\left\{i, i^{*}\right\}$. Тогда элемент $\tilde{g} h_{i, j^{\prime}} \tilde{g}^{-1}$ из $\widetilde{K_{2}} \cap \tilde{L} \leq \widetilde{O_{2}\left(\left(M_{1}, M_{2}\right)^{1}\right)} \cap \tilde{L}$ (см. выше) инвертирует $\tilde{g}\left(V_{i}\right)$ и централизует $\tilde{g}\left(V_{i^{*}}\right)$, а следовательно, меняет местами несингулярные прямые подпространства $V_{i^{\prime}}$ пространства $V$, отличные от $\tilde{g}\left(V_{i}\right) \cap V_{i^{\prime}}$ и $\tilde{g}\left(V_{i^{*}}\right) \cap V_{i^{\prime}}$. Поскольку определенный выше элемент $h$ группы $M_{1}$ меняет местами множества $X_{i}^{-}$и $X_{i}^{+}$, отсюда следует, что содержащийся в $\widehat{O_{2}\left(\left(M_{1}, M_{2}\right)^{1}\right)} \cap \tilde{L} \leq \widetilde{C_{1}} \cap \widetilde{M_{2}} \cap \tilde{L}$ элемент $\tilde{h} \tilde{g} h_{i, j^{\prime}} \tilde{g}^{-1} \tilde{h}^{-1}$ меняет местами прямые $\tilde{g}\left(V_{i}\right) \cap V_{i^{\prime}}$ и $\tilde{g}\left(V_{i^{*}}\right) \cap V_{i^{\prime}}$. Но тогда элемент $\tilde{h} \tilde{g} h_{i, j^{\prime}} \tilde{g}^{-1} \tilde{h}^{-1}$ меняет местами $\tilde{g}\left(V_{i}\right)$ и $\tilde{g}\left(V_{i^{*}}\right)$, оставляя на месте $V_{i^{\prime}}$ и $V_{i^{\prime \prime}}$. Отсюда следует, что $\operatorname{dim}\left(\tilde{g}\left(V_{i^{*}}\right) \cap V_{i^{\prime \prime}}\right)=1$. Кроме того, с учетом $\tilde{h} \tilde{g} h_{i, j^{\prime}} \tilde{g}^{-1} \tilde{h}^{-1} \in \tilde{L}$ отсюда следует, что $\left\{\tilde{g}\left(V_{i}\right) \cap V_{i^{\prime}}, \tilde{g}\left(V_{i^{*}}\right) \cap V_{i^{\prime}}\right\}=X_{i^{\prime}}^{\xi}$ и $\left\{\tilde{g}\left(V_{i}\right) \cap V_{i^{\prime \prime}}, \tilde{g}\left(V_{i^{*}}\right) \cap V_{i^{\prime \prime}}\right\}=X_{i^{\prime \prime}}^{\xi^{\prime}}$ для некоторых $\xi, \xi^{\prime} \in\{-,+\}$. Остается доказать, что $\xi=\xi^{\prime}$. Элемент $h_{i^{\prime}, j^{\prime}} \in \tilde{L}$ инвертирует $V_{i^{\prime}}$ и централизует $V_{i^{\prime \prime}}$, а следовательно, меняет местами несингулярные прямые подпространства $\tilde{g}\left(V_{i}\right)$ пространства $V$, отличные от $\tilde{g}\left(V_{i}\right) \cap V_{i^{\prime}}$ и $\tilde{g}\left(V_{i}\right) \cap V_{i^{\prime \prime}}$. Но тогда $\tilde{g}\left(V_{i}\right) \cap V_{i^{\prime}} \in X_{i^{\prime}}^{\xi^{\prime \prime}}$ и $\tilde{g}\left(V_{i}\right) \cap V_{i^{\prime \prime}} \in X_{i^{\prime \prime}}^{\xi^{\prime \prime}}$ для некоторого $\xi^{\prime \prime} \in\{-,+\}$. Таким образом, $\xi=\xi^{\prime \prime}=\xi^{\prime}$. 
Так как $\operatorname{dim}\left(\tilde{g}\left(V_{1}\right) \cap V_{k}\right)=1=\operatorname{dim}\left(\tilde{g}\left(V_{1}\right) \cap V_{l}\right)$ (см. выше), то из доказанного утверждения следует существование такого $2 \leq i_{0} \leq t$, что $\operatorname{dim}\left(\tilde{g}\left(V_{i_{0}}\right) \cap V_{k}\right)=1=\operatorname{dim}\left(\tilde{g}\left(V_{i_{0}}\right) \cap V_{l}\right)$, причем $\left\{\tilde{g}\left(V_{1}\right) \cap V_{k}, \tilde{g}\left(V_{i_{0}}\right) \cap V_{k}\right\}=X_{k}^{\xi_{0}}$ и $\left\{\tilde{g}\left(V_{1}\right) \cap V_{l}, \tilde{g}\left(V_{i_{0}}\right) \cap V_{l}\right\}=X_{l}^{\xi_{0}}$ для некоторого $\xi_{0} \in\{-,+\}$.

Если $a \in O_{2}\left(\left(M_{1}, M_{2}\right)^{1}\right) \cap L$, то согласно (2.1) элемент а стабилизирует каждое из множеств прямых $X_{i}^{-}$и $X_{i}^{+}$для каждого $1 \leq i \leq t$. Покажем, что при этом для любого $\xi \in\{-,+\}$ элемент $а$ либо стабилизирует каждую прямую из $\cup_{1 \leq i \leq t} X_{i}^{\xi}$, либо сдвигает каждую прямую из $\cup_{1 \leq i \leq t} X_{i}^{\xi}$. Предположим, что, напротив, для некоторого $a \in O_{2}\left(\left(M_{1}, M_{2}\right)^{1}\right) \cap L$ и некоторого $\xi \in\{-,+\}$ найдутся $1 \leq i^{\prime}, i^{\prime \prime} \leq t, i^{\prime} \neq i^{\prime \prime}$ такие, что элемент a меняет местами прямые из $X_{i^{\prime}}^{\xi}$ и стабилизирует каждую прямую из $X_{i^{\prime \prime}}^{\xi}$. Так как группа $\widetilde{M}_{0}$ индуцирует на $\left\{V_{1}, \ldots, V_{t}\right\}$ симметрическую группу (см. выше), то, заменяя в случае необходимости элемент $a$ на элемент, сопряженный с ним в группе $M_{1}$, будем без потери общности предполагать, что $i^{\prime}=k$ и $i^{\prime \prime}=l$. Далее, заменяя в случае необходимости элемент $a$ на элемент $h a h^{-1}$, будем без потери общности предполагать, кроме того, что $\xi=\xi_{0}$. Но тогда $\tilde{a} \tilde{g}\left(V_{1}\right) \notin\left\{\tilde{g}\left(V_{1}\right), \ldots, \tilde{g}\left(V_{t}\right)\right\}$ и, следовательно, $a \notin M_{2}$, что противоречит $a \in O_{2}\left(\left(M_{1}, M_{2}\right)^{1}\right) \leq M_{2}$.

Теперь мы можем завершить рассмотрение случая $\varepsilon_{1}=+, q=5$ и случая $\varepsilon_{1}=-, q=3$. Для произвольных $1 \leq i_{1}, i_{2} \leq t, i_{1} \neq i_{2}$, элемент $g \bar{h}_{i_{1}, i_{2}} g^{-1} \in K_{2} \cap L \leq O_{2}\left(\left(M_{1}, M_{2}\right)^{1} \cap L\right.$ стабилизирует согласно (2.2) каждое из множеств прямых $X_{i}^{-}$и $X_{i}^{+}$для любого $1 \leq i \leq t$, причем, учитывая только что доказанное, для произвольного $\xi \in\{-,+\}$ либо стабилизирует каждую прямую из $\cup_{1 \leq i \leq t} X_{i}^{\xi}$, либо сдвигает каждую прямую из $\cup_{1 \leq i \leq t} X_{i}^{\xi}$. В частности, для произвольных $j^{\prime} \in\{2, \ldots, t\} \backslash\left\{i_{0}\right\}$ и $\xi^{\prime} \in\{-,+\} \backslash\left\{\xi_{0}\right\}$ ( $i_{0}$ и $\xi_{0}$ были определены выше) элемент $\tilde{g} h_{1, j^{\prime}} \tilde{g}^{-1}$, который инвертирует подпространство $\tilde{g}\left(V_{1}\right)$ пространства $V$ и централизует подпространство $\tilde{g}\left(V_{i_{0}}\right)$ пространства $V$, меняет местами прямые из $X_{i}^{\xi^{\prime}}$ для каждого $1 \leq i \leq t$. Мы покажем, однако, что последнее невозможно.

Пусть $i-$ некоторый элемент из $\{2, \ldots, t\} \backslash\left\{i_{0}\right\}$. Тогда (см. выше) справедливо одно из следующих утверждений:

1) $\tilde{g}\left(V_{i}\right) \in\left\{V_{1}, \ldots, V_{t}\right\}$;

2) найдутся $1 \leq i^{\prime}, i^{\prime \prime} \leq t, i^{\prime} \neq i^{\prime \prime}$, и $1 \leq i^{*} \leq t, i^{*} \neq i$, такие, что $\operatorname{dim}\left(\tilde{g}\left(V_{i}\right) \cap V_{i^{\prime}}\right)=$ $\operatorname{dim}\left(\tilde{g}\left(V_{i^{*}}\right) \cap V_{i^{\prime}}\right)=1=\operatorname{dim}\left(\tilde{g}\left(V_{i}\right) \cap V_{i^{\prime \prime}}\right)=\operatorname{dim}\left(\tilde{g}\left(V_{i^{*}}\right) \cap V_{i^{\prime \prime}}\right)$.

Если имеет место 1$)$, то, полагая выше $j^{\prime}=i$, получаем, что элемент $\tilde{g} h_{1, j^{\prime}} \tilde{g}^{-1}$ инвертирует подпространство $\tilde{g}\left(V_{i}\right) \in\left\{V_{1}, \ldots, V_{t}\right\}$ пространства $V$ и потому стабилизирует все прямые этого подпространства. Противоречие. Если имеет место 2), то, выбирая $j^{\prime} \in\{2, \ldots, t\} \backslash\left\{i_{0}, i, i^{*}\right\}$ (при этом мы пользуемся тем, что $L \neq O_{8}^{+}(q)$ и, следовательно, $t>4$ ), получаем, что элемент $\tilde{g} h_{1, j^{\prime}} \tilde{g}^{-1}$ централизует подпространства $\tilde{g}\left(V_{i}\right)$ и $\tilde{g}\left(V_{i^{*}}\right)$ пространства $V$, а потому с учетом $\left\langle\tilde{g}\left(V_{i}\right), \tilde{g}\left(V_{i^{*}}\right)\right\rangle=\left\langle V_{i^{\prime}}, V_{i^{\prime \prime}}\right\rangle$ централизует и подпространства $V_{i^{\prime}}$ и $V_{i^{\prime \prime}}$ пространства $V$. Противоречие.

Предположим, что q четно. Рассмотрим раздельно случаи $\varepsilon_{1}=+$ и $\varepsilon_{1}=-$.

Предположим, что $\varepsilon_{1}=+$. При этом предположении согласно [8, предложение 2.3.6] имеем $q \geq 8$. Из вида $\widetilde{M}_{1} \cap \Omega(V)$ (см. [8, табл. 2.5]) и $1 \neq K_{1} \cap L \unlhd M_{1}$ (см. лемму 1.2) следует существование для произвольных различных $1 \leq i_{1}, i_{2} \leq t$ элемента $h_{i_{1}, i_{2}}$ группы $\widetilde{K_{1}}$, который действует тождественно на каждом из подпространств $V_{i}, 1 \leq i \leq t, i_{1} \neq i \neq i_{2}$, векторного пространства $V$, не стабилизирует ни одну из несингулярных прямых подпространств $V_{i_{1}}$ и $V_{i_{2}}$ векторного пространства $V$ и умножает векторы сингулярных прямых подпространств $V_{i_{1}}$ и $V_{i_{2}}$ векторного пространства $V$ на неединичные элементы поля $F$ (различные, вообще говоря, для различных прямых). Заметим, что $h_{i_{1}, i_{2}}$ стабилизирует каждое подпространство $\tilde{g}\left(V_{i}\right)$, $1 \leq i \leq t$, векторного пространства $V$, поскольку $\bar{h}_{i_{1}, i_{2}} \in K_{1} \leq O_{p}\left(M_{2}\right) \leq C_{2}$ (см. (2.1)). Заменяя в случае необходимости вектор $w$ на вектор $w+h_{k, l}(w) \in \tilde{g}\left(V_{1}\right)$, будем в дальнейшем, не теряя общности, предполагать, что $w=w_{k}+w_{l}$. Выберем $1 \leq j \leq t$, отличное от $k$ и $l$. Если ни один из векторов $w_{k}, w_{l}$ не является сингулярным, то содержащиеся в $\tilde{g}\left(V_{1}\right)$ векторы $w, h_{k, j}(w)$ и $h_{l, j}(w)$ линейно независимы. Противоречие с $\operatorname{dim}\left(\tilde{g}\left(V_{1}\right)\right)=2$. Следовательно, $w_{k}$ или $w_{l}$ является сингулярным. Пусть, для определенности, вектор $w_{k}$ сингулярен. Тогда из 
$w+h_{k, j}(w) \in \tilde{g}\left(V_{1}\right)$ вытекает $w_{k} \in \tilde{g}\left(V_{1}\right)$, что, в свою очередь, влечет $w_{l}=w+w_{k} \in \tilde{g}\left(V_{1}\right)$. Таким образом, гиперболическая плоскость $\tilde{g}\left(V_{1}\right)$ содержит ортогональные векторы $w_{k} \in V_{k}$ и $w_{l} \in V_{l}$, причем вектор $w_{k}$ сингулярен, что невозможно. Полученное противоречие завершает рассмотрение случая $\varepsilon_{1}=+$ для четного $q$.

Предположим теперь, что $\varepsilon_{1}=-$. Из вида $\widetilde{M}_{1} \cap \Omega(V)$ (см. [8, табл. 2.5]) и $1 \neq K_{1} \cap L \unlhd M_{1}$ (см. лемму 2.2) следует существование для произвольных различных $1 \leq i_{1}, i_{2} \leq t$ элемента $h_{i_{1}, i_{2}}$ группы $\widetilde{K_{1}}$, который действует тождественно на каждом из подпространств $V_{i}$, $1 \leq i \leq t, i_{1} \neq i \neq i_{2}$, и не стабилизирует ни одну из прямых подпространств $V_{i_{1}}$ и $V_{i_{2}}$ векторного пространства $V$. Выбирая $1 \leq j \leq t$, отличное от $k$ и $l$, получаем теперь (рассматривая "проекции" векторов $w, h_{k, j}(w)$ и $h_{l, j}(w)$ на $V_{k}+V_{l}$ для разложения $V=V_{1}+\ldots+V_{t}$ и учитывая $K_{1} \leq C_{2}$, см. $\left.(2.2)\right)$, что содержащиеся в $\tilde{g}\left(V_{1}\right)$ векторы $w, h_{k, j}(w)$ и $h_{l, j}(w)$ линейно независимы. Противоречие с $\operatorname{dim}\left(\tilde{g}\left(V_{1}\right)\right)=2$ завершает рассмотрение случая $\varepsilon_{1}=-$ для четного $q$.

С л у ч а й $m=3$. Случай невозможен ввиду равенства $F^{*}\left(M_{1} \cap L\right)=O_{p}\left(M_{1} \cap L\right)$ и [8, предложение 2.3.6].

С л у ч а й $m=4$. В силу равенства $F^{*}\left(M_{1} \cap L\right)=O_{p}\left(M_{1} \cap L\right)$ и $[8$, предложение 2.3.6] для $\tilde{G}$ и $\widetilde{M}_{1}$ имеет место случай $\mathbf{O}^{\varepsilon}$, тип $G O_{4}^{\varepsilon_{1}}(q) 2 S_{t}$, из [8, табл. 2.4], где $\varepsilon=+$ (и, следовательно, $\operatorname{sgn}(V)=+), \varepsilon_{1}=+\left(\right.$ и, следовательно, $\operatorname{sgn}\left(V_{i}\right)=+$ для каждого $\left.1 \leq i \leq t\right)$ и $q=3, p=2$. При этом согласно [8, табл. 2.5] справедливы приводимые ниже утверждения 1)-3). (Предварительно напомним, что $\left|S O_{4}^{+}(3): \Omega_{4}^{+}(3)\right|=\left|G O_{4}^{+}(3): S O_{4}^{+}(3)\right|=\left|C G O_{4}^{+}(3): G O_{4}^{+}(3)\right|=2$, причем $\Omega_{4}^{+}(3) \cong F_{1} \circ F_{2}$, где $F_{1} \cong F_{2} \cong S L_{2}(3)$ и $Z\left(F_{1}\right)=Z\left(F_{2}\right), S O_{4}^{+}(3) \cong\left(F_{1} \circ F_{2}\right)\left\langle a_{1}\right\rangle$, где $a_{1}$ нормализует $F_{1}$ и $F_{2}$, причем $F_{1}\left\langle a_{1}\right\rangle \cong F_{2}\left\langle a_{1}\right\rangle \cong G L_{2}(3), G O_{4}^{+}(3)=S O_{4}^{+}(3)\left\langle a_{2}\right\rangle$, где $a_{2}$ при сопряжении меняет местами $F_{1}$ и $F_{2}$, и, наконец, $C G O_{4}^{+}(3)=G O_{4}^{+}(3)\left\langle a_{3}\right\rangle$, где $a_{3}$ нормализует подгруппу $S O_{4}^{+}(3)$ группы $G O_{4}^{+}(3)$ и $S O_{4}^{+}(3)\left\langle a_{3}\right\rangle \cong G L_{2}(3) \circ G L_{2}(3)$.)

1) Группа $\widetilde{M}_{1} \cap \Omega(V)$ имеет вид $\Omega_{4}^{+}(3)^{t} \cdot 2^{2(t-1)} . S_{t}$ (и при действии сопряжением индуцирует на множестве $\left\{V_{1}, \ldots, V_{t}\right\}$ симметрическую группу $\left.S_{t}\right)$.

2) Группа $\widetilde{C_{1}} \cap \Omega(V)$ имеет вид $\Omega_{4}^{+}(3)^{t} \cdot 2^{2(t-1)}$ и для каждого $1 \leq i \leq t$ индуцирует на $V_{i}$ группу $G O\left(V_{i}\right) \cong G O_{4}^{+}(3)$, причем содержит подгруппу, индуцирующую на $V_{i}$ группу $\Omega\left(V_{i}\right) \cong$ $\Omega_{4}^{+}(3)$ и действующую тривиально на каждом подпространстве из $\left\{V_{1}, \ldots, V_{t}\right\} \backslash\left\{V_{i}\right\}$.

3) Группа $\widetilde{C_{1}}$ для каждого $1 \leq i \leq t$ индуцирует на $V_{i}$ либо группу $G O\left(V_{i}\right) \cong G O_{4}^{+}(3)$, либо группу $C G O\left(V_{i}\right) \cong C G O_{4}^{+}(3)$.

Из 1) и 3) следует, что $O_{2}\left(\widetilde{M_{1}}\right)=O_{2}\left(\widetilde{C_{1}}\right) \cong O_{2}\left(\Omega_{4}^{+}(3)^{t}\right)$. С учетом 2) это влечет существование для произвольных различных $1 \leq i_{1}, i_{2} \leq t$ элемента $h_{i_{1}, i_{2}}$ группы $\widetilde{C_{1}}$, который действует тождественно на каждом из подпространств $V_{i}, 1 \leq i \leq t, i_{1} \neq i \neq i_{2}$, векторного пространства $V$ и инвертирует подпространства $V_{i_{1}}$ и $V_{i_{2}}$, причем элемент $\bar{h}_{i_{1}, i_{2}}$ принадлежит каждой неединичной нормальной подгруппе группы $M_{1} \cap \Omega(V)=M_{0}$. В частности, с учетом $K_{1} \cap L \neq 1 \neq K_{2} \cap L$ (см. лемму 2.2 ) для произвольных различных $1 \leq i_{1}, i_{2} \leq t$ имеем $h_{i_{1}, i_{2}} \in \widetilde{K_{1}}$ и $h_{i_{1}, i_{2}} \in \tilde{g}^{-1} \widetilde{K_{2}} \tilde{g}$.

Докажем справедливость для произвольных $\lambda \in\{-1,1\}$ и $1 \leq i \leq t$ равенства

$$
V_{i}=\left(V_{i} \cap \tilde{g}^{\lambda}\left(V_{1}\right)\right)+\left(V_{i} \cap \tilde{g}^{\lambda}\left(V_{2}\right)\right)+\ldots+\left(V_{i} \cap \tilde{g}^{\lambda}\left(V_{t}\right)\right) .
$$

Если $v=v_{1}+\ldots+v_{t}$, где $v \in V_{i}$ и $v_{1} \in \tilde{g}^{\lambda}\left(V_{1}\right), \ldots, v_{t} \in \tilde{g}^{\lambda}\left(V_{t}\right)$, то для произвольного $j \in\{1, \ldots, t\}$ и произвольных различных $j^{\prime}, j^{\prime \prime}$ из $\{1, \ldots, t\} \backslash\{j\}$ очевидным образом имеем

$$
v_{j}=\frac{1}{2}\left(\frac{1}{2}\left(v-\tilde{g}^{\lambda} h_{j, j^{\prime}} \tilde{g}^{-\lambda}(v)\right)-\tilde{g}^{\lambda} h_{j, j^{\prime \prime}} \tilde{g}^{-\lambda}\left(\frac{1}{2}\left(v-\tilde{g}^{\lambda} h_{j, j^{\prime}} \tilde{g}^{-\lambda}(v)\right)\right)\right),
$$

откуда в силу $\tilde{g}^{\lambda} h_{j, j^{\prime}} \tilde{g}^{-\lambda}, \tilde{g}^{\lambda} h_{j, j^{\prime \prime}} \tilde{g}^{-\lambda} \in O_{2}\left(\widetilde{M_{1}}\right) \leq \widetilde{C_{1}}$ (см. (2.1) и предыдущий абзац) заключаем, что $v_{j} \in V_{i}$. Это доказывает (2.4).

Согласно 2) существует подгруппа $A$ группы $\widetilde{C_{1}} \cap \Omega(V)$, индуцирующая на $V_{1}$ группу $\Omega\left(V_{1}\right)$ и действующая тривиально на каждом из подпространств $V_{2}, \ldots, V_{t}$ векторного пространства $V$. Ясно, что тогда $\tilde{g} A \tilde{g}^{-1}$ - подгруппа группы $\widetilde{C_{2}} \cap \Omega(V)$, индуцирующая на $\tilde{g}\left(V_{1}\right)$ группу $\Omega\left(\tilde{g}\left(V_{1}\right)\right) \cong \Omega_{4}^{+}(3)$ и действующая тривиально на каждом из подпространств $\tilde{g}\left(V_{2}\right), \ldots, \tilde{g}\left(V_{t}\right)$ 
пространства $V$. Пусть $j \in\{1, \ldots, t\} \backslash\{k, l\}$. Элемент $h_{k, j}$ группы $\widetilde{K_{1}} \leq \widetilde{C_{2}}$ (см. (2.2)) инвертирует ненулевое (в силу (2.4) для $\lambda=-1$ и $i=1$ ) подпространство $\tilde{g}\left(V_{1}\right) \cap V_{k}$ и централизует ненулевое (в силу (2.4) для $\lambda=-1$ и $i=1$ ) подпространство $\tilde{g}\left(V_{1}\right) \cap V_{l}$ пространства $\tilde{g}\left(V_{1}\right)$. Поэтому подгруппа $B:=\left\langle x h_{k, j} x^{-1} \mid x \in \tilde{g} A \tilde{g}^{-1}\right\rangle$ группы $\widetilde{C_{2}}$ индуцирует на $\tilde{g}\left(V_{1}\right)$ группу, содержащую $O_{2}\left(\Omega\left(\tilde{g}\left(V_{1}\right)\right)\right)$, и, в частности, действует неприводимо на $\tilde{g}\left(V_{1}\right)$. Следовательно, учитывая, что $0 \neq \tilde{g}\left(V_{1}\right) \cap V_{k} \neq \tilde{g}\left(V_{1}\right)$, для некоторого $b \in B$ имеем $b\left(V_{k}\right) \neq V_{k}$. Но из $h_{k, j} \in \widetilde{K_{1}}, K_{1} \leq\left(M_{2}, M_{1}\right)^{1} \unlhd M_{2}$ и $\tilde{g} A \tilde{g}^{-1} \leq \widetilde{M}_{2}$ следует, что $B \leq \widehat{\left(M_{2}, M_{1}\right)^{1}} \leq \widetilde{M_{1}}$, и потому $B$ стабилизирует разложение $V=V_{1}+\ldots+V_{t}$. Следовательно, $b\left(V_{k}\right)=V_{k^{\prime}}$ для некоторого $k^{\prime} \in\{1, \ldots, t\} \backslash\{k\}$. Далее, согласно (2.4) имеем

$$
\begin{gathered}
V_{k}=\left(V_{k} \cap \tilde{g}\left(V_{1}\right)\right)+\left(V_{k} \cap \tilde{g}\left(V_{2}\right)\right)+\ldots+\left(V_{k} \cap \tilde{g}\left(V_{t}\right)\right), \\
V_{k^{\prime}}=\left(V_{k^{\prime}} \cap \tilde{g}\left(V_{1}\right)\right)+\left(V_{k^{\prime}} \cap \tilde{g}\left(V_{2}\right)\right)+\ldots+\left(V_{k^{\prime}} \cap \tilde{g}\left(V_{t}\right)\right) .
\end{gathered}
$$

Для произвольного $i \in\{2, \ldots, t\}$ группа $B$ стабилизирует каждое одномерное подпространство векторного пространства $V_{k} \cap \tilde{g}\left(V_{i}\right)$. Действительно, для любого $x \in \tilde{g} A \tilde{g}^{-1}$ элемент $x h_{k, j} x^{-1}$ стабилизирует каждое одномерное подпространство векторного пространства $V_{k} \cap \tilde{g}\left(V_{i}\right)$, поскольку $x$ действует тривиально на $\tilde{g}\left(V_{i}\right)$, а $h_{k, j}$ инвертирует $V_{k}$. Но тогда с учетом $B \leq \widetilde{C_{2}}$ для произвольного $i \in\{2, \ldots, t\}$ имеем

$$
V_{k} \cap \tilde{g}\left(V_{i}\right)=b\left(V_{k} \cap \tilde{g}\left(V_{i}\right)\right)=b\left(V_{k}\right) \cap b\left(\tilde{g}\left(V_{i}\right)\right)=V_{k^{\prime}} \cap \tilde{g}\left(V_{i}\right),
$$

что в силу $V_{k} \cap V_{k^{\prime}}=\{0\}$ влечет $V_{k} \cap \tilde{g}\left(V_{i}\right)=\{0\}$. Таким образом,

$$
V_{k}=\left(V_{k} \cap \tilde{g}\left(V_{1}\right)\right)+\left(V_{k} \cap \tilde{g}\left(V_{2}\right)\right)+\ldots+\left(V_{k} \cap \tilde{g}\left(V_{t}\right)\right)=V_{k} \cap \tilde{g}\left(V_{1}\right)
$$

и, следовательно, $V_{k}=\tilde{g}\left(V_{1}\right)$, что противоречит выбору $V_{1}$ с условием $\tilde{g}\left(V_{1}\right) \notin\left\{V_{1}, \ldots, V_{t}\right\}$. Полученное противоречие завершает рассмотрение случая $m=4$.

Справедливость теоремы в рассматриваемом в данном разделе случае следует из лемм 2.1-2.4.

\section{3. Доказательство теоремы в случае, когда группа $L$ изоморфна $O_{8}^{+}(q)$}

В дальнейшем доказательстве теоремы будем предполагать, что группа $L$ изоморфна $O_{8}^{+}(q)$, где $q=r^{e}$. Группа $O u t(L)$ имеет следующее копредставление (см. [8, разд. 1.7.1]): $\left\langle\tau, \gamma, \varphi \mid \tau^{3}=\gamma^{2}=(\gamma \tau)^{2}=\varphi^{e}=[\tau, \varphi]=[\gamma, \varphi]=1\right\rangle$ при четном $q$ и $\left\langle\tau, \gamma, \varphi, \delta, \delta^{\prime}\right| \tau^{3}=\gamma^{2}=$ $\left.(\gamma \tau)^{2}=\delta^{2}=\delta^{\prime 2}=1, \delta^{\tau}=\delta^{\prime},\left(\delta^{\prime}\right)^{\tau}=\delta \delta^{\prime},(\delta \gamma)^{2}=\delta^{\prime}, \varphi^{e}=[\delta, \varphi]=[\tau, \varphi]=[\gamma, \varphi]=1\right\rangle$ при

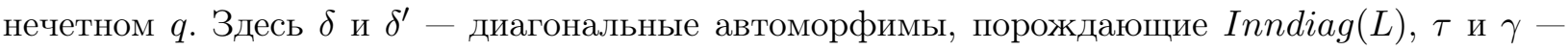
графовые автоморфизмы и $\varphi-$ полевой автоморфим порядка $e$ группы $L$. Группа $\left\langle\delta^{\prime}\right\rangle$ расширяет $\operatorname{Inn}(L)=O(V)$ до $P S O(V),\left\langle\delta^{\prime}, \gamma\right\rangle$ расширяет $O(V)$ до $P G O(V),\left\langle\delta^{\prime}, \gamma, \delta\right\rangle$ расширяет $O(V)$ до $P C G O(V),\left\langle\delta^{\prime}, \gamma, \delta, \varphi\right\rangle$ расширяет $O(V)$ до $P \Gamma O(V)$. Обозначим через через $c$ число $L$-классов подгрупп, сопряженных с $M_{0}$ в $A u t(L)$, а через $S$ стабилизатор $L$-класса подгрупп с представителем $M_{0}$ относительно действия группы $O u t(L)$.

Ввиду равенства $F^{*}\left(M_{0}\right)=O_{p}\left(M_{0}\right),[8$, табл. 8.50] и [6] выполняется один из следующих случаев, которые упорядочены как в [8, табл. 8.50]:

(i) $q=2, M_{0} \cong \Omega_{2}^{-}(3) \times G U_{3}(2) .2 \cong \mathbb{Z}_{3} \times\left(3_{+}^{1+2}: 2 S_{4}\right), M \cong 3_{+}^{1+4}: 2 S_{4}$ или $3_{+}^{1+4} \cdot\left(2 S_{4} \times \mathbb{Z}_{2}\right)$, $S=\operatorname{Out}(L)$

(ii) $q=r \equiv \pm 3(\bmod 8), \widetilde{M}_{0} \cap \Omega(V) \cong 2^{7} . A_{8}, M_{0} \cong 2^{6} . A_{8}, c=2, S=\left\langle\gamma, \delta^{\prime}\right\rangle, G / L \not \leq\left\langle\gamma \delta^{\prime}\right\rangle$;

(iii) $q=r \equiv \pm 3(\bmod 8), \widetilde{M}_{0} \cap \Omega(V) \cong 2_{+}^{1+6} . A_{8}, M_{0} \cong 2^{6} . A_{8}, c=4, S=\left\langle\gamma, \delta^{\prime}\right\rangle$;

(iv) $q=r \equiv \pm 1(\bmod 8), \widetilde{M}_{0} \cap \Omega(V) \cong 2^{7} . S_{8}, c=4, S=\langle\gamma\rangle$;

(v) $q=r \equiv \pm 1(\bmod 8), \widetilde{M}_{0} \cap \Omega(V) \cong 2_{+}^{1+6} . S_{8}, c=8, S=\langle\gamma\rangle$ 
(vi) $q=r \geq 3, \widetilde{M}_{0} \cap \Omega(V) \cong 2^{4} \cdot 2^{6} \cdot L_{3}(2), M_{0} \cong 2^{3} \cdot 2^{6} \cdot L_{3}(2), c=4, G / L \cong \mathbb{Z}_{3}$ или $S_{3}$, $S=\langle\gamma, \tau\rangle$

(vii) $q \geq 5, \widetilde{M}_{0} \cap \Omega(V) \cong \Omega_{2}^{+}(q)^{4} \cdot[2(2, q-1)]^{3} \cdot S_{4}, c=1, G / L$ не содержится в подгруппе из $\operatorname{Out}(L)$, сопряженной с $\left\langle\gamma, \delta^{\prime}\right\rangle$ или $\langle\tau, \gamma\rangle$ при $q=5$;

(viii) $\widetilde{M}_{0} \cap \Omega(V) \cong \Omega_{2}^{-}(q)^{4} \cdot[2(2, q-1)]^{3} \cdot S_{4}, c=1, G / L$ не содержится в подгруппе из $O u t(L)$, сопряженной с $\left\langle\tau, \delta^{\prime}\right\rangle$ или $\langle\tau, \gamma\rangle$ при $q=3$;

(ix) $q=3, \widetilde{M}_{0} \cap \Omega(V) \cong \Omega_{4}^{+}(3)^{2} \cdot[4] . S_{2}, M_{0} \cong 2 \cdot\left(\left(A_{4}\right.\right.$ 乙2) 22$) \cong\left(S L_{2}(3) \circ S L_{2}(3)\right) \cdot D_{8}, c=1$;

(x) $\widetilde{M}_{0} \cap \Omega(V) \cong\left(D_{2\left(q^{2}+1\right) /(2, q-1)}\right)^{2} \cdot[2(2, q-1)] \cdot S_{2}, c=1$.

Лемма 3.1. Подгруппы $K_{1}$ и $K_{2}$ имеют нетривиальные пересечения с $L$.

Д о к а з а т е л ь с т в о. Предположим противное. Тогда ввиду леммы 1.2 число $q$ нечетно, $L E=\operatorname{Inndiag}(L)<G$ для некоторой четверной подгруппы $E, M=N_{L}(E)$ и, следовательно, выполняется один из случаев (vii)-(x). Если подгруппа $M_{0}=C_{L}(E)$ максимальна в $L$, то $M_{0}=C_{L}(x)$ для любой инволюции $x$ из $E$, что противоречит $[13,4.5 .1]$. Поэтому выполняется либо случай (vii) с $q=5$, либо случай (viii) с $q=3$. Но тогда $E$ централизует силовскую 2-подгруппу в $M_{0}$, которая является силовской 2-подгруппой в $L$, и ввиду [14] имеем $M_{0}<Y<X<L$, где $Y \cong 2^{6}: A_{8}$ и $X \cong \Omega_{8}^{+}(2)$. Отсюда следует, что подгруппа $E$ нормализует $O_{2}(Y)$ в противоречии с [14; 8 , табл. 8.50].

Рассмотрим далее каждый из случаев (i)-(х).

Пусть выполняется случай (i). Рассуждая, как в случае (iv) доказательства леммы 2.2 из [4, ч. III], приходим к противоречию.

Пусть выполняется один из случаев (ii)-(v). Тогда $M_{i} \cap L$ неприводимо действует на $O_{2}\left(M_{i} \cap L\right)$ для $i \in\{1,2\}$ и, следовательно, $T_{i}=O_{2}\left(M_{i} \cap L\right)$ для $i \in\{1,2\}$. Но $T_{2} \leq O_{2}\left(M_{1} \cap L\right)$ и, следовательно, $T_{1}=T_{2}$, что невозможно.

Пусть выполняется случай (ix). Тогда $O_{2}\left(M_{i} \cap L\right) \cong 2_{+}^{1+16}$ и $M_{i} \cap L$ неприводимо действует на $O_{2}\left(M_{i} \cap L\right) / Z\left(O_{2}\left(M_{i} \cap L\right)\right)$ для $i \in\{1,2\}$. Поскольку $T_{i} \triangleleft M_{i}$ для $i \in\{1,2\}$, отсюда следует, что $T_{i}=Z\left(O_{2}\left(M_{i} \cap L\right)\right)$ для $i \in\{1,2\}$. Поскольку $T_{1}<T_{1} T_{2} \leq R_{1} \cap L$, имеем $R_{1} \cap L=O_{2}\left(M_{0}\right)$. Но тогда $T_{2} \triangleleft O_{2}\left(M_{0}\right)$ и, следовательно, $T_{1}=T_{2}$, что невозможно.

Пусть выполняется случай (х). Используя [11], получаем, что $M_{0}-p$-замкнутая группа, $p>2$ и подгруппа $O_{p}\left(M_{0}\right)$ абелева. Поскольку $1 \neq T_{2} \leq O_{p}\left(M_{0}\right)$, имеем $O_{p}\left(M_{0}\right) \leq N_{L}\left(T_{2}\right)=$ $M_{2} \cap L$. Но подгруппа $M_{2} \cap L$, равная $g M_{0} g^{-1}$, также $p$-замкнута, поэтому $O_{p}\left(M_{0}\right)=O_{p}\left(M_{2} \cap L\right)$, что невозможно.

Пусть выполняется случай (vi). Тогда из [14] и [8, табл. 8.50] видно, что $M_{0}<Y<X<L$, где $Y \cong 2^{6}: A_{8}$ и $X \cong \Omega_{8}^{+}(2)$. Можно считать, что $X=\Phi(k)$, где $\Phi$ - корневая система типа $D_{4}$ и $k=G F(2)$. Ввиду [1, табл. IV] $\Phi$ имеет фундаментальную систему корней $\Pi=\left\{\alpha_{1}, \alpha_{2}, \alpha_{3}, \alpha_{4}\right\}$ со схемой Дынкина

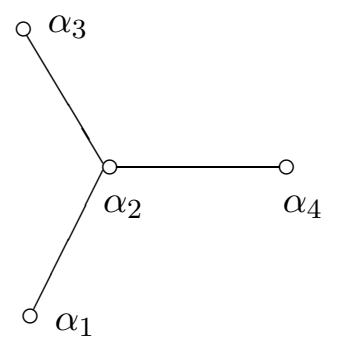

и множеством положительных корней $\Phi^{+}=\left\{\alpha_{1}, \alpha_{2}, \alpha_{3}, \alpha_{4}, \alpha_{1}+\alpha_{2}, \alpha_{3}+\alpha_{2}, \alpha_{4}+\alpha_{2}, \alpha_{1}+\right.$ $\left.\alpha_{2}+\alpha_{3}, \alpha_{1}+\alpha_{2}+\alpha_{4}, \alpha_{2}+\alpha_{3}+\alpha_{4}, \alpha_{1}+\alpha_{2}+\alpha_{3}+\alpha_{4}, \alpha_{1}+2 \alpha_{2}+\alpha_{3}+\alpha_{4}\right\}$. Пусть $X_{\alpha}=\left\langle x_{\alpha}(1)\right\rangle-$ корневая подгруппа группы $X$, соответствующая корню $\alpha \in \Phi$. Положим для краткости $x_{\alpha}=x_{\alpha}(1)$. Пусть $U=\prod_{\alpha \in \Phi^{+}} X_{\alpha}-$ унипотентная подгруппа группы $X$. Используя коммутаторную формулу Шевалле (см. [9, теорема 5.2.2]), легко проверяется, что все нетривиальные коммутаторы пар порождающих $x_{\alpha}, \alpha \in \Phi^{+}$, группы $U$ 
(с точностью до перестановки элементов пары) исчерпываются следующими: $\left[x_{\alpha_{1}}, x_{\alpha_{2}}\right]=$ $x_{\alpha_{1}+\alpha_{2}}, \quad\left[x_{\alpha_{1}}, x_{\alpha_{2}+\alpha_{3}}\right]=\left[x_{\alpha_{3}}, \quad x_{\alpha_{1}+\alpha_{2}}\right]=x_{\alpha_{1}+\alpha_{2}+\alpha_{3}},\left[x_{\alpha_{1}}, x_{\alpha_{2}+\alpha_{4}}\right]=\left[x_{\alpha_{4}}, x_{\alpha_{1}+\alpha_{2}}\right]=$ $x_{\alpha_{1}+\alpha_{2}+\alpha_{4}}, \quad\left[x_{\alpha_{1}}, x_{\alpha_{2}+\alpha_{3}+\alpha_{4}}\right]=\left[x_{\alpha_{3}}, x_{\alpha_{1}+\alpha_{2}+\alpha_{4}}\right]=\left[x_{\alpha_{4}}, x_{\alpha_{1}+\alpha_{2}+\alpha_{3}}\right]=x_{\alpha_{1}+\alpha_{2}+\alpha_{3}+\alpha_{4}}$, $\left[x_{\alpha_{3}}, x_{\alpha_{2}}\right]=x_{\alpha_{2}+\alpha_{3}}, \quad\left[x_{\alpha_{4}}, x_{\alpha_{2}}\right]=x_{\alpha_{2}+\alpha_{4}},\left[x_{\alpha_{3}}, x_{\alpha_{1}+\alpha_{2}}\right]=x_{\alpha_{1}+\alpha_{2}+\alpha_{3}},\left[x_{\alpha_{2}}, x_{\alpha_{1}+\alpha_{2}+\alpha_{3}+\alpha_{4}}\right]=$ $\left[x_{\alpha_{1}+\alpha_{2}}, x_{\alpha_{2}+\alpha_{3}+\alpha_{4}}\right]=\left[x_{\alpha_{2}+\alpha_{3}}, x_{\alpha_{1}+\alpha_{2}+\alpha_{4}}\right]=\left[x_{\alpha_{2}+\alpha_{4}}, x_{\alpha_{1}+\alpha_{2}+\alpha_{3}}\right]=x_{\alpha_{1}+2 \alpha_{2}+\alpha_{3}+\alpha_{4}}$.

Поскольку $\left|M_{0}\right|_{2}=|X|_{2}$, можно считать, что $U<M_{0}$. Ясно, что $U$ является подгруппой Бореля, а $M_{0}$ - параболической подгруппой в $X$. Положим $P_{i}=\left\langle U, X_{-\alpha} \mid \alpha \in \Pi \backslash\left\{\alpha_{i}\right\}\right\rangle$ и $\Psi_{i}=\left\{\sum_{j=1}^{4} k_{j} \alpha_{j} \in \Phi^{+} \mid k_{i}>0\right\}$ для $i \in\{1,3,4\}$. Ввиду [9, разд. 8.3] $P_{1}, P_{2}, P_{3}, P_{4}-$ все параболические максимальные подгруппы в $X$, содержащие $U$, и для $i \in\{1,3,4\}$ имеем $P_{i}=U_{i} L_{i}$, где $U_{i}=\prod_{\alpha \in \Psi_{i}} X_{\alpha}$ - унипотентный радикал и $L_{i}=\left\langle X_{ \pm \alpha} \mid \alpha \in \Pi \backslash\left\{\alpha_{i}\right\}\right\rangle-$ дополнение Леви в $P_{i}$, причем $L_{i} \cong G L_{4}(2) \cong A_{8}$ для $i \in\{1,3,4\}$. Из вышеприведенных коммутаторных соотношений следует, что $U_{i} \cong 2^{6}$ для $i \in\{1,3,4\}$.

Рассмотрим теперь параболическую подгруппу $P_{0}=P_{3} \cap P_{4}$ в $X$. Имеем $P_{0}=U_{0} L_{0}$, где $U_{0}=U_{3} U_{4}-$ унипотентный радикал и $L_{0}=\left\langle X_{ \pm \alpha_{1}}, X_{ \pm \alpha_{2}}\right\rangle \cong L_{3}(2)-$ дополнение Леви в $P_{0}$. Из вышеприведенных коммутаторных соотношений следует, что

$$
U_{3} \cap U_{4}=Z\left(U_{0}\right)=\Phi\left(U_{0}\right)=\left[U_{0}, U_{0}\right]=X_{\alpha_{2}+\alpha_{3}+\alpha_{4}} \times X_{\alpha_{1}+\alpha_{2}+\alpha_{3}+\alpha_{4}} \times X_{\alpha_{1}+2 \alpha_{2}+\alpha_{3}+\alpha_{4}} .
$$

Отсюда $U_{0} / Z\left(U_{0}\right)=\left(U_{3} / Z\left(U_{0}\right)\right) \times\left(U_{4} / Z\left(U_{0}\right)\right)$ и $L_{0}$ точно и неприводимо действует на $Z\left(U_{0}\right)$, $U_{3} / Z\left(U_{0}\right)$ и $U_{4} / Z\left(U_{0}\right)$.

В рассматриваемом нами случае (vi) можно считать, что $M_{0}=P_{0}$. Ввиду [14] и условия (vi) имеем $M / O_{2}\left(M_{0}\right) \cong L_{3}(2) \times \mathbb{Z}_{3}$ или $L_{3}(2) \times S_{3}$, следовательно, $O_{2}(M)=O_{2}\left(M_{0}\right)$ и $M$ действует неприводимо на $U_{0} / Z\left(U_{0}\right)$. Ясно, что $K_{1}=Z\left(U_{0}\right), M / C_{M}\left(K_{1}\right) \cong L_{3}(2)$ и $C_{M_{0}}\left(K_{1}\right)=U_{0}$. Поэтому $\left[M_{0}, C_{M}\left(K_{1}\right)\right] \leq M_{0} \cap C_{M}\left(K_{1}\right)=C_{M_{0}}\left(K_{1}\right)=U_{0}$ и, следовательно, $C_{M}\left(K_{1}\right)$ содержит элемент $\tau^{*}$ порядка 3 , нормализующий подгруппу $U$. При этом можно считать, что $\tau^{*} L=\tau$.

Обозначим через $A b(U)$ множество всех абелевых подгрупп наибольшего порядка из $U$. Ввиду [7, теорема 3.1, лемма 3.10] множество $A b(U)$ состоит из семи элементарных абелевых подгрупп порядка 64 и содержит нормальные в $U$ подгруппы $U_{1}, U_{3}, U_{4}$. Подгруппа $\left\langle\tau^{*}\right\rangle$ действует на множестве $A b(U)$, не фиксируя ввиду [8, табл. 8.50] ни одной из подгрупп $U_{1}, U_{3}$, $U_{4}$. Пусть $O_{i}$ обозначает $\left\langle\tau^{*}\right\rangle$-орбиту на $A b(U)$, содержащую $U_{i}$, для $i \in\{1,3,4\}$. Подгруппы из $O_{3} \cup O_{4}$ содержатся в $U_{0}$, а подгруппы из $O_{1}$ не содержатся в $U_{0}$, так как $U_{0} \triangleleft M_{1},\left|U_{0}\right|=2^{9}$ и $\left|U_{1} U_{3} U_{4}\right|=2^{11}$. Поэтому орбиты $O_{1}$ и $O_{3}$ различны и, следовательно, множество $O_{1} \cup O_{3}$ состоит из шести нормальных в $U$ подгрупп. Но тогда и оставшаяся подгруппа из множества $A b(U) \backslash\left(O_{1} \cup O_{3}\right)$ также нормальна в $U$. Таким образом, каждая абелева подгруппа порядка 64 из $U$ нормальна в $U$.

Поскольку $1 \neq K_{i}<O_{2}\left(M_{i}\right)$ для $i \in\{1,2\}$, имеем $K_{i}=Z\left(O_{2}\left(M_{i}\right)\right)$ для $i \in\{1,2\}$, так что $K_{2}=g K_{1} g^{-1}$ и, следовательно, $O_{2}\left(M_{2}\right)=C_{L}\left(K_{2}\right)=g C_{L}\left(K_{1}\right) g^{-1}=g O_{2}\left(M_{1}\right) g^{-1}=g U_{0} g^{-1}$.

Поскольку $K_{1}<K_{1} K_{2}<R_{1} \leq O_{2}\left(M_{0}\right)$, имеем $R_{1}=O_{2}\left(M_{0}\right)$ и, следовательно, $K_{2} \triangleleft U_{0}$. Поэтому $U_{0} g U_{0} g^{-1}$ является 2-подгруппой в $M_{0}$. Подправляя элемент $g$ на подходящий множитель из $M_{0}$, мы можем считать, что $U_{0} g U_{0} g^{-1} \leq U$. Но тогда $g U_{0} g^{-1}=g U_{3} g^{-1} g U_{4} g^{-1}$, где подгруппы $g U_{3} g^{-1}$ и $g U_{4} g^{-1}$ нормальны в $U$, поэтому подгруппа $g U_{0} g^{-1}$ нормальна в $U$. Отсюда следует, что $U$ и $g^{-1} U g$ являются силовскими 2-подгруппами в $M_{0}$. Поэтому существует элемент $h \in M_{0}$ такой, что $h^{-1}\left(g^{-1} U g\right) h=U$. Поскольку элемент $g$ можно заменить на элемент $g h$, мы можем считать, что $g$ нормализует $U$ и, в частности, $U \leq M_{1} \cap M_{2}$.

Используя вышеприведенные коммутаторные соотношения, легко видеть, что

$$
[U, U]=\Phi(U)=\left\langle X_{\alpha} \mid \alpha \in \Phi^{+} \backslash\left\{\alpha_{1}, \alpha_{2}, \alpha_{3}, \alpha_{4}\right\}\right\rangle .
$$

Кроме того, в силу [7, теорема 6.1(j)] подгруппа Томпсона $J(U)=\langle A \mid A \in A b(U)\rangle$ группы $U$ имеет вид $J(U)=[U, U]\left(X_{\alpha_{1}} \times X_{\alpha_{3}} \times X_{\alpha_{4}}\right)$. Ясно, что $Z(U)=X_{\alpha_{1}+2 \alpha_{2}+\alpha_{3} \alpha_{4}}$ и, следовательно, $C_{M_{0}}(Z(U))=J(U)\left\langle X_{ \pm \alpha_{2}}\right\rangle \cong J(U): S_{3}$ и $C_{M_{1}}(Z(U)) \cong J(U):\left(S_{3} \times 3\right)$ или $J(U):\left(S_{3} \times S_{3}\right)$. Поскольку $g$ нормализует $U$, имеем $U \leq M_{1} \cap M_{2} \cap L \leq C_{M_{0}}(Z(U))$. 
Предположим, что $q \equiv \pm 3(\bmod 8)$, т. е. $U-$ силовская 2 -подгруппа в $L$. Ввиду $[2$, следствие] подгруппа $U$ самонормализуема в $L$. Поэтому по теореме Бернсайда [12, теорема 7.1.1] каждая абелева подгруппа порядка 64 из $U$ слабо замкнута в $U$ относительно $L$. В частности, подгруппа $U_{0}=U_{3} U_{4}$ слабо замкнута в $U$ относительно $L$. Ввиду включения $U_{0} \leq M_{1} \cap g M_{2} g^{-1}$ отсюда следует, что элемент $g$ нормализует $U_{0}$ и, следовательно, принадлежит $M_{0}$; противоречие.

Итак, $q \equiv \pm 1(\bmod 8)$ и, следовательно, подгруппа $U$ строго содержится в некоторой силовской 2-подгруппе $T$ из $L$. Поэтому $U<N_{T}(U)$. Рассмотрим подгруппу $N=N_{L\left\langle\tau^{*}\right\rangle}(U)$ и положим $\hat{N}=N / \Phi(U)$. Тогда $\hat{U}=\widehat{X_{\alpha_{1}}} \times \widehat{X_{\alpha_{2}}} \times \widehat{X_{\alpha_{3}}} \times \widehat{X_{\alpha_{4}}}$ и, следовательно, можно рассматривать $\hat{U}$ как 4-мерное пространство над полем $G F(2)$. Поскольку $N_{M_{0}}(U)=U$, имеем $N_{N}\left(U_{0}\right)=U\left\langle\tau^{*}\right\rangle$ и $C_{\hat{N}}\left(\widehat{U_{0}}\right)=\hat{U}$. Таким образом, группа $N / U$ действует на $\hat{U}$ точно, оставляя 3 -мерное подпространство $\widehat{J(U)}$ инвариантным, и стабилизатор в $N / U$ гиперплоскости $\widehat{U_{0}}$ из $\widehat{J(U)}$ равен $U\left\langle\tau^{*}\right\rangle / U$. Поскольку $U<N_{T}(U)$, порядок группы $N / U$ делится на 6 . Теперь подгрупповое строение группы $G L_{4}(2) \cong A_{8}$ (см. [6]) показывает, что $N / U$ - циклическая группа порядка 6 и, в частности, $N_{L}(U)=T$ и $|T: U|=2$. Поскольку $g \in N$, можно считать, что элемент $g$ централизует $\tau^{*}$, поэтому $C_{M_{1}}(Z(U))=C_{M_{2}}(Z(U))=M_{1} \cap M_{2}$. Отсюда ввиду теоремы из [16], примененной к амальгаме $\left\{M_{1}, M_{2}, M_{1} \cap M_{2}\right\}$, получаем, что $M_{1} \cap M_{2}=U$.

Возьмем теперь любой элемент $t$ из $N_{T}(U) \backslash U$, централизующий $\tau^{*}$. Тогда $t^{2} \in U$, $t M t^{-1} \neq M$ и $U \leq M \cap t M t^{-1}$. Имеем $O_{2}\left(\left(M, t M t^{-1}\right)^{1}\right)=U_{0}$ и $O_{2}\left(\left(t M t^{-1}, M\right)^{1}\right)=t U_{0} t^{-1} \neq U_{0}$, причем $U_{0}$ и $t U_{0} t^{-1}$ являются нормальными подгруппами индекса 8 в $U$. Подгруппа $J(U)$ имеет индекс 2 в $U$ и содержит $U_{0} t U_{0} t^{-1}$. Группа $U_{0} t U_{0} t^{-1} / U_{0} \cong U_{0} /\left(U_{0} \cap t U_{0} t^{-1}\right.$ содержится в группе $J(U) / U_{0}$, имеющей порядок 4. Поэтому $\left[U_{0}, U_{0}\right] \leq U_{0} \cap t U_{0} t^{-1}$. Аналогично $\left[t U_{0} t^{-1}, t U_{0} t^{-1}\right] \leq U_{0} \cap t U_{0} t^{-1}$. Поэтому $\left(M, t M t^{-1}\right)^{2}=\left[U_{0}, U_{0}\right]=Z\left(U_{0}\right)$ и $\left(t M t^{-1}, M\right)^{1}=$ $\left[t U_{0} t^{-1}, t U_{0} t^{-1}\right]=Z\left(t U_{0} t^{-1}\right)$.

Итак, в случае (vi) для $G$ имеется единственная с точностью до эквивалентности тройка $\left(G, M_{1}, M_{2}\right)$ из П, и для нее выполняется п. (а) теоремы.

Таким образом, можно считать, что выполняется один из случаев (vii), (viii). Рассмотрение этих случаев во многом аналогично рассмотрению случая $m=2$ при доказательстве леммы 2.4. Особенно это справедливо для четного $q$, и наше рассмотрение случаев (vii) и (viii) при четном $q$ состоит в указании немногочисленных изменений, которые необходимо внести для его получения в рассмотрение случая $m=2$ при четном $q$ из доказательства леммы 2.4. Однако при нечетном $q$ многочисленные (и, по большей части, усложняющие доказательство) изменения, которые необходимо внести в рассмотрение случая $m=2$ из доказательства леммы 2.4, чтобы адаптировать его к случаям (vii) и (viii), делают целесообразным изложить рассмотрение случаев (vii) и (viii) при нечетном $q$ замкнутым образом.

Подгруппа $\widetilde{M}_{0}$ есть стабилизатор в $\tilde{L}$ ортогонального разложения $V=V_{1}+V_{2}+V_{3}+V_{4}$ пространства $V$ на изометричные невырожденные 2-мерные подпространства $V_{1}, V_{2}, V_{3}, V_{4}$ типа $\varepsilon_{1}$. Строение группы $\widetilde{M}_{0}$ дается в (vii) при $\varepsilon_{1}=+$ и дается в (viii) при $\varepsilon_{1}=-$. Из этого строения следует, что группа $\widetilde{M}_{0}$ индуцирует на множестве $\left\{V_{1}, V_{2}, V_{3}, V_{4}\right\}$ симметрическую группу подстановок $S_{4}$. Напомним, что согласно лемме 3.1 подгруппы $K_{1} \cap L$ и $K_{2} \cap L$ нетривиальны.

Если $\varepsilon_{1}=+$, то, учитывая $O(V)=[P G O(V), P G O(V)]$, замечаем, что для каждого $1 \leq i \leq 4$ стабилизатор $V_{i}$ в группе $\overline{M_{1} \cap O(V)}=\widetilde{M}_{0}$, индуцирует на множестве одномерных подпространств пространства $V_{i}$ группу $P G O\left(V_{i}\right) \cong P G O_{2}^{+}(q) \cong D_{(2, q)(q-1)}$. Более того, для произвольных различных $1 \leq i_{1}, i_{2} \leq 4$ в $M_{0}$ имеется подгруппа $R_{i_{1}, i_{2}}$, стабилизирующая все одномерные подпространства пространств $V_{j}, 1 \leq j \leq 4, i_{1} \neq j \neq i_{2}$, и индуцирующая на множестве одномерных подпространств пространства $V_{i_{1}}$ группу $P G O\left(V_{i_{1}}\right)$, а на множестве одномерных подпространств пространства $V_{i_{2}}$ группу $P G O\left(V_{i_{2}}\right)$. Отметим, что группа $P G O\left(V_{i}\right), 1 \leq i \leq 4$, действует транзитивно на множестве (из $q-1$ ) несингулярных прямых $V_{i}$ в случае четного $q$, а в случае нечетного $q$ имеет на этом множестве в точности две орбиты, длиной $(q-1) / 2$ каждая, являющиеся множеством несингулярных прямых $V_{i}$, значе- 
ния квадратичной формы на векторах которых являются квадратами в поле $F$, и множеством прямых $V_{i}$, значения квадратичной формы на ненулевых векторах которых не являются квадратами в поле $F$.

Если $\varepsilon_{1}=-$, то для каждого $1 \leq i \leq 4$ подпространство $V_{i}$ пространства $V$ не содержит ненулевых сингулярных векторов и, поскольку $O(V)=[P G O(V), P G O(V)]$, стабилизатор $V_{i}$ в группе $\widehat{M_{1} \cap O(V)}=\widetilde{M_{0}}$, индуцирует на множестве одномерных подпространств пространства $V_{i}$ группу $P G O\left(V_{i}\right) \cong P G O_{2}^{-}(q) \cong D_{(2, q)(q+1)}$, действующую транзитивно как на множестве прямых $V_{i}$, значения квадратичной формы на векторах которых являются квадратами в поле $F$, так и, в случае нечетного $q$, на множестве прямых $V_{i}$, значения квадратичной формы на ненулевых векторах которых не являются квадратами в поле $F$.

Из равенства $F^{*}\left(M_{0}\right)=O_{p}\left(M_{0}\right)$ с учетом [8, предложение 2.3.6] и [11] следует, что при $\varepsilon_{1}=+$ либо $q=9$ (при этом $p=2$ ), либо $q-$ простое нечетное число $>3$ и $q-1-$ степень 2 (при этом $p=2$ ), либо $q-$ степень 2 и $q-1-$ простое нечетное число (при этом $p=q-1$ ), а при $\varepsilon_{1}=-$ либо $q=8$ (при этом $p=3$ ), либо $q$ - простое нечетное число и $q+1-$ степень 2 (при этом $p=2$ ), либо $q$ - степень 2 и $q+1$ - простое нечетное число (при этом $p=q+1$ ).

Согласно (vii), (viii), если $\varepsilon_{1}=+$ и $q \neq 5$ или $\varepsilon_{1}=-$ и $q \neq 3$, то $M_{0}$ - максимальная подгруппа группы $L$, а если $\varepsilon_{1}=+$ и $q=5$ или $\varepsilon_{1}=-$ и $q=3$, то либо $G / L-$ циклическая группа порядка 4 и $G$ сопряжена в $A u t(L)$ с подгруппой группы $P C G O(V)$, либо Inndiag $(L) \leq$ $G$ и $M_{1} \cap \operatorname{Inndiag}(L)$ - максимальная подгруппа группы $\operatorname{Inndiag}(L)$.

Положим $C_{1}=\left\{h \in M_{0} \mid h\left(V_{i}\right)=V_{i}\right.$ для всех $\left.1 \leq i \leq 4\right\}$ - ядро действия $M_{0}$ на множестве $\left\{V_{1}, V_{2}, V_{3}, V_{4}\right\}, C_{2}=g C_{1} g^{-1}$ - ядро действия $M_{2} \cap L$ на множестве $\left\{g\left(V_{1}\right), g\left(V_{2}\right), g\left(V_{3}\right), g\left(V_{4}\right)\right\}$.

Так как $\left\{V_{1}, V_{2}, V_{3}, V_{4}\right\} \neq\left\{g\left(V_{1}\right), g\left(V_{2}\right), g\left(V_{3}\right), g\left(V_{4}\right)\right\}$ (поскольку $M_{1} \neq M_{2}$ ), будем, не теряя общности, предполагать, что $g\left(V_{1}\right) \notin\left\{V_{1}, V_{2}, V_{3}, V_{4}\right\}$. Тогда найдется $w \in \tilde{g}\left(V_{1}\right)$ такой, что $w=w_{1}+w_{2}+w_{3}+w_{4}$, где $w_{i} \in V_{i}$ для каждого $1 \leq i \leq 4$, причем $w_{k} \neq 0 \neq w_{l}$ для некоторых $1 \leq k<l \leq 4$.

Рассмотрим отдельно случаи четного и нечетного $q$.

Предположим, что q четно. Если $G=L$, то сохраняют силу рассуждения, используемые для рассмотрения случая $m=2$ при четном $q$ из доказательства леммы 2.4 (для получения аналога включения (2.1) достаточно заметить, что $p$ нечетно и, следовательно, $\left.O_{p}\left(S_{4}\right)=1\right)$.

В общем случае (без предположения $G=L)$ с учетом лемм 3.1 и 1.1 для $\left(G, M_{1}, M_{2}\right) \in \Pi$ имеем $\left(L, M_{1} \cap L, M_{2} \cap L\right) \in \Pi$, но последнее противоречит уже рассмотренному случаю $G=L$, $q$ четно.

Предположим, что q нечетно. В этом случае $p=2$ (см. выше).

Для $i \in\{1,2\}$ положим $N_{i}=\Omega\left(Z\left(O_{2}\left(M_{i} \cap L\right)\right)\right.$ ) (таким образом, $\left.N_{2}=g N_{1} g^{-1}\right)$. Из строения группы $M_{i} \cap L, i \in\{1,2\}$, следует, что $N_{i}$ - элементарная группа порядка 4 , неединичные элементы которой транзитивно переставляются подгруппой порядка 3 группы $M_{i} \cap L$. Отсюда вытекает, что $N_{i}$ содержится в каждой неединичной нормальной подгруппе группы $M_{i} \cap L$, $i \in\{1,2\}$. В частности, для $i \in\{1,2\}$ с учетом $1 \neq K_{i} \cap L \unlhd M_{i} \cap L$ (см. лемму 3.1) имеем

$$
N_{i} \leq K_{i} \cap L
$$

что влечет

$$
N_{1} \leq O_{2}\left(\left(M_{2}, M_{1}\right)^{1} \cap L\right) \text { и } N_{2} \leq O_{2}\left(\left(M_{1}, M_{2}\right)^{1} \cap L\right) .
$$

Далее, с учетом $[P G O(V), P G O(V)]=O(V)$ для произвольных различных $1 \leq i_{1}, i_{2} \leq 4$ в группе $\Omega(V)$ содержится элемент $h_{i_{1}, i_{2}}$, который действует тождественно на каждом из подпространств $V_{i}, 1 \leq i \leq 4, i_{1} \neq i \neq i_{2}$, векторного пространства $V$ и инвертирует подпространства $V_{i_{1}}$ и $V_{i_{2}}$. Ясно, что $N_{1}=\left\{1, \bar{h}_{1,2}, \bar{h}_{1,3}, \bar{h}_{1,4}\right\}$ (если $\left\{i_{1}, i_{2}, i_{1}^{\prime}, i_{2}^{\prime}\right\}=\{1,2,3,4\}$, то $\bar{h}_{i_{1}^{\prime}, i_{2}^{\prime}}=\bar{h}_{i_{1}, i_{2}}$ ).

Докажем, что

$$
\left\langle N_{1}, N_{2}\right\rangle \leq C_{1} \cap C_{2}
$$


Покажем, что $N_{1} \leq C_{2}$. Предположим противное, и пусть $a \in N_{1} \backslash C_{2}$. Поскольку $N_{1} \leq O_{2}\left(\left(M_{2}, M_{1}\right)^{1} \cap L\right)$, тогда нормальная 2-подгруппа $O_{2}\left(\left(M_{2}, M_{1}\right)^{1} \cap L\right)$ группы $M_{2}$ индуцирует на $\left\{\tilde{g}\left(V_{1}\right), \tilde{g}\left(V_{2}\right), \tilde{g}\left(V_{3}\right), \tilde{g}\left(V_{4}\right)\right\}$ регулярную элементарную абелеву группу порядка 4 . Таким образом, не теряя общности, можно предполагать, что $\tilde{a}\left(\tilde{g}\left(V_{1}\right)\right)=\tilde{g}\left(V_{2}\right), \tilde{a}\left(\tilde{g}\left(V_{3}\right)\right)=\tilde{g}\left(V_{4}\right)$ и, кроме того, имеется $b \in O_{2}\left(\left(M_{2}, M_{1}\right)^{1} \cap L\right)$, для которого $\tilde{b}\left(\tilde{g}\left(V_{1}\right)\right)=\tilde{g}\left(V_{3}\right)$ и $\tilde{b}\left(\tilde{g}\left(V_{2}\right)\right)=\tilde{g}\left(V_{4}\right)$. В группе $S O(V)$ содержится элемент $x$, действующий тождественно на каждом из подпространств $\tilde{g}\left(V_{2}\right), \tilde{g}\left(V_{3}\right), \tilde{g}\left(V_{4}\right)$ и стабилизирующий подпространство $\tilde{g}\left(V_{1}\right)$, на котором он индуцирует порождающий циклической группы $S O\left(\tilde{g}\left(V_{1}\right)\right)$ (порядка $q-\varepsilon_{1} 1$ ). Заметим, что $x \in \widetilde{M}_{2}$ при $\varepsilon_{1}=+, q=5$ и при $\varepsilon_{1}=-, q=3$, так как в этих случаях $S O(V) \leq \tilde{G}$. В общем же случае (при $\varepsilon_{1}=+, q \geq 5$ и при $\varepsilon_{1}=-, q \geq 3$ ) имеем $x^{2} \in \widehat{M_{2} \cap L}$. Положим $y=x^{2}$ при $\varepsilon_{1}=+, q>5$ и при $\varepsilon_{1}=-, q>3$ (т. е. при $\varepsilon_{1}=+, q \geq 9$ и при $\varepsilon_{1}=-, q \geq 7$ ) и положим $y=x$ при $\varepsilon_{1}=+, q=5$ и при $\varepsilon_{1}=-, q=3$. Тогда элемент $[[y, \tilde{b}], \tilde{a}]$ содержится в $\widetilde{N}_{1}$ (так как $[y, \tilde{b}] \in\left[\widetilde{M_{2}}, \widehat{O_{2}\left(\left(M_{2}, M_{1}\right)^{1} \cap L\right)}\right] \leq \widehat{O_{2}\left(\left(M_{2}, M_{1}\right)^{1} \cap L\right)} \leq \widetilde{M}_{0}$ и $\left.\left[\widetilde{M}_{0}, \tilde{a}\right] \leq\left[\widetilde{M_{0}}, \widetilde{N_{1}}\right] \leq \widetilde{N_{1}}\right)$, причем (в силу выбора $a$ и $b$ ) для каждого $1 \leq i \leq 4$ он стабилизирует $\tilde{g}\left(V_{i}\right)$ и индуцирует на нем квадрат порождающего циклической группы $S O\left(\tilde{g}\left(V_{i}\right)\right)$ при $\varepsilon_{1}=+, q>5$ и при $\varepsilon_{1}=-, q>3$ и порождающий циклической группы $S O\left(\tilde{g}\left(V_{i}\right)\right)$ при $\varepsilon_{1}=+, q=5$ и при $\varepsilon_{1}=-, q=3$. Это противоречит тому, что $\widetilde{N_{1}}=Z(\tilde{G}) \cup h_{1,2} Z(\tilde{G}) \cup h_{1,3} Z(\tilde{G}) \cup h_{1,4} Z(\tilde{G})$.

Включение $N_{2} \leq C_{1}$ доказывается аналогично.

Имея в виду использование леммы 1.1 для анализа общего случая (см. конец рассмотрения случаев (vii) и (viii)), мы будем сначала предполагать выполненным следующее условие (переход к рассмотрению общего случая будет явно оговорен):

$\left(^{*}\right)$ если $\varepsilon_{1}=+u q>5$ или $\varepsilon_{1}=-u q>3$, то $G=L$; если $\varepsilon_{1}=+u q=5$ или $\varepsilon_{1}=-u q=3$, то $G \leq P C G O(V)$, причем либо $G / L-$ чиклическая группа порлдка 4 , либо $G=\operatorname{Inndiag}(L)$.

Начальный этап рассмотрения случая $\left(^{*}\right)$, по существу, дословно совпадает с одним из этапов рассмотрения случая $m=2$ при доказательстве леммы 2.4 , но ряд используемых символов имеет другое значение.

Покажем, что если $\tilde{g}\left(V_{i}\right) \notin\left\{V_{1}, V_{2}, V_{3}, V_{4}\right\}$, где $1 \leq i \leq 4$, то для некоторых $1 \leq i^{\prime}<i^{\prime \prime} \leq 4$ имеем $\operatorname{dim}\left(\tilde{g}\left(V_{i}\right) \cap V_{i^{\prime}}\right)=1=\operatorname{dim}\left(\tilde{g}\left(V_{i}\right) \cap V_{i^{\prime \prime}}\right)$, и что, аналогично, если $V_{i} \notin\left\{\tilde{g}\left(V_{1}\right), \tilde{g}\left(V_{2}\right), \tilde{g}\left(V_{3}\right), \tilde{g}\left(V_{4}\right)\right\}$, где $1 \leq i \leq 4$, то для некоторых $1 \leq i^{\prime}<i^{\prime \prime} \leq 4$ имеем $\operatorname{dim}\left(V_{i} \cap \tilde{g}\left(V_{i^{\prime}}\right)\right)=1=\operatorname{dim}\left(V_{i} \cap \tilde{g}\left(V_{i^{\prime \prime}}\right)\right)$.

Пусть $\tilde{g}\left(V_{i}\right) \notin\left\{V_{1}, V_{2}, V_{3}, V_{4}\right\}$, где $1 \leq i \leq 4$. Тогда найдется $v \in \tilde{g}\left(V_{i}\right)$ такой, что $v=$ $v_{1}+v_{2}+v_{3}+v_{4}$, где $v_{j} \in V_{j}$ для всех $1 \leq j \leq 4$, причем $v_{i^{\prime}} \neq 0 \neq v_{i^{\prime \prime}}$ для некоторых $1 \leq i^{\prime}<i^{\prime \prime} \leq 4$. Докажем, что $\tilde{g}\left(V_{i}\right) \cap V_{i^{\prime}}=\left\langle v_{i^{\prime}}\right\rangle$ и $\tilde{g}\left(V_{i}\right) \cap V_{i^{\prime \prime}}=\left\langle v_{i^{\prime \prime}}\right\rangle$, для чего, очевидно, достаточно доказать, что $\tilde{g}\left(V_{i}\right)$ содержит $v_{i^{\prime}}$ и $v_{i^{\prime \prime}}$. Но с учетом $h_{i^{\prime}, i^{\prime \prime}} \in \widetilde{C_{2}}$ (см. (3.1)) имеем $v_{i^{\prime}}+v_{i^{\prime \prime}}=\frac{1}{2}\left(v-h_{i^{\prime}, i^{\prime \prime}}(v)\right) \in \tilde{g}\left(V_{i}\right)$, что с учетом $h_{i^{\prime}, j^{\prime}} \in \widetilde{C_{2}}$ для $1 \leq j^{\prime} \leq 4, i_{1}^{\prime} \neq j^{\prime} \neq i^{\prime \prime}$ (см. 3.1) влечет $v_{i^{\prime}}=\frac{1}{2}\left(v_{i^{\prime}}+v_{i^{\prime \prime}}-h_{i^{\prime}, j^{\prime}}\left(v_{i^{\prime}}+v_{i^{\prime \prime}}\right)\right) \in \tilde{g}\left(V_{i}\right)$ и $v_{i^{\prime \prime}}=\frac{1}{2}\left(v_{i^{\prime}}+v_{i^{\prime \prime}}+h_{i^{\prime}, j^{\prime}}\left(v_{i^{\prime}}+v_{i^{\prime \prime}}\right)\right) \in \tilde{g}\left(V_{i}\right)$, завершая доказательство первой части утверждения. Вторая часть утверждения доказывается сходным образом. Пусть $V_{i} \notin\left\{\tilde{g}\left(V_{1}\right), \tilde{g}\left(V_{2}\right), \tilde{g}\left(V_{3}\right), \tilde{g}\left(V_{4}\right)\right\}$, где $1 \leq i \leq 4$. Тогда найдется $v \in V_{i}$ такой, что $v=v_{1}+v_{2}+v_{3}+v_{4}$, где $v_{j} \in \tilde{g}\left(V_{j}\right)$ для всех $1 \leq j \leq 4$, причем $v_{i^{\prime}} \neq 0 \neq v_{i^{\prime \prime}}$ для некоторых $1 \leq i^{\prime}<i^{\prime \prime} \leq 4$. Докажем, что $V_{i} \cap \tilde{g}\left(V_{i^{\prime}}\right)=\left\langle v_{i^{\prime}}\right\rangle$ и $V_{i} \cap \tilde{g}\left(V_{i^{\prime \prime}}\right)=\left\langle v_{i^{\prime \prime}}\right\rangle$, для чего, очевидно, достаточно доказать, что $V_{i}$ содержит $v_{i^{\prime}}$ и $v_{i^{\prime \prime}}$. Но с учетом $\tilde{g} h_{i^{\prime}, i^{\prime \prime}} \tilde{g}^{-1} \in \widetilde{N_{2}} \leq$ $\widetilde{C_{1}}$ (см. (3.1)) имеем $v_{i^{\prime}}+v_{i^{\prime \prime}}=\frac{1}{2}\left(v-\tilde{g} h_{i^{\prime}, i^{\prime \prime}} \tilde{g}^{-1}(v)\right) \in V_{i}$, что с учетом $\tilde{g} h_{i^{\prime}, j^{\prime}} \tilde{g}^{-1} \in \widetilde{C_{1}}$ для $1 \leq j^{\prime} \leq 4, i_{1}^{\prime} \neq j^{\prime} \neq i^{\prime \prime}$ (см. (3.1)) влечет $v_{i^{\prime}}=\frac{1}{2}\left(v_{i^{\prime}}+v_{i^{\prime \prime}}-\tilde{g} h_{i^{\prime}, j^{\prime}} \tilde{g}^{-1}\left(v_{i^{\prime}}+v_{i^{\prime \prime}}\right)\right) \in V_{i}$ и $v_{i^{\prime \prime}}=\frac{1}{2}\left(v_{i^{\prime}}+v_{i^{\prime \prime}}+\tilde{g} h_{i^{\prime}, j^{\prime}} \tilde{g}^{-1}\left(v_{i^{\prime}}+v_{i^{\prime \prime}}\right)\right) \in V_{i}$, завершая доказательство утверждения.

Из доказанного утверждения вытекает справедливость следующих утверждений: $w=$ $w_{k}+w_{l}, \tilde{g}\left(V_{1}\right)=\left\langle w_{k}, w_{l}\right\rangle,\left\langle w_{k}\right\rangle=V_{k} \cap \tilde{g}\left(V_{1}\right)$ и $\left\langle w_{l}\right\rangle=V_{l} \cap \tilde{g}\left(V_{1}\right)$, причем $w_{k}$ и $w_{l}$ не являются сингулярными (поскольку $\left(w_{k}, w_{l}\right)=0$, а $\tilde{g}\left(V_{1}\right)$ невырождено) и лишь $\left\langle w_{k}\right\rangle$ и $\left\langle w_{l}\right\rangle$ являются одномерными подпространствами как некоторых $V_{i}, 1 \leq i \leq 4$, так и $\tilde{g}\left(V_{1}\right)$. Кроме того, 
поскольку $\operatorname{dim}\left(V_{k} \cap g\left(V_{1}\right)\right)=1$, из доказанного утверждения вытекает существование такого $2 \leq i_{0} \leq 4$, что $\operatorname{dim}\left(V_{k} \cap g\left(V_{i_{0}}\right)\right)=1$.

Пусть $1 \leq j \leq 4, k \neq j \neq l$. Тогда элемент $h_{k, j}$ инвертирует вектор $w_{k}$ и стабилизирует вектор $w_{l}$. Ясно, что $h_{k, j}$ нетривиально действует на множестве одномерных подпространств из $\tilde{g}\left(V_{1}\right)=\left\langle w_{k}, w_{l}\right\rangle$ и, следовательно, не стабилизирует отличных от $\left\langle w_{k}\right\rangle,\left\langle w_{l}\right\rangle$ одномерных подпространств из $\tilde{g}\left(V_{1}\right)$.

Если $\varepsilon_{1}=+$ и $q>5$ (и тогда, см. выше, $q \geq 9$ ) или $\varepsilon_{1}=-$ и $q>3$ (и тогда, см. выше, $q \geq 7)$, то для группы $R_{1, j^{\prime}}^{g}$, где $j^{\prime} \in\{2,3,4\} \backslash\left\{i_{0}\right\}$, стабилизирующей все одномерные подпространства пространств $\tilde{g}\left(V_{i}\right)$, где $i \in\{2,3,4\} \backslash\left\{j^{\prime}\right\}$, содержащая $\left\langle w_{k}\right\rangle$ орбита на множестве одномерных подпространств пространства $\tilde{g}\left(V_{1}\right)$ имеет длину $>2$, и потому для некоторого $h \in R_{1, j^{\prime}}^{g}$ элемент $\bar{h}_{k, j}$ не стабилизирует $h\left(\left\langle w_{k}\right\rangle\right)$, т. е.

$$
h^{-1} \bar{h}_{k, j} h\left(\left\langle w_{k}\right\rangle\right) \neq\left\langle w_{k}\right\rangle \text {. }
$$

В силу $h \in R_{1, j^{\prime}}^{g} \leq M_{1}^{g}=M_{2}$ и $\bar{h}_{k, j} \in N_{1} \leq\left(M_{2}, M_{1}\right)^{1}$ имеем $h^{-1} \bar{h}_{k, j} h \leq\left(M_{2}, M_{1}\right)^{1} \leq M_{1}$. Таким образом, элемент $\tilde{h}^{-1} h_{k, j} \tilde{h}$ стабилизирует разложение $V=V_{1}+V_{2}+V_{3}+V_{4}$. Кроме того, элемент $\tilde{h}^{-1} h_{k, j} \tilde{h}$ стабилизирует подпространство $\tilde{g}\left(V_{1}\right)$ пространства $V$, поскольку элемент $\tilde{h}$ и (в силу (3.1)) элемент $h_{k, j}$ стабилизируют $\tilde{g}\left(V_{1}\right)$. Так как лишь $\left\langle w_{k}\right\rangle$ и $\left\langle w_{l}\right\rangle$ являются одномерными подпространствами как некоторых $V_{i}, 1 \leq i \leq 4$, так и $\tilde{g}\left(V_{1}\right)$, из (3.2) следует, что $\tilde{h}^{-1} h_{k, j} \tilde{h}\left(\left\langle w_{k}\right\rangle\right)=\left\langle w_{l}\right\rangle$, а значит, $\tilde{h}^{-1} h_{k, j} \tilde{h}\left(V_{k}\right)=V_{l}$. Однако последнее равенство противоречит равенству $\tilde{h}^{-1} h_{k, j} \tilde{h}\left(V_{k} \cap \tilde{g}\left(V_{i_{0}}\right)\right)=V_{k} \cap \tilde{g}\left(V_{i_{0}}\right)$, которое справедливо в силу того, что элемент $\tilde{h}$ стабилизирует каждое одномерное подпространство пространства $\tilde{g}\left(V_{i_{0}}\right)$ и элемент $h_{k, j}$ стабилизирует каждое одномерное подпространство пространства $V_{k}$.

Итак, (при условии $\left.\left(^{*}\right)\right)$ либо $\varepsilon_{1}=+$ и $q=5$, либо $\varepsilon_{1}=-$ и $q=3$.

Пусть $\varepsilon_{1}=+$ и $q=5$. Тогда для $1 \leq i \leq 4$ группа $P G O\left(V_{i}\right)$ имеет на множестве несингулярных прямых пространства $V_{i}$ две орбиты длины 2. А именно, если $\{e, f\}-$ гиперболическая пара гиперболической плоскости $V_{i}$, то $P G O\left(V_{i}\right)$-орбитами на множестве несингулярных прямых $V_{i}$ являются $X_{i}^{-}=\{\langle e+f\rangle,\langle e-f\rangle\}$ и $X_{i}^{+}=\{\langle e+2 f\rangle,\langle e-2 f\rangle\}$, т.е. в точности все пары ортогональных несингулярных прямых. Если $v$ - ненулевой вектор прямой из $X_{i}^{-}$, то $(v, v)$ не есть квадрат в поле $F$, а если $v$ - вектор прямой из $X_{i}^{+}$, то $(v, v)$ есть квадрат в поле $F$.

Сходная ситуация имеет место при $\varepsilon_{1}=-$ и $q=3$. В этом случае для $1 \leq i \leq 4$ группа $P G O\left(V_{i}\right)$ имеет на множестве прямых пространства $V_{i}$ две орбиты $X_{i}^{-}$и $X_{i}^{+}$длины 2 со следующими свойствами: если $v-$ ненулевой вектор прямой из $X_{i}^{-}$, то $(v, v)$ не есть квадрат в поле $F$, а если $v$ - вектор прямой из $X_{i}^{+}$, то $(v, v)$ есть квадрат в поле $F$. При этом множества $X_{i}^{-}, X_{i}^{+}$и только они являются парами ортогональных прямых $V_{i}$.

Дальнейшие рассуждения применимы как в случае $\varepsilon_{1}=+$ и $q=5$, так и в случае $\varepsilon_{1}=-$ и $q=3$. Для $1 \leq i \leq 4$ ограничение на множество прямых $V_{i}$ стабилизатора этого множества в группе $M_{1}$ содержит группу $P G O\left(V_{i}\right)$ (см. выше), но отлично от $P G O\left(V_{i}\right)$, что можно усмотреть из [15, предложение 6.2.11] и [8] (см. аналогичное место в доказательстве леммы 2.4 , случай $m=2$ ). Следовательно, для $1 \leq i \leq 4$ ограничение на множество прямых $V_{i}$ стабилизатора этого множества в группе $M_{1}$ есть группа $P C G O\left(V_{i}\right)$. Но тогда, учитывая, что $\widetilde{M}_{0}$ индуцирует на множестве слагаемых разложения $V=V_{1}+V_{2}+V_{3}+V_{4}$ симметрическую группу $S_{4}$, заключаем, что в $M_{1}$ имеется элемент $h \in P C G O(V) \backslash P G O(V)$, который для каждого $1 \leq i \leq 4$ оставляет на месте множество прямых подпространства $V_{i}$, меняя при этом местами множества $X_{i}^{-}$и $X_{i}^{+}$.

Пусть $\tilde{g}\left(V_{i}\right) \notin\left\{V_{1}, V_{2}, V_{3}, V_{4}\right\}$, где $1 \leq i \leq 4$. Тогда, как было доказано выше, найдутся $1 \leq i^{\prime}, i^{\prime \prime} \leq 4, i^{\prime} \neq i^{\prime \prime}$, такие, что $\operatorname{dim}\left(\tilde{g}\left(V_{i}\right) \cap V_{i^{\prime}}\right)=1=\operatorname{dim}\left(\tilde{g}\left(V_{i}\right) \cap V_{i^{\prime \prime}}\right)$. Покажем, что для некоторого $1 \leq i^{*} \leq 4, i^{*} \neq i$, также имеем $\operatorname{dim}\left(\tilde{g}\left(V_{i^{*}}\right) \cap V_{i^{\prime}}\right)=1=\operatorname{dim}\left(\tilde{g}\left(V_{i^{*}}\right) \cap V_{i^{\prime \prime}}\right)$, причем $\left\{\tilde{g}\left(V_{i}\right) \cap V_{i^{\prime}}, \tilde{g}\left(V_{i^{*}}\right) \cap V_{i^{\prime}}\right\}=X_{i^{\prime}}^{\xi}$ и $\left\{\tilde{g}\left(V_{i}\right) \cap V_{i^{\prime \prime}}, \tilde{g}\left(V_{i^{*}}\right) \cap V_{i^{\prime \prime}}\right\}=X_{i^{\prime \prime}}^{\xi}$ для некоторого $\xi \in\{-,+\}$.

Так как $V_{i^{\prime}} \notin\left\{\tilde{g}\left(V_{1}\right), \tilde{g}\left(V_{2}\right), \tilde{g}\left(V_{3}\right), \tilde{g}\left(V_{4}\right)\right\}$ (поскольку $\operatorname{dim}\left(\tilde{g}\left(V_{i}\right) \cap V_{i^{\prime}}\right)=1$ ), то из доказанного выше следует существование такого $1 \leq i^{*} \leq 4, i^{*} \neq i$, что $\operatorname{dim}\left(\tilde{g}\left(V_{i^{*}}\right) \cap V_{i^{\prime}}\right)=1$. Ясно, что 
$\tilde{g}\left(V_{i}\right) \cap V_{i^{\prime}}$ и $\tilde{g}\left(V_{i^{*}}\right) \cap V_{i^{\prime}}$ несингулярны (поскольку $V_{i^{\prime}}=\left\langle\tilde{g}\left(V_{i}\right) \cap V_{i^{\prime}}, \tilde{g}\left(V_{i^{*}}\right) \cap V_{i^{\prime}}\right\rangle$ невырождено). Пусть $j^{\prime} \in\{1, \ldots, 4\} \backslash\left\{i, i^{*}\right\}$. Тогда элемент $\tilde{g} h_{i, j^{\prime}} \tilde{g}^{-1}$ из $\widetilde{N_{2}} \leq \widetilde{C_{1}} \cap \widehat{O_{2}\left(\left(M_{1}, M_{2}\right)^{1}\right)}$ инвертирует $\tilde{g}\left(V_{i}\right)$ и централизует $\tilde{g}\left(V_{i^{*}}\right)$, а следовательно, меняет местами несингулярные прямые подпространства $V_{i^{\prime}}$ пространства $V$, отличные от $\tilde{g}\left(V_{i}\right) \cap V_{i^{\prime}}$ и $\tilde{g}\left(V_{i^{*}}\right) \cap V_{i^{\prime}}$. Поскольку определенный выше элемент $h$ группы $M_{1}$ меняет местами множества $X_{i}^{-}$и $X_{i}^{+}$, отсюда с учетом (3.1) следует, что содержащийся в $\widetilde{C_{1}} \cap \widehat{O_{2}\left(\left(M_{1}, M_{2}\right)^{1}\right)} \leq \widetilde{C_{1}} \cap \widetilde{M_{2}}$ элемент $\tilde{h} \tilde{g} h_{i, j^{\prime}} \tilde{g}^{-1} \tilde{h}^{-1}$ меняет местами прямые $\tilde{g}\left(V_{i}\right) \cap V_{i^{\prime}}$ и $\tilde{g}\left(V_{i^{*}}\right) \cap V_{i^{\prime}}$. Но тогда элемент $\tilde{h} \tilde{g} h_{i, j^{\prime}} \tilde{g}^{-1} \tilde{h}^{-1}$ меняет местами $\tilde{g}\left(V_{i}\right)$ и $\tilde{g}\left(V_{i^{*}}\right)$, оставляя на месте $V_{i^{\prime}}$ и $V_{i^{\prime \prime}}$. Отсюда следует, что $\operatorname{dim}\left(\tilde{g}\left(V_{i^{*}}\right) \cap V_{i^{\prime \prime}}\right)=\operatorname{dim}\left(\tilde{g}\left(V_{i^{*}}\right) \cap V_{i^{\prime}}\right)=1$. Кроме того, с учетом $\tilde{h} \tilde{g} h_{i, j^{\prime}} \tilde{g}^{-1} \tilde{h}^{-1} \in \tilde{L}$ отсюда следует, что $\left\{\tilde{g}\left(V_{i}\right) \cap V_{i^{\prime}}, \tilde{g}\left(V_{i^{*}}\right) \cap V_{i^{\prime}}\right\}=X_{i^{\prime}}^{\xi}$ и $\left\{\tilde{g}\left(V_{i}\right) \cap V_{i^{\prime \prime}}, \tilde{g}\left(V_{i^{*}}\right) \cap V_{i^{\prime \prime}}\right\}=X_{i^{\prime \prime}}^{\xi^{\prime}}$ для некоторых $\xi, \xi^{\prime} \in\{-,+\}$. Остается доказать, что $\xi=\xi^{\prime}$. Элемент $h_{i^{\prime}, j^{\prime}} \in \tilde{L}$ инвертирует $V_{i^{\prime}}$ и централизует $V_{i^{\prime \prime}}$, а следовательно меняет местами несингулярные прямые подпространства $\tilde{g}\left(V_{i}\right)$ пространства $V$, отличные от $\tilde{g}\left(V_{i}\right) \cap V_{i^{\prime}}$ и $\tilde{g}\left(V_{i}\right) \cap V_{i^{\prime \prime}}$. Но тогда $\tilde{g}\left(V_{i}\right) \cap V_{i^{\prime}} \in X_{i^{\prime}}^{\xi^{\prime \prime}}$ и $\tilde{g}\left(V_{i}\right) \cap V_{i^{\prime \prime}} \in X_{i^{\prime \prime}}^{\xi^{\prime \prime}}$ для некоторого $\xi^{\prime \prime} \in\{-,+\}$. Таким образом, $\xi=\xi^{\prime \prime}=\xi^{\prime}$.

Так как $\operatorname{dim}\left(\tilde{g}\left(V_{1}\right) \cap V_{k}\right)=1=\operatorname{dim}\left(\tilde{g}\left(V_{1}\right) \cap V_{l}\right)$ (см. выше), то из доказанного утверждения следует существование такого $2 \leq i_{0} \leq 4$, что $\operatorname{dim}\left(\tilde{g}\left(V_{i_{0}}\right) \cap V_{k}\right)=1=\operatorname{dim}\left(\tilde{g}\left(V_{i_{0}}\right) \cap V_{l}\right)$, причем $\left\{\tilde{g}\left(V_{1}\right) \cap V_{k}, \tilde{g}\left(V_{i_{0}}\right) \cap V_{k}\right\}=X_{k}^{\xi_{0}}$ и $\left\{\tilde{g}\left(V_{1}\right) \cap V_{l}, \tilde{g}\left(V_{i_{0}}\right) \cap V_{l}\right\}=X_{l}^{\xi_{0}}$ для некоторого $\xi_{0} \in\{-,+\}$.

Если $a \in C_{1} \cap O_{2}\left(\left(M_{1}, M_{2}\right)^{1}\right)$, то элемент $а$ стабилизирует каждое из множеств прямых $X_{i}^{-}$ и $X_{i}^{+}$для каждого $1 \leq i \leq 4$. Покажем, что при этом для любого $\xi \in\{-,+\}$ элемент $а$ либо стабилизирует каждую прямую из $\cup_{1 \leq i \leq 4} X_{i}^{\xi}$, либо сдвигает каждую прямую из $\cup_{1 \leq i \leq 4} X_{i}^{\xi}$. Предположим, что, напротив, для некоторого $a \in C_{1} \cap O_{2}\left(\left(M_{1}, M_{2}\right)^{1}\right)$ и некоторого $\xi \in\{-,+\}$ найдутся $1 \leq i^{\prime}, i^{\prime \prime} \leq 4, i^{\prime} \neq i^{\prime \prime}$, такие, что элемент а меняет местами прямые из $X_{i^{\prime}}^{\xi}$ и стабилизирует каждую прямую из $X_{i^{\prime \prime}}^{\xi}$. Так как группа $\widetilde{M}_{0}$ индуцирует на $\left\{V_{1}, V_{2}, V_{3}, V_{4}\right\}$ симметрическую группу $S_{4}$, то, заменяя в случае необходимости элемент $a$ на элемент, сопряженный с ним в группе $M_{1}$, будем без потери общности предполагать, что $i^{\prime}=k$ и $i^{\prime \prime}=l$. Далее, заменяя в случае необходимости элемент $a$ на элемент $h a h^{-1}$, будем без потери общности предполагать, кроме того, что $\xi=\xi_{0}$. Но тогда $\tilde{a} \tilde{g}\left(V_{1}\right) \notin\left\{\tilde{g}\left(V_{1}\right), \ldots, \tilde{g}\left(V_{t}\right)\right\}$ и, следовательно, $a \notin M_{2} \cap L$, что противоречит $a \in C_{1} \cap O_{2}\left(\left(M_{1}, M_{2}\right)^{1}\right) \leq M_{2} \cap L$.

Для произвольных $1 \leq i_{1}, i_{2} \leq 4, i_{1} \neq i_{2}$, элемент $g \bar{h}_{i_{1}, i_{2}} g^{-1} \in N_{2} \leq C_{1} \cap O_{2}\left(\left(M_{1}, M_{2}\right)^{1}\right)$ (см. (3.1)) стабилизирует каждое из множеств прямых $X_{i}^{-}$и $X_{i}^{+}$для любого $1 \leq i \leq 4$, причем, учитывая только что доказанное, для произвольного $\xi \in\{-,+\}$ либо стабилизирует каждую прямую из $\cup_{1 \leq i \leq 4} X_{i}^{\xi}$, либо сдвигает каждую прямую из $\cup_{1 \leq i \leq 4} X_{i}^{\xi}$. В частности, для $i \in\{2,3,4\} \backslash\left\{i_{0}\right\}$ и $\xi^{\prime} \in\{-,+\} \backslash\left\{\xi_{0}\right\}$ ( $i_{0}$ и $\xi_{0}$ были определены выше) элемент $\tilde{g} h_{1, i} \tilde{g}^{-1}$, который инвертирует подпространство $\tilde{g}\left(V_{1}\right)$ пространства $V$ и централизует подпространство $\tilde{g}\left(V_{i_{0}}\right)$ пространства $V$, меняет местами прямые из каждого из множеств $X_{1}^{\xi^{\prime}}, X_{2}^{\xi^{\prime}}, X_{3}^{\xi^{\prime}}, X_{4}^{\xi^{\prime}}$. Но тогда $\tilde{g}\left(V_{i}\right) \notin\left\{V_{1}, V_{2}, V_{3}, V_{4}\right\}$ (поскольку элемент $\tilde{g} h_{1, i} \tilde{g}^{-1}$ инвертирует подпространство $\tilde{g}\left(V_{i}\right)$ пространства $\left.V\right)$, и потому, как было доказано ранее, найдутся $1 \leq i^{\prime}, i^{\prime \prime} \leq 4, i^{\prime} \neq i^{\prime \prime}$, и $1 \leq i^{*} \leq 4, i^{*} \neq i$, такие, что

$$
\operatorname{dim}\left(\tilde{g}\left(V_{i}\right) \cap V_{i^{\prime}}\right)=\operatorname{dim}\left(\tilde{g}\left(V_{i^{*}}\right) \cap V_{i^{\prime}}\right)=1=\operatorname{dim}\left(\tilde{g}\left(V_{i}\right) \cap V_{i^{\prime \prime}}\right)=\operatorname{dim}\left(\tilde{g}\left(V_{i^{*}}\right) \cap V_{i^{\prime \prime}}\right) .
$$

При этом ясно, что $\left\{1, i_{0}, i, i^{*}\right\}=\{1,2,3,4\}=\left\{k, l, i^{\prime}, i^{\prime \prime}\right\}$.

Покажем, что $\left\{\tilde{g}\left(V_{i}\right) \cap V_{i^{\prime}}, \tilde{g}\left(V_{i^{*}}\right) \cap V_{i^{\prime}}\right\}=X_{i^{\prime}}^{\xi_{0}}$, откуда, как было показано выше, будет следовать, что $\left\{\tilde{g}\left(V_{i}\right) \cap V_{i^{\prime \prime}}, \tilde{g}\left(V_{i^{*}}\right) \cap V_{i^{\prime \prime}}\right\}=X_{i^{\prime \prime}}^{\xi_{0}}$. Мы знаем, что элемент $\tilde{g} h_{1, i} \tilde{g}^{-1}$ меняет местами прямые из каждого из множеств $X_{1}^{\xi^{\prime}}, X_{2}^{\xi^{\prime}}, X_{3}^{\xi^{\prime}}, X_{4}^{\xi^{\prime}}$. Поэтому в случае $\left\{\tilde{g}\left(V_{i}\right) \cap V_{i^{\prime}}, \tilde{g}\left(V_{i^{*}}\right) \cap V_{i^{\prime}}\right\}=$ $X_{i^{\prime}}^{\xi^{\prime}}$ элемент $\tilde{g} h_{1, i} \tilde{g}^{-1}$ меняет местами прямые $\tilde{g}\left(V_{i}\right) \cap V_{i^{\prime}}$ и $\tilde{g}\left(V_{i^{*}}\right) \cap V_{i^{\prime}}$. Но элемент $\tilde{g} h_{1, i} \tilde{g}^{-1}$ инвертирует $\tilde{g}\left(V_{i}\right)$, что дает требуемое противоречие.

Для каждого $1 \leq s \leq 4$ пусть $e_{s}, f_{s}$ - базис подпространства $V_{s}$ пространства $V$ такой, что при $\varepsilon_{1}=+$ и $q=5$ имеют место равенства $\left(e_{s}, e_{s}\right)=0=\left(f_{s}, f_{s}\right)$ и $\left(e_{s}, f_{s}\right)=1$, а при $\varepsilon_{1}=-$ 
и $q=3$ имеют место равенства $\left(e_{s}, e_{s}\right)=1=\left(f_{s}, f_{s}\right)$ и $\left(e_{s}, f_{s}\right)=0$. При $\varepsilon_{1}=+$ и $q=5$ для каждого $1 \leq s \leq 4$ положим $v_{s, 1}=e_{s}+f_{s}, v_{s, 2}=e_{s}-f_{s}, v_{s, 3}=e_{s}+2 f_{s}, v_{s, 4}=e_{s}-2 f_{s}$, а при $\varepsilon_{1}=-$ и $q=3$ для каждого $1 \leq s \leq 4$ положим $v_{s, 1}=e_{s}+f_{s}, v_{s, 2}=e_{s}-f_{s}, v_{s, 3}=e_{s}, v_{s, 4}=f_{s}$.

Пусть $\mathcal{V}:=\left\{\left\{U_{1}, U_{2}, U_{3}, U_{4}\right\} \mid U_{1}, U_{2}, U_{3}, U_{4}\right.$ - невырожденные 2-мерные подпространства пространства $V$ типа $\varepsilon_{1}$ такие, что $\left.V=U_{1} \oplus U_{2} \oplus U_{3} \oplus U_{4}\right\}$.

Положим (как при $\varepsilon_{1}=+$ и $q=5$, так и при $\varepsilon_{1}=-$ и $q=3$ )

$$
\begin{gathered}
\mathcal{V}_{\left\{V_{1}, V_{2}, V_{3}, V_{4}\right\}}^{-}:=\left\{\left\{\left\langle v_{1,1}, v_{s_{1}, 1}\right\rangle,\left\langle v_{1,2}, v_{s_{1}, 2}\right\rangle,\left\langle v_{s_{2}, 1}, v_{s_{3}, 1}\right\rangle,\left\langle v_{s_{2}, 2}, v_{s_{3}, 2}\right\rangle\right\},\right. \\
\left\{\left\langle v_{1,1}, v_{s_{1}, 1}\right\rangle,\left\langle v_{1,2}, v_{s_{1}, 2}\right\rangle,\left\langle v_{s_{2}, 1}, v_{s_{3}, 2}\right\rangle,\left\langle v_{s_{2}, 2}, v_{s_{3}, 1}\right\rangle\right\}, \\
\left\{\left\langle v_{1,1}, v_{s_{1}, 2}\right\rangle,\left\langle v_{1,2}, v_{s_{1}, 1}\right\rangle,\left\langle v_{s_{2}, 1}, v_{s_{3}, 1}\right\rangle,\left\langle v_{s_{2}, 2}, v_{s_{3}, 2}\right\rangle\right\}, \\
\left.\left\{\left\langle v_{1,1}, v_{s_{1}, 2}\right\rangle,\left\langle v_{1,2}, v_{s_{1}, 1}\right\rangle,\left\langle v_{s_{2}, 1}, v_{s_{3}, 2}\right\rangle,\left\langle v_{s_{2}, 2}, v_{s_{3}, 1}\right\rangle\right\} \mid\left\{s_{1}, s_{2}, s_{3}\right\}=\{2,3,4\}, s_{2}<s_{3}\right\}
\end{gathered}
$$

$\left(\mathcal{V}_{\left\{V_{1}, V_{2}, V_{3}, V_{4}\right\}}^{-}\right.$есть двенадцатиэлементное подмножество множества $\mathcal{V}$, состоящее из таких $\left\{U_{1}, U_{2}, U_{3}, U_{4}\right\}$, что для каждого $1 \leq t \leq 4$ найдутся $1 \leq t_{1}<t_{2} \leq 4$ и $1 \leq t^{\prime} \leq 4, t^{\prime} \neq t$, со следующими свойствами: $\left.U_{t} \cap V_{t_{1}} \in X_{t_{1}}^{-}, U_{t^{\prime}} \cap V_{t_{1}} \in X_{t_{1}}^{-}, U_{t} \cap V_{t_{2}} \in X_{t_{2}}^{-}, U_{t^{\prime}} \cap V_{t_{2}} \in X_{t_{2}}^{-}\right)$,

$$
\begin{gathered}
\mathcal{V}_{\left\{V_{1}, V_{2}, V_{3}, V_{4}\right\}}^{+}:=\left\{\left\{\left\langle v_{1,3}, v_{s_{1}, 3}\right\rangle,\left\langle v_{1,4}, v_{s_{1}, 4}\right\rangle,\left\langle v_{s_{2}, 3}, v_{s_{3}, 3}\right\rangle,\left\langle v_{s_{2}, 4}, v_{s_{3}, 4}\right\rangle\right\},\right. \\
\left\{\left\langle v_{1,3}, v_{s_{1}, 3}\right\rangle,\left\langle v_{1,4}, v_{s_{1}, 4}\right\rangle,\left\langle v_{s_{2}, 3}, v_{s_{3}, 4}\right\rangle,\left\langle v_{s_{2}, 4}, v_{s_{3}, 3}\right\rangle\right\}, \\
\left\{\left\langle v_{1,3}, v_{s_{1}, 4}\right\rangle,\left\langle v_{1,4}, v_{s_{1}, 3}\right\rangle,\left\langle v_{s_{2}, 3}, v_{s_{3}, 3}\right\rangle,\left\langle v_{s_{2}, 4}, v_{s_{3}, 4}\right\rangle\right\}, \\
\left.\left\{\left\langle v_{1,3}, v_{s_{1}, 4}\right\rangle,\left\langle v_{1,4}, v_{s_{1}, 3}\right\rangle,\left\langle v_{s_{2}, 3}, v_{s_{3}, 4}\right\rangle,\left\langle v_{s_{2}, 4}, v_{s_{3}, 3}\right\rangle\right\} \mid\left\{s_{1}, s_{2}, s_{3}\right\}=\{2,3,4\}, s_{2}<s_{3}\right\}
\end{gathered}
$$

$\left(\mathcal{V}_{\left\{V_{1}, V_{2}, V_{3}, V_{4}\right\}}^{-}\right.$есть двенадцатиэлементное подмножество множества $\mathcal{V}$, состоящее из таких $\left\{U_{1}, U_{2}, U_{3}, U_{4}\right\}$, что для каждого $1 \leq t \leq 4$ найдутся $1 \leq t_{1}<t_{2} \leq 4$ и $1 \leq t^{\prime} \leq 4, t^{\prime} \neq t$, со следующими свойствами: $\left.U_{t} \cap V_{t_{1}} \in X_{t_{1}}^{+}, U_{t^{\prime}} \cap V_{t_{1}} \in X_{t_{1}}^{+}, U_{t} \cap V_{t_{2}} \in X_{t_{2}}^{+}, U_{t^{\prime}} \cap V_{t_{2}} \in X_{t_{2}}^{+}\right)$.

Из доказанного ранее следует, что $\left\{\tilde{g}\left(V_{1}\right), \tilde{g}\left(V_{2}\right), \tilde{g}\left(V_{3}\right), \tilde{g}\left(V_{4}\right)\right\} \in \mathcal{V}_{\left\{V_{1}, V_{2}, V_{3}, V_{4}\right\}}^{-} \cup \mathcal{V}_{\left\{V_{1}, V_{2}, V_{3}, V_{4}\right\}}^{+}$.

Так как группа $G O(V)$ действует транзитивно на множестве $\mathcal{V}$, то из $c=1$ в (vii) и (viii) следует транзитивность действия на этом множестве и группы $\tilde{L}$. Далее, несложно видеть, что множества $\mathcal{V}_{\left\{V_{1}, V_{2}, V_{3}, V_{4}\right\}}^{-}$и $\mathcal{V}_{\left\{V_{1}, V_{2}, V_{3}, V_{4}\right\}}^{+}$являются $\widehat{M_{1} \cap P S O(V)}$-орбитами на $\mathcal{V}$ (действительно, группа $\widetilde{M}_{1} \cap S O(V)$ индуцирует на множестве $\left\{V_{1}, V_{2}, V_{3}, V_{4}\right\}$ симметрическую группу и, кроме того, в $\widetilde{M}_{1} \cap S O(V)$ имеется элемент, меняющий местами $\left\langle v_{1,1}\right\rangle$ с $\left\langle v_{1,2}\right\rangle$ и $\left\langle v_{1,3}\right\rangle$ с $\left\langle v_{1,4}\right\rangle$ и действующий тождественно на $\left.V_{2}+V_{3}+V_{4}\right)$.

Ранее было показано, что в $M_{1}$ имеется элемент $h \in P C G O(V) \backslash P G O(V)$, который для каждого $1 \leq i \leq t$ оставляет на месте множество прямых подпространства $V_{i}$, меняя при этом местами множества $X_{i}^{-}$и $X_{i}^{+}$. Ясно, что элемент $\tilde{h}$ меняет местами множества $\mathcal{V}_{\left\{V_{1}, V_{2}, V_{3}, V_{4}\right\}}^{-}$и $\mathcal{V}_{\left\{V_{1}, V_{2}, V_{3}, V_{4}\right\}}^{+}$, откуда в силу $\widetilde{M}_{1}=\left\langle\widehat{M_{1} \cap P S O(V)}, \tilde{h}\right\rangle$ (напомним, что мы предполагаем выполненным условие $\left.\left({ }^{*}\right)\right)$ следует, что множество $\mathcal{V}_{\left\{V_{1}, V_{2}, V_{3}, V_{4}\right\}}^{-} \cup \mathcal{V}_{\left\{V_{1}, V_{2}, V_{3}, V_{4}\right\}}^{+}$является $\widetilde{M}_{1}$-орбитой на $\mathcal{V}$. В частности, все тройки вида $\left(G, M_{1}, M_{2}^{\prime}\right)$, где $\widetilde{M_{2}^{\prime}}-$ стабилизатор в группе $\tilde{G}$ разложения $V=U_{1}+U_{2}+U_{3}+U_{4}$ для $\left\{U_{1}, U_{2}, U_{3}, U_{4}\right\} \in \mathcal{V}_{\left\{V_{1}, V_{2}, V_{3}, V_{4}\right\}}^{-} \cup \mathcal{V}_{\left\{V_{1}, V_{2}, V_{3}, V_{4}\right\}}^{+}$, сопряжены между собой элементами из $M_{1}$ и, следовательно, эквивалентны между собой (причем для каждой такой тройки $\left(G, M_{1}, M_{2}^{\prime}\right)$ в $L$ найдется элемент, сопрягающий $M_{1}$ в $\left.M_{2}^{\prime}\right)$. Но нами показано, что при выполнении для рассматриваемой тройки $\left(G, M_{1}, M_{2}\right)$ условия $\left(^{*}\right)$ группа $\widetilde{M}_{2}$ есть стабилизатор в группе $\tilde{G}$ разложения $V=\tilde{g}\left(V_{1}\right)+\tilde{g}\left(V_{2}\right)+\tilde{g}\left(V_{3}\right)+\tilde{g}\left(V_{4}\right)$, где $\left\{\tilde{g}\left(V_{1}\right), \tilde{g}\left(V_{2}\right), \tilde{g}\left(V_{3}\right), \tilde{g}\left(V_{4}\right)\right\} \in$ $\mathcal{V}_{\left\{V_{1}, V_{2}, V_{3}, V_{4}\right\}}^{-} \cup \mathcal{V}_{\left\{V_{1}, V_{2}, V_{3}, V_{4}\right\}}^{+}$. Следовательно, для завершения рассмотрения случаев (vii) и (viii) при выполнении условия $\left({ }^{*}\right)$ остается проверить справедливости п. (b) теоремы для тройки 
$\left(G, M_{1}, M_{2}^{\prime \prime}\right)$, где $G$ удовлетворяет условию $\left(^{*}\right), \widetilde{M_{1}}-$ стабилизатор в $\tilde{G}$ ортогонального разложения $V=\left\langle e_{1}, f_{1}\right\rangle+\left\langle e_{2}, f_{2}\right\rangle+\left\langle e_{3}, f_{3}\right\rangle+\left\langle e_{4}, f_{4}\right\rangle, \tilde{M}_{2}^{\prime \prime}-$ стабилизатор в $\tilde{G}$ ортогонального разложения $V=\left\langle e_{1}+f_{1}, e_{2}+f_{2}\right\rangle+\left\langle e_{1}-f_{1}, e_{2}-f_{2}\right\rangle+\left\langle e_{3}+f_{3}, e_{4}+f_{4}\right\rangle+\left\langle e_{3}-f_{3}, e_{4}-f_{4}\right\rangle$. Такую проверку несложно осуществить, используя GAP (см. [10]). Этим рассмотрение случаев (vii) и (viii) при выполнении условия $\left(^{*}\right)$ завершено.

Теперь мы можем завершить рассмотрение случаев (vii) и (viii) в общем случае (не предполагая выполненным условие $(*))$.

Если $\varepsilon_{1}=+$ и $q>5$ или $\varepsilon_{1}=-$ и $q>3$, то для $\left(G, M_{1}, M_{2}\right) \in \Pi$ с учетом лемм 3.1 и 1.1 имеем $\left(L, M_{1} \cap L, M_{2} \cap L\right) \in \Pi$, что противоречит уже рассмотренному случаю $G=L, \varepsilon_{1}=+$ и $q>5$ или $\varepsilon_{1}=-$ и $q>3$.

Пусть $\varepsilon_{1}=+$ и $q=5$ или $\varepsilon_{1}=-$ и $q=3$. Если $G / L-$ циклическая группа порядка 4 , то, поскольку все (содержащие $L$ ) такие подгруппы $G$ группы $A u t(L)$ сопряжены в группе $A u t(L)$, мы можем предполагать, что $G \leq P C G O(V)$. Но тогда выполняется условие $\left(^{*}\right)$. В силу доказанного ранее получаем, что теорема справедлива в случае, когда $G / L$ - циклическая группа порядка $4, \varepsilon_{1}=+$ и $q=5$ или $\varepsilon_{1}=-$ и $q=3$.

Пусть, наконец, $\varepsilon_{1}=+$ и $q=5$ или $\varepsilon_{1}=-$ и $q=3$, а $G / L$ не есть циклическая группа порядка 4. Тогда согласно (vii), (viii) имеем Inndiag $(L) \unlhd G$. Далее, согласно леммам 3.1 и 1.1 из $\left(G, M_{1}, M_{2}\right) \in \Pi$ следует $\left(\operatorname{Inndiag}(L), M_{1} \cap \operatorname{Inndiag}(L), M_{2} \cap \operatorname{Inndiag}(L)\right) \in \Pi$, причем

$$
\begin{gathered}
\left(M_{1}, M_{2}\right)^{i} \cap \operatorname{Inndiag}(L) \leq\left(M_{1} \cap \operatorname{Inndiag}(L), M_{2} \cap \operatorname{Inndiag}(L)\right)^{i}, \\
\left(M_{2}, M_{1}\right)^{i} \cap \operatorname{Inndiag}(L) \leq\left(M_{2} \cap \operatorname{Inndiag}(L), M_{1} \cap \operatorname{Inndiag}(L)\right)^{i}
\end{gathered}
$$

для $i \in\{1,2\}$. Но для тройки $\left(\operatorname{Inndiag}(L), M_{1} \cap \operatorname{Inndiag}(L), M_{2} \cap \operatorname{Inndiag}(L)\right)$ из П выполняется условие $\left(^{*}\right)$, и из ранее доказанного следует, что

$$
\begin{aligned}
& \left(M_{1} \cap \operatorname{Inndiag}(L), M_{2} \cap \operatorname{Inndiag}(L)\right)^{i} \operatorname{char} M_{1} \cap \operatorname{Inndiag}(L), \\
& \left(M_{2} \cap \operatorname{Inndiag}(L), M_{1} \cap \operatorname{Inndiag}(L)\right)^{i} \operatorname{char} M_{2} \cap \operatorname{Inndiag}(L)
\end{aligned}
$$

для $i \in\{1,2\}$. Таким образом,

$$
\begin{aligned}
& \left(M_{1} \cap \operatorname{Inndiag}(L), M_{2} \cap \operatorname{Inndiag}(L)\right)^{i} \unlhd M_{1}, \\
& \left(M_{2} \cap \operatorname{Inndiag}(L), M_{1} \cap \operatorname{Inndiag}(L)\right)^{i} \unlhd M_{2}
\end{aligned}
$$

для $i \in\{1,2\}$, что влечет

$$
\begin{gathered}
\left(M_{1} \cap \operatorname{Inndiag}(L), M_{2} \cap \operatorname{Inndiag}(L)\right)^{i} \leq\left(M_{1}, M_{2}\right)^{i} \cap \operatorname{Inndiag}(L), \\
\left(M_{2} \cap \operatorname{Inndiag}(L), M_{1} \cap \operatorname{Inndiag}(L)\right)^{i} \leq\left(M_{2}, M_{1}\right)^{i} \cap \operatorname{Inndiag}(L)
\end{gathered}
$$

для $i \in\{1,2\}$. Итак,

$$
\begin{aligned}
& \left(M_{1}, M_{2}\right)^{i} \cap \operatorname{Inndiag}(L)=\left(M_{1} \cap \operatorname{Inndiag}(L), M_{2} \cap \operatorname{Inndiag}(L)\right)^{i}, \\
& \left(M_{2}, M_{1}\right)^{i} \cap \operatorname{Inndiag}(L)=\left(M_{2} \cap \operatorname{Inndiag}(L), M_{1} \cap \operatorname{Inndiag}(L)\right)^{i}
\end{aligned}
$$

для $i \in\{1,2\}$.

Далее, если $\left(M_{1}, M_{2}\right)^{1} \not L L$, то с учетом $G=L M_{1}$ имеем $L<\left(L\left(M_{1}, M_{2}\right)^{1}\right) \cap$ $\operatorname{Inndiag}(L)$. Но тогда $\left(M_{1}, M_{2}\right)^{1} \cap \operatorname{Inndiag}(L) \not \leq L$, что в силу доказанного равенства $\left(M_{1}, M_{2}\right)^{1} \cap \operatorname{Inndiag}(L)=\left(M_{1} \cap \operatorname{Inndiag}(L), M_{2} \cap \operatorname{Inndiag}(L)\right)^{1}$ противоречит включению $\left(M_{1} \cap \text { Inndiag }(L), M_{2} \cap \text { Inndiag }(L)\right)^{1} \leq L$, справедливость которого следует (с учетом $\left.\left(\operatorname{Inndiag}(L), M_{1} \cap \operatorname{Inndiag}(L), M_{2} \cap \operatorname{Inndiag}(L)\right) \in \Pi\right)$ из ранее рассмотренного случая (*). Таким образом, $\left(M_{1}, M_{2}\right)^{1} \leq L$ и, следовательно,

$$
\left(M_{1}, M_{2}\right)^{i}=\left(M_{1} \cap \operatorname{Inndiag}(L), M_{2} \cap \operatorname{Inndiag}(L)\right)^{i}
$$


для $i \in\{1,2\}$. Аналогично доказывается, что $\left(M_{2}, M_{1}\right)^{1} \leq L$ и

$$
\left(M_{2}, M_{1}\right)^{i}=\left(M_{2} \cap \operatorname{Inndiag}(L), M_{1} \cap \operatorname{Inndiag}(L)\right)^{i}
$$

для $i \in\{1,2\}$. Кроме того, из $\left(\operatorname{Inndiag}(L), M_{1} \cap \operatorname{Inndiag}(L), M_{2} \cap \operatorname{Inndiag}(L)\right) \in \Pi$ и ранее рассмотренного случая $\left(^{*}\right)$ вытекает, что

$$
\left|M_{1} \cap M_{2} \cap L\right|=\left|\left(M_{1} \cap \operatorname{Inndiag}(L)\right) \cap\left(M_{2} \cap \operatorname{Inndiag}(L)\right) \cap L\right|=2^{1} 1 .
$$

На основании доказанного и в силу рассмотренного ранее случая $\left(^{*}\right)$ заключаем, что группы $\left(M_{1}, M_{2}\right)^{i}$ и $\left(M_{2}, M_{1}\right)^{i}$ для $i \in\{1,2\}$, а также группа $M_{1} \cap M_{2} \cap L$ имеют указанный в п. (b) вид.

Покажем, что произвольная тройка $\left(G, M_{1}^{*}, M_{2}^{*}\right)$ из П, где (далее мы используем введенные выше обозначения $\mathcal{V}, \mathcal{V}_{\left\{V_{1}, V_{2}, V_{3}, V_{4}\right\}}^{-}$и $\left.\mathcal{V}_{\left\{V_{1}, V_{2}, V_{3}, V_{4}\right\}}^{+}\right) \widehat{M_{i}^{*} \cap \operatorname{Inndiag}(L)}, i \in\{1,2\}$, есть стабилизатор в $\widehat{\operatorname{Inndiag}(L)}$ некоторого разложения $V=V_{i, 1}^{*}+V_{i, 2}^{*}+V_{i, 3}^{*}+V_{i, 4}^{*},\left\{V_{i, 1}^{*}, V_{i, 2}^{*}, V_{i, 3}^{*}, V_{i, 4}^{*}\right\} \in \mathcal{V}$, эквивалентна тройке $\left(G, M_{1}, M_{2}\right)$. Из $\left(G, M_{1}^{*}, M_{2}^{*}\right) \in \Pi$ следует (см. выше), что $\left(\operatorname{Inndiag}(L), M_{1}^{*} \cap\right.$ $\left.\operatorname{Inndiag}(L), M_{2}^{*} \cap \operatorname{Inndiag}(L)\right) \in$ П. Так как $M_{1}^{*}=N_{G}\left(M_{1}^{*} \cap \operatorname{Inndiag}(L)\right)$ и $\widehat{\operatorname{Inndiag}(L)}$ действует транзитивно на $\mathcal{V}$, то мы можем, не теряя общности (сопрягая в случае необходимости тройку $\left(G, M_{1}^{*}, M_{2}^{*}\right)$ подходящим элементом из Inndiag $\left.(L)\right)$, считать, что $M_{1}^{*}=M_{1}$ и $\left\{V_{1,1}^{*}, V_{1,2}^{*}, V_{1,3}^{*}, V_{1,4}^{*}\right\}=\left\{V_{1}, V_{2}, V_{3}, V_{4}\right\}$. Но тогда, как было доказано ранее, $\left(\operatorname{Inndiag}(L), M_{1}^{*} \cap\right.$ $\left.\operatorname{Inndiag}(L), M_{2}^{*} \cap \operatorname{Inndiag}(L)\right) \in \Pi$ влечет $\left\{V_{2,1}^{*}, V_{2,2}^{*}, V_{2,3}^{*}, V_{2,4}^{*}\right\} \in \mathcal{V}_{\left\{V_{1}, V_{2}, V_{3}, V_{4}\right\}}^{-} \cup \mathcal{V}_{\left\{V_{1}, V_{2}, V_{3}, V_{4}\right\}}^{+}$. Кроме того, ранее было доказано, что действие группы $\widehat{M_{1} \cap \operatorname{Inndiag(L)}}=\left\langle\tilde{h}, \widehat{M_{1} \cap P S O(V)}\right\rangle$ на $\mathcal{V}_{\left\{V_{1}, V_{2}, V_{3}, V_{4}\right\}}^{-} \cup \mathcal{V}_{\left\{V_{1}, V_{2}, V_{3}, V_{4}\right\}}^{+}$транзитивно. Поэтому некоторый элемент из $M_{1} \cap \operatorname{Inndiag}(L)$ сопряжением переводит $M_{2}^{*} \cap \operatorname{Inndiag}(L)$ в $M_{2} \cap \operatorname{Inndiag}(L)$, а следовательно, с учетом $M_{2}^{*}=N_{G}\left(M_{2}^{*} \cap \operatorname{Inndiag}(L)\right)$ и $M_{2}=N_{G}\left(M_{2} \cap \operatorname{Inndiag}(L)\right)$, также переводит $M_{2}^{*}$ в $M_{2}$. Таким образом, сопряжение тройки $\left(G, M_{1}^{*}=M_{1}, M_{2}^{*}\right)$ подходящим элементом из $M_{1} \cap \operatorname{Inndiag}(L)$ переводит ее в $\left(G, M_{1}, M_{2}\right)$, что доказывает эквивалентность $\left(G, M_{1}^{*}, M_{2}^{*}\right)$ и $\left(G, M_{1}, M_{2}\right)$.

Покажем, что в общем случае (как при $\varepsilon_{1}=+$ и $q=5$, так и при $\varepsilon_{1}=-$ и $q=3$, как при

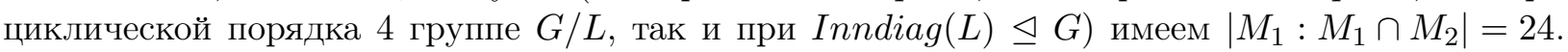
Для этого заметим прежде всего, что множество всех троек вида $\left(G, M_{1}, M_{2}^{*}\right)$ из П инвариантно относительно сопряжения элементами из $M_{1}$.

Если $G / L$ - циклическая группа порядка 4 , то (поскольку все такие подгруппы $G$ группы $A u t(L)$ сопряжены в группе $A u t(L))$, мы можем, не теряя общности, предполагать, что $G \leq P C G O(V)$. При этом предположении, как было доказано при рассмотрении случая $\left(^{*}\right)$, во-первых, тройки вида $\left(G, M_{1}, M_{2}^{*}\right)$ из П характеризуются тем, что $\widetilde{M_{2}^{*}}$ есть стабилизатор в $\tilde{G}$ разложения $V=U_{1}+U_{2}+U_{3}+U_{4}$ для $\left\{U_{1}, U_{2}, U_{3}, U_{4}\right\} \in \mathcal{V}_{\left\{V_{1}, V_{2}, V_{3}, V_{4}\right\}}^{-} \cup \mathcal{V}_{\left\{V_{1}, V_{2}, V_{3}, V_{4}\right\}}^{+}$, а во-вторых, группа $\widetilde{M}_{1}$ действует транзитивно на $\mathcal{V}_{\left\{V_{1}, V_{2}, V_{3}, V_{4}\right\}}^{-} \cup \mathcal{V}_{\left\{V_{1}, V_{2}, V_{3}, V_{4}\right\}}^{+}$. Итак, множество всех троек вида $\left(G, M_{1}, M_{2}^{*}\right)$ из П имеет мощность 24 , причем группа $M_{1}$ транзитивно действует на нем (сопряжением). Так как одним из элементов этого множества является тройка $\left(G, M_{1}, M_{2}\right)$, стабилизатор которой в $M_{1}$ (при указанном действии сопряжением) есть $M_{1} \cap N_{G}\left(M_{2}\right)=M_{1} \cap M_{2}$, то в случае, когда $G / L$ - циклическая группа порядка 4 , равенство $\left|M_{1}: M_{1} \cap M_{2}\right|=24$ справедливо.

Пусть $G / L$ не есть циклическая группа порядка 4 и, следовательно, $\operatorname{Inndiag}(L) \unlhd G$. Тогда (см. выше) тройки вида $\left(G, M_{1}, M_{2}^{*}\right)$ из П характеризуются тем, что $\left(\operatorname{Inndiag}(L), M_{1} \cap\right.$ $\left.\operatorname{Inndiag}(L), M_{2}^{*} \cap \operatorname{Inndiag}(L)\right) \in \Pi$. Но, как было доказано при рассмотрении случая $\left({ }^{*}\right)$,

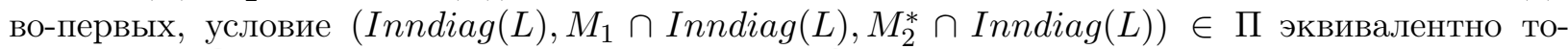
му, что $\widehat{M_{2}^{*} \cap \operatorname{Inndiag(L)}}$ есть стабилизатор в $\overline{\operatorname{Inndiag}(L)}$ разложения $V=U_{1}+U_{2}+U_{3}+U_{4}$ для $\left\{U_{1}, U_{2}, U_{3}, U_{4}\right\} \in \mathcal{V}_{\left\{V_{1}, V_{2}, V_{3}, V_{4}\right\}}^{-} \cup \mathcal{V}_{\left\{V_{1}, V_{2}, V_{3}, V_{4}\right\}}^{+}$, а во-вторых, группа $\widehat{M_{1} \cap \operatorname{Inndiag}(L)}$ действует транзитивно на $\mathcal{V}_{\left\{V_{1}, V_{2}, V_{3}, V_{4}\right\}}^{-} \cup \mathcal{V}_{\left\{V_{1}, V_{2}, V_{3}, V_{4}\right\}}^{+}$. Отсюда с учетом $\left(G, M_{1}, M_{2}^{*}\right)=$ 
$\left(N_{G}(\operatorname{Inndiag}(L)), N_{G}\left(M_{1} \cap \operatorname{Inndiag}(L)\right), N_{G}\left(M_{2}^{*} \cap \operatorname{Inndiag}(L)\right)\right)$ заключаем, что множество всех троек вида $\left(G, M_{1}, M_{2}^{*}\right)$ из П имеет мощность 24 , причем группа $M_{1}$ транзитивно действует на нем (сопряжением). Так как одним из элементов этого множества является тройка $\left(G, M_{1}, M_{2}\right)$, стабилизатор которой в $M_{1}$ (при указанном действии сопряжением) есть $M_{1} \cap N_{G}\left(M_{2}\right)=M_{1} \cap M_{2}$, то равенство $\left|M_{1}: M_{1} \cap M_{2}\right|=24$ справедливо и в случае, когда $G / L$ не есть циклическая группа порядка 4 .

Остается проверить последнее утверждение из п. (b) теоремы. Пусть $G / L$ изоморфна $A_{4}$ или $S_{4}$ и $d$ - элемент порядка 3 из $M_{1} \cap M_{2}$ (в силу $\left|M_{1}: M_{1} \cap M_{2}\right|=24$ и $\left|M_{1} \cap M_{2} \cap L\right|=2^{11}$ такой элемент существует, причем $d \notin L)$. Заметим, что $M_{1} \cap M_{2} \cap L \neq Q$, где $Q=O_{2}\left(M_{0}\right)$. Действительно, 2-группа $M_{1} \cap M_{2} \cap L$ не является силовской 2-подгруппой группы $M_{2} \cap L$ и, следовательно, нормализуется некоторым элементом из $\left(M_{2} \cap L\right) \backslash\left(M_{1} \cap M_{2} \cap L\right) \subseteq M_{2} \backslash M_{1}$, а $N_{G}(Q)=M_{1}$. Таким образом, $Q\left(M_{1} \cap M_{2} \cap L\right)-d$-инвариантная силовская 2-подгруппа в $M_{0}$, а следовательно, и в $L$.

Пусть $\tau^{*}-$ стандартный графовый автоморфизм порядка 3 группы $L$. Докажем, что $d$ сопряжен с $\tau^{*}$ относительно $A u t(L)$. Предположим противное. Тогда ввиду [13, 4.7.3, 4.9.2] $q=3$ и $d$ сопряжен с $\tau^{*} z$ относительно $A u t(L)$ для некоторого корневого элемента $z$ порядка 3 из $C_{L}\left(\tau^{*}\right)$, причем $C_{L}\left(\tau^{*} z\right)=C_{L}\left(\tau^{*}\right) \cap C_{L}(z)$ и $C_{L}\left(\tau^{*}\right) \cong G_{2}(3)$. Согласно [6] имеем $C_{L}(d) / O_{3}\left(C_{L}(d)\right) \cong S L_{2}(3)$. Поэтому силовская 2-подгруппа из $C_{L}(d)$ изоморфна группе кватернионов порядка 8. С другой стороны, как мы показали в предыдущем абзаце, элемент $d$ нормализует некоторую силовскую 2-подгруппу $S$ из $M_{0}$ и, следовательно, централизует подгруппу $Z(S)$ порядка 2 . Поскольку $Z(S)$ содержится в четверной подгруппе $K_{1}$, нормальной в $M_{1}$, а $d$ принадлежит $M_{1}$, то $d$ централизует четверную подгруппу $K_{1}$ из $S$. Полученное противоречие доказывает последнее утверждение из п. (b) теоремы.

Теорема доказана.

3 а м е ч а н и е. Группа $M_{1}$ из п. (а) теоремы содержится в [14, таблица I, строки 51-54]. Группа $M_{1}$ из п. (b) теоремы содержится в [14, таблица I, строка 55] при $q=5$ и содержится в [14, таблица I, строка 56] при $q=3$. В обоих пп. (а) и (b) теоремы с учетом [14] имеем $M_{1} \cap L<Y<X<L$, где $Y \cong 2^{6}: A_{8}$ и $X \cong \Omega_{8}^{+}(2)$. Из приведенного доказательства теоремы следует, что в п. (а) теоремы группа $M_{1} / O_{2}\left(M_{1}\right)$ действует точно и неприводимо на $O_{2}\left(M_{1}\right) / Z\left(O_{2}\left(M_{1}\right)\right)$ и неприводимо на $Z\left(O_{3}\left(M_{1}\right)\right)$. Можно доказать также, что в п. (b) теоремы группа $\left(M_{1} \cap L\right) / O_{2}\left(M_{1} \cap L\right)$ действует неприводимо на $Z\left(O_{2}\left(M_{1} \cap L\right)\right)$, тривиально на $\left[O_{2}\left(M_{1} \cap L\right), O_{2}\left(M_{1} \cap L\right)\right] / Z\left(O_{2}\left(M_{1} \cap L\right)\right)$ и вполне приводимо на $O_{2}\left(M_{1} \cap L\right) /\left[O_{2}\left(M_{1} \cap L\right), O_{2}\left(M_{1} \cap L\right)\right]$. Из приведенного доказательства теоремы следует, что любая тройка из п. (b) теоремы эквивалентна тройке $\left(G, M_{1}, M_{2}\right)$, у которой $O(V) \unlhd G \leq A u t(O(V))$ (причем либо $G / O(V)$ - циклическая группа порядка 4, либо Inndiag $(O(V)) \leq G), M_{1}=N_{G}\left(M_{1} \cap O(V)\right), M_{2}=N_{G}\left(M_{2} \cap O(V)\right), M_{1} \cap O(V)$ - стабилизатор в $O(V)$ ортогонального разложения $V=\left\langle e_{1}, f_{1}\right\rangle+\left\langle e_{2}, f_{2}\right\rangle+\left\langle e_{3}, f_{3}\right\rangle+\left\langle e_{4}, f_{4}\right\rangle$, где для каждого $1 \leq s \leq 4$ при $q=5$ имеют место равенства $\left(e_{s}, e_{s}\right)=0=\left(f_{s}, f_{s}\right)$ и $\left(e_{s}, f_{s}\right)=1$, а при $q=3$ имеют место равенства $\left(e_{s}, e_{s}\right)=1=\left(f_{s}, f_{s}\right)$ и $\left(e_{s}, f_{s}\right)=0, M_{2} \cap O(V)$ - стабилизатор в $O(V)$ ортогонального разложения $V=\left\langle e_{1}+f_{1}, e_{2}+f_{2}\right\rangle+\left\langle e_{1}-f_{1}, e_{2}-f_{2}\right\rangle+\left\langle e_{3}+f_{3}, e_{4}+f_{4}\right\rangle+\left\langle e_{3}-f_{3}, e_{4}-f_{4}\right\rangle$.

Авторы благодарны В. В. Кораблевой за помощь при вычислениях, использующих GAP.

Укажем на опечатки в [4, ч. III]:

вместо абзаца на с. 167, строки 1-3 снизу, и с. 168, строки 1-2 сверху, должно быть "Так как $C_{S}\left(S_{1}\right)=\left\langle z_{1}\right\rangle \times S_{2}=\left\langle z_{1}\right\rangle \times S_{1}^{u}$, имеем $E_{2} \cap Z(S)=\left\langle z_{2}\right\rangle$ и, следовательно, $E_{1}$ и $E_{2}$ - единственные четверные подгруппы из $O_{2}\left(M_{0}\right)$, все неединичные элементы которых сопряжены с $z_{1}$ в $L . " ;$

на с. 167 , строка 17 снизу, вместо $S \in S y l_{2}\left(M_{0}\right)$ должно быть $S=S_{1} \times S_{2} \in S y l_{2}\left(M_{0}\right)$;

на с. 168 , строка 18 снизу, вместо $\times$ должно быть $\oplus$;

на с. 169 , строка 9 сверху, вместо $S p_{2}(q)^{t}(q+1)^{t}: S_{t}$ должно быть $S p_{2}(q)^{t}: S_{t}$;

на с. 169, строка 22 сверху, вместо $P S L\left(V_{k}\right)$ должно быть $P S L^{\varepsilon}\left(V_{k}\right)$;

на с. 169 , строка 35 сверху, вместо $P S L\left(V_{k}\right)$ должно быть $P S L^{\varepsilon}\left(\tilde{g}\left(V_{1}\right)\right.$; 
на с. 170 , строка 13 снизу, вместо $O_{3}\left(M_{0}\right)$ должно быть $O_{2}\left(M_{0}\right)$;

на с. 170 , строка 7 снизу, вместо " $x_{1}=x^{2^{n-1}}, y_{1}=y^{2^{n-1}}, w_{1}=w^{2^{n-2}}$ " должно быть " $x_{1}=$ $x^{2^{k-1}}, y_{1}=y^{2^{k-1}}, w_{1}=w^{2^{k-3}}$ при $k>2 " ;$

на с. 170 , строка 6 снизу, вместо $O_{2}(M)$ должно быть $O_{2}\left(M_{0}\right)$;

на с. 171 , строка 4 сверху, вместо " $L_{1}$ не принадлежит $A$ " должно быть " $L_{2}$ не принадлежит $A$ ";

на с. 171, строка 11 сверху, вместо $w^{2}$ должно быть $w$;

на с. 171, строка 12 сверху, вместо "то $M_{0}$ централизует $w_{1}$ " должно быть "то $k>2$ и $M_{0}$ централизует $w_{1} "$.

\section{СПИСОК ЛИТЕРАТУРЫ}

1. Бурбаки Н. Группы и алгебры Ли. М.: Мир, 1972. 334 с.

2. Кондратьев А.С. Нормализаторы силовских 2-подгрупп в конечных простых группах // Матем. заметки. 2005. Т. 78, № 3. С. 368-376.

3. Кондратьев А.С., Трофимов В.И. Стабилизаторы вершин графов и усиленная версия гипотезы Симса // Докл. АН. 1999. Т. 364, № 6. С. 741-743.

4. Кондратьев А.С., Трофимов В.И. Стабилизаторы вершин графов с примитивными группами автоморфизмов и усиленная версия гипотезы Симса. I, II, III // Тр. Ин-та математики и механики УpO РAH. 2014. T. 20, № 4. C. 143-152; 2016. T. 22, № 2. C. 177-187; 2016. T. 22, № 4. C. 163-172.

5. Aschbacher M. On the maximal subgroups of the finite classical groups // Invent. Math. 1984. Vol. 76, no. 3. P. 469-514. doi: 10.1007/BF01388470.

6. Atlas of finite groups / J.H. Conway [et. al.]. Oxford: Clarendon Press, 1985. 252 p. ISBN: 0198531990.

7. Barry M.J.J. Large abelian subgroups of Chevalley groups // J. Austral. Math. Soc. Ser. A. 1979. Vol. 27, no. 1. P. 59-87. doi: 10.1080/00927879008823931.

8. Bray J.N., Holt D.F., Roney-Dougal C.M. The maximal subgroups of the low-dimensional finite classical groups. Cambridge: Cambridge University Press, 2013. 438 p. (London Math. Soc. Lect. Note Ser.; vol. 407).

9. Carter R.W. Simple groups of Lie type. London: Wiley, 1972. 331 p. ISBN: 0471137359.

10. The GAP Group, GAP - Groups, Algorithms, and Programming, Ver. 4.9.1. 2018. URL: http://www.gap-system.org.

11. Gerono G.C. Note sur la résolution en nombres entiers et positifs de l'équation $x^{m}=y^{n}+1 / /$ Nouv. Ann. Math. (2). 1870. Vol. 9. P. 469-471.

12. Gorenstein D. Finite groups. N. Y.: Harper and Row, 1968. 528 p.

13. Gorenstein D., Lyons R., Solomon R. The classification of the finite simple groups. Number 3. Part I. Providence, RI: Amer. Math. Soc. 1998. 420 p. (Math. Surveys Monogr.; vol. 40, no. 3).

14. Kleidman P.B. The maximal subgroups of the finite 8-dimensional orthogonal groups $P \Omega_{8}^{+} q$ ) and of their automorphism groups // J. Algebra. 1987. V. 110, no. 1. P. 173-242. doi: 10.1016/0021-8693(87)90042-1.

15. Kleidman P.B., Liebeck M.W. The subgroup structure of the finite classical groups. Cambridge: Cambridge University Press, 1990. 304 p. ISBN: 978-0-8218-0391-2 .

16. Timmesfeld F.G. Amalgams with rank 2 groups of Lie-type in characteristic 2. Preprint. Giessen: University Giessen, 1984. 126 p.

Кондратьев Анатолий Семенович

д-р физ.-мат. наук, профессор, зав. сектором

Институт математики и механики им. Н.Н. Красовского УрО РАН;

Уральский федеральный университет, г. Екатеринбург

e-mail: A.S.Kondratiev@imm.uran.ru

Трофимов Владимир Иванович

д-р физ.-мат. наук, ведущий науч. сотрудник

Институт математики и механики им. Н.Н. Красовского УрО РАН;

Уральский федеральный университет, г. Екатеринбург

e-mail: trofimov@imm.uran.ru 


\section{REFERENCES}

1. Bourbaki N. Groupes et algebres de Lie (Chapt. IV-VI). Paris: Hermann, 1968, 282 p. doi: 10.1007/9783-540-34491-9. Translated to Russian under the title Gruppy i algebry Li (glavy IV - VI). Moscow: Mir Publ., 1972, 334 p.

2. Kondrat'ev A.S. Normalizers of the Sylow 2-subgroups in finite simple groups. Math. Notes, 2005, vol. 78, no. 3-4, pp. 338-346. doi: 10.1007/s11006-005-0133-9 .

3. Kondrat'ev A.S., Trofimov V.I. Stabilizers of graph's vertices and a strengthened version of the Sims conjecture. Dokl. Math., 1999, vol. 59, no. 1, pp. 113-115.

4. Kondrat'ev A.S., Trofimov V.I. Stabilizers of vertices of graphs with primitive automorphism groups and a strong version of the Sims conjecture, I,II, III. Proc. Inst. Steklov Math., 2015, vol. 289, suppl. 1, pp. 146-155, doi: 10.1134/S0081543815050132; 2016, vol. 295, suppl. 1, pp. 89-100, doi: 10.1134/S0081543816090108; 2017, vol. 299, suppl. 1, pp. 113-122, doi: $10.1134 / \mathrm{S} 0081543817090140$.

5. Aschbacher M. On the maximal subgroups of the finite classical groups. Invent. Math., 1984, vol. 76, no. 3, pp. 469-514. doi: 10.1007/BF01388470.

6. Conway J.H., Curtis R.T., Norton S.P., Parker R.A., Wilson R.A. Atlas of finite groups. Oxford: Clarendon Press, 1985, 252 p. ISBN: 0198531990.

7. Barry M.J.J. Large abelian subgroups of Chevalley groups. J. Austral. Math. Soc., Ser. A, 1979, vol. 27, no. 1, pp. 59-87. doi: 10.1017/S1446788700016645.

8. Bray J.N., Holt D.F., Roney-Dougal C.M. The maximal subgroups of the low-dimensional finite classical groups. Cambridge: Cambridge Univ. Press, 2013, London Math. Soc. Lect. Note Ser., vol. 407, 438 p. doi: 10.1017/CBO9781139192576.

9. Carter R.W. Simple groups of Lie type. London: Wiley, 1972, 331 p. ISBN: 0471137359.

10. The GAP Group. GAP - Groups, Algorithms, and Programming, Ver. 4.9.1., 2018. Available on: https://www.gap-system.org .

11. Gerono G.C. Note sur la résolution en nombres entiers et positifs de l'équation $x^{m}=y^{n}+1$. Nouv. Ann. Math. (2), 1870, vol. 9, pp. 469-471.

12. Gorenstein D. Finite groups. N Y: Harper and Row, 1968, 528 p. ISBN (2nd ed.): 978-0821843420.

13. Gorenstein D., Lyons R., Solomon R. The classification of the finite simple groups. Providence: Amer. Math. Soc., 1998, Number 3, Part I, Ser. Math. Surveys Monogr., vol. 40, no. 3, 420 p.

ISBN: 978-0-8218-0391-2 .

14. Kleidman P.B. The maximal subgroups of the finite 8-dimensional orthogonal groups $\left.P \Omega_{8}^{+} q\right)$ and of their automorphism groups. J. Algebra, 1987, vol. 110, no. 1, pp. 173-242. doi: 10.1016/0021-8693(87)90042-1.

15. Kleidman P.B., Liebeck M.W. The subgroup structure of the finite classical groups. Cambridge: Cambridge Univ. Press, 1990, London Math. Soc. Lect. Note Ser., vol. 129, 304 p. ISBN: 0-521-35949-X.

16. Timmesfeld F.G. Amalgams with rank 2 groups of Lie-type in characteristic 2. Preprint. Giessen: University Giessen, 1984, 126 p.

The paper was received by the Editorial Office on December 25, 2017.

Funding Agency: This work was supported by the Russian Science Foundation (project no. 1411-00061-П).

Anatolii Semenovich Kondrat'ev, Dr. Phys.-Math. Sci., N.N. Krasovskii Institute of Mathematics and Mechanics, Ural Branch of the Russian Academy of Sciences, Yekaterinburg, 620990 Russia; Ural Federal University, Yekaterinburg, 620002 Russia, e-mail: A.S.Kondratiev@imm.uran.ru.

Vladimir Ivanovich Trofimov, Dr. Phys.-Math. Sci., N.N. Krasovskii Institute of Mathematics and Mechanics, Ural Branch of the Russian Academy of Sciences, Yekaterinburg, 620990 Russia; Ural Federal University, Yekaterinburg, 620002 Russia, trofimov@imm.uran.ru . 